\title{
A synthesis strategy yielding skeletally diverse small molecules combinatorially
}

\author{
Martin D. Burke, Eric M. Berger, and Stuart L. Schreiber \\ Contribution from the Broad Institute of Harvard and MIT, Department of Chemistry and Chemical Biology, \\ Howard Hughes Medical Institute, Harvard University, 12 Oxford Street, Cambridge, MA 02138.
}

Part A

\section{Supporting information}

I. General methods

II. Synthesis of model compounds

III. Building block testing

IV. Parallel synthesis

V. Encoded split-pool synthesis

Part B

VI. Spectra of model compounds

Part C

VII. Encoding and LCMS data from products of split-pool synthesis
$\mathrm{S} 1-\mathrm{S} 2$

S3-S22

S23-S28

S29-S34

S35-S50

S51-S69

S70-S190

\section{General methods}

Materials. Commercially available reagents were obtained from Aldrich Chemical Co. (Milwaukee, WI), Fluka Chemical Corp. (Milwaukee, WI), Bachem (Bubendorf, Switzerland), and MoscowMedChemLabs (Moscow, Russia) and used without further purification unless otherwise noted. All solvents were dispensed from a solvent purification system that passes solvents through packed columns (THF, $\mathrm{Et}_{2} \mathrm{O}, \mathrm{CH}_{3} \mathrm{CN}$, and $\mathrm{CH}_{2} \mathrm{Cl}_{2}$ : dry neutral alumina; hexane, benzene, and toluene: dry neutral alumina and Q5 reactant; DMF: activated molecular sieves). Water was double distilled. Triethylamine, diisopropylethylamine, and 2,6-lutidine were distilled under nitrogen from $\mathrm{CaH}_{2}$. Macrobeads were prepared by M. Narovlyansky at Harvard's ICCB: Longwood as previously described. ${ }^{1}$

Solution-phase reactions. All solution-phase reactions were performed in oven- or flame-dried glassware under positive argon pressure unless otherwise indicated. Reactions were monitored by analytical thin-layer chromatography performed using indicated solvent on E. Merck silica gel $60 \mathrm{~F}_{254}$ plates $(0.25 \mathrm{~mm})$. Compounds were visualized with a UV lamp $\left(\square_{254}\right)$ and/or staining with cerium ammonium molybdate.

\footnotetext{
${ }^{1}$ John A. Tallarico and coworkers. J. Comb. Chem. 2001, 3, 312.
} 
Solid-phase reactions. All solid-phase reactions were performed in oven- or flame-dried glassware (I-Chem vials or Wheaton vials, fitted with Teflon-coated caps) with gentle mixing provided by Thermoline Vari-Mix shaker or a Vortex Genie-2 vortexer (VWR 58815-178, setting V1-V2) fitted with a 60 microtube insert, unless otherwise indicated. After reactions were completed, resin was isolated by filtration in $10 \mathrm{ml}$ Amersham columns on a Vac-Man laboratory Vacuum Manifold (Promega A7231) fitted with nylon 3-way stopcocks (Biorad 7328107). Resin was then washed as indicated and solvent was removed under argon flow and/or in vacuo. All compounds were cleaved from macrobeads using the following standard procedure: To resin in a polypropylene eppendorf tube at $\mathrm{rt}$ under ambient was added a freshly prepared solution of $5 \% \mathrm{HF} / \mathrm{Pyr}$ in THF (10-100 $\square \mathrm{L}$ per $\mathrm{mg}$ of resin). The resulting mixture was then agitated at $\mathrm{rt}$ for $2 \mathrm{~h}$. The reaction was then quenched with the addition of neat methoxytrimethylsilane or ethoxytrimethylsilane $(2 / 1 \mathrm{v} / \mathrm{v}$ relative to $5 \% \mathrm{HF} / \mathrm{Pyr}$ in $\mathrm{THF}$ solution). The resulting mixture was then agitated at $\mathrm{rt}$ for 10 minutes, and the solution was then transferred to a Wheaton vial. Resin was washed twice with THF. The combined reaction solution and wash solutions were concentrated in vacuo and the cleaved product was then analyzed as indicated.

Purification and analysis. Flash chromatography was performed using the indicated solvent on E. Merck silica gel 60. All yields refer to compounds cleaved from 75-85 $\mathrm{mg}$ of macrobeads and purified by flash chromatography. Infrared spectra were recorded as a thin film on $\mathrm{NaCl}$ plates on a Nicolet 5PC FTIR spectrometer with internal referencing. Absorption maxima $\left(\square_{\max }\right)$ are reported in wavenumbers $\left(\mathrm{cm}^{-1}\right)$. ${ }^{1} \mathrm{H}$ NMR spectra were recorded at $23{ }^{\circ} \mathrm{C}$ on a Varian Unity/Inova500 (500 MHz) spectrometer. Chemical shifts ( $\square$ ) are reported in parts per million (ppm) downfield from tetramethylsilane and referenced to residual protium in the NMR solvent $\left(\mathrm{CDCl}_{3}, \square=7.26\right)$. Data are reported as follows: chemical shift, multiplicity $(\mathrm{s}=$ singlet, $\mathrm{d}=$ doublet, $\mathrm{t}=$ triplet, $\mathrm{q}=$ quartet, $\mathrm{m}=$ multiplet, $\mathrm{b}=$ broad $)$, coupling constant $(J)$ in Hertz $(\mathrm{Hz})$, integration, and assignment. ${ }^{13} \mathrm{C}$ NMR spectra were recorded at $23{ }^{\circ} \mathrm{C}$ on a Varian Unity/Inova400 (400 MHz) spectrometer. Chemical shifts ( $\square$ ) are reported in parts per million (ppm) downfield from tetramethylsilane and referenced to carbon resonances in the NMR solvent $\left(\mathrm{CDCl}_{3}, \square=77.0\right.$, center line). Nanotube solid-phase MAS ${ }^{1} \mathrm{H}$ NMR were obtained at 23 ${ }^{\circ} \mathrm{C}$ in $\mathrm{CD}_{2} \mathrm{Cl}_{2}$ on a Varian Inova 600 instrument fitted with a magic-angle spinning nanoprobe. Reverse-phase LCMS data was obtained with a Gilson/Finnigan LCMS system. LCMS chromatography was performed on a SymmetryShield ${ }^{\mathrm{TM}} \mathrm{RP}_{8}, 3.5 \mathrm{uM}, 4.6$ x 100mm column (Waters Corporation, Milford, MA, Batch \#111) using a flow rate of $1 \mathrm{ml} / \mathrm{min}$ and a $10 \mathrm{~min}$ gradient of $20-80 \% \mathrm{CH}_{3} \mathrm{CN}$ in water, constant $0.1 \%$ formic acid, with $\mathrm{UV}$ detection at 214 and $280 \mathrm{~nm}$. MS analysis was performed with a Finnigan Aqa MS detector with ES+ ionization. Chiral LC was performed on a Gilson HPLC system using a Chiralpak ${ }^{\circledR}$ AS $^{\mathrm{TN}} 250 \times 4.6 \mathrm{~mm}$ column (Amylose tris-[(S)- $\square$-methylbenzyl carbamate] coated on $10 \square \mathrm{m}$ silica-gel substrate, Chiral Technologies Inc., Exton, PA) using an eluent of $4 \%$ IPA in hexanes and a flow rate of 1 $\mathrm{ml} / \mathrm{min}$. High resolution mass spectra (HRMS) were obtained at the mass spectrometry facility at Harvard University. 


\section{Synthesis of model compounds}

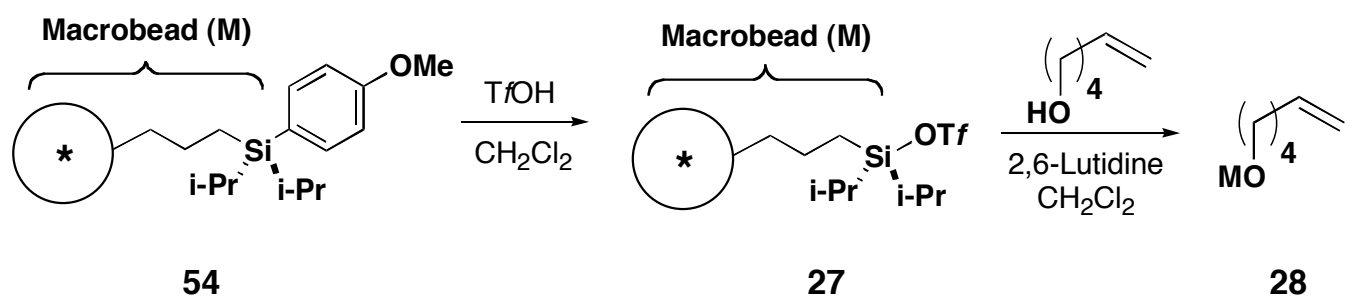

\section{Macrobead-bound-5-hexen-1-ol (28)}

3-[Diisopropyl( $p$-methoxyphenyl)silyl]propyl functionalized "macrobeads" (400 mg, estimated loading $\sim 1.3 \mathrm{meq} \mathrm{Si} / \mathrm{g}, \sim 0.52 \mathrm{mmol}$ ) in a $20 \mathrm{~mL}$ polypropylene tube at $\mathrm{rt}$ under argon were allowed to swell in $\mathrm{CH}_{2} \mathrm{Cl}_{2}(15 \mathrm{ml})$ for $10 \mathrm{~min}$. The colorless beads were then filtered and again washed with $\mathrm{CH}_{2} \mathrm{Cl}_{2}(15 \mathrm{~mL} \times 10 \mathrm{~min}$.), and then resuspended in a $2.5 \%(\mathrm{v} / \mathrm{v})$ solution of TMSCl in $\mathrm{CH}_{2} \mathrm{Cl}_{2}(15 \mathrm{~mL})$ for $30 \mathrm{~min}$. The beads were again filtered and washed thrice with $\mathrm{CH}_{2} \mathrm{Cl}_{2}$ (5 min each) and then suspended in a $3 \%(\mathrm{v} / \mathrm{v})$ solution of trifluoromethanesulfonic acid in $\mathrm{CH}_{2} \mathrm{Cl}_{2}(9.2 \mathrm{~mL}, 3.12 \mathrm{mmol})$ for $20 \mathrm{~min}$ during which the reaction tube was shaken periodically and the beads turned orange. After filtration, the orange-colored beads were again thrice washed with $\mathrm{CH}_{2} \mathrm{Cl}_{2}$ and then resuspended in a minimum volume of $\mathrm{CH}_{2} \mathrm{Cl}_{2}(1 \mathrm{~mL})$. Freshly distilled 2,6-lutidine was then added (485 uL, $4.2 \mathrm{mmol})$ resulting in bead discoloration followed by 5 -hexen-1-ol $(500 \mathrm{uL}, 4.2 \mathrm{mmol})$. The resulting colorless reaction mixture was then shaken manually and let stand at rt for $12 \mathrm{~h}$. The beads were then filtered, washed with $\mathrm{CH}_{2} \mathrm{Cl}_{2}$ ( 5 x $15 \mathrm{~mL}$ x $5 \mathrm{~min}$. each), and the solvent was removed under argon flow followed by residual solvent removal in vacuo to yield colorless resin $28(372 \mathrm{mg})$ loaded with 5-hexen-1-ol. MAS ${ }^{1} \mathrm{H}$ NMR (600 MHz, $\mathrm{CD}_{2} \mathrm{Cl}_{2}$, selected resonances) $\square 5.81$ (br s), 5.00 (br d, $J=17.0 \mathrm{~Hz}$ ), 4.93 (br $\mathrm{d}, J=8 \mathrm{~Hz}$ ), 3.65 (br s).

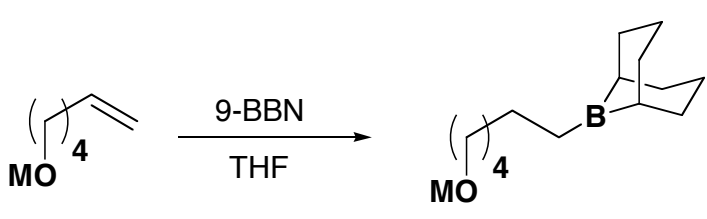

28
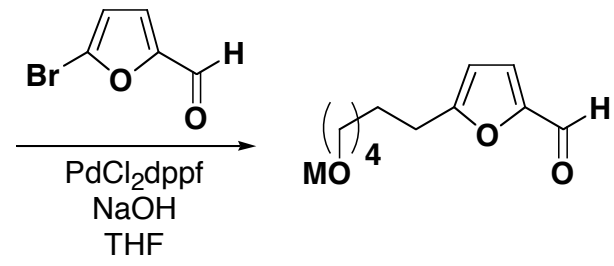

30

\section{Macrobead-bound-5-(6-hydroxyhexyl)-furan-2-carbaldehyde (30)}

Colorless beads 28 (500 mg, max theoretical loading $1.3 \mathrm{meq} . / \mathrm{g}, 0.65 \mathrm{mmol}$ ) were washed with THF ( $2 \times 10 \mathrm{~mL} \times 10 \mathrm{~min}$ each) at $\mathrm{rt}$ and then resuspended in $15 \mathrm{~mL}$ THF. A $0.5 \mathrm{M}$ solution of 9 -BBN in THF $(10 \mathrm{~mL}, 5.0 \mathrm{mmol})$ was then added and the resulting colorless reaction mixture was manually agitated and let stand at $\mathrm{rt}$ for $5 \mathrm{~h}$. The reaction solution was then removed via cannula and the colorless resin was washed thoroughly with THF $(5 \times 15 \mathrm{~mL}$ x 10 min each). To the resin was then added solid $\mathrm{PdCl}_{2} \mathrm{dppf}(6.1 \mathrm{mg}, 0.0075 \mathrm{mmol}$ ) (adding increased amounts of catalyst led to compound cleavage from macrobeads), 5-bromo-2furaldehyde (438 mg, $2.5 \mathrm{mmol})$ via cannula as a solution in THF $(6.25 \mathrm{~mL})$, and a $1 \mathrm{M}$ aq. solution of $\mathrm{NaOH}(1.25 \mathrm{~mL}, 1.25 \mathrm{mmol})$. The resulting orange reaction mixture was sealed under a cloud of argon and heated at $65{ }^{\circ} \mathrm{C}$ with periodic manual agitation for $18 \mathrm{~h}$ (reaction 
mixture turned dark brown). The yellow/orange resin was then isolated by filtration and washed as follows, $4 \times\left(5 \times \mathrm{THF}, 5 \times \mathrm{H}_{2} \mathrm{O}, 5 \times \mathrm{THF}\right.$, THF: $\left.\mathrm{H}_{2} \mathrm{O} 3: 1 \times 30 \mathrm{~min}\right), 5 \times \mathrm{THF}$, THF $\times 30 \mathrm{~min}, 5$ x $\mathrm{CH}_{2} \mathrm{Cl}_{2}, \mathrm{CH}_{2} \mathrm{Cl}_{2} \times 30 \mathrm{~min}, 5 \mathrm{x}$ anh. $\mathrm{CH}_{2} \mathrm{Cl}_{2}$, anh. $\mathrm{CH}_{2} \mathrm{Cl}_{2} \times 30 \mathrm{~min}$, and then the solvent was removed in vacuo to yield $535 \mathrm{mg}$ of yellow/orange product resin 30. $5 \mathrm{mg}$ of this resin was treated with HF/Pyridine cleavage conditions (see General Methods) to yield the crude alcohol $\mathbf{3 0}_{\mathrm{M}=\mathrm{H}}$ with LCMS purity $>85 \%\left(\square_{214}, \mathrm{t}_{\mathrm{R}} 4.82 \mathrm{~min}\right)$. $75 \mathrm{mg}$ of the product resin 30 was treated with HF/Pyridine cleavage conditions and the crude product was purified by flash chromatography $\left(\mathrm{SiO}_{2}\right.$, hexanes:EtOAc 1:2) to afford alcohol $\mathbf{3 0}_{\mathbf{M}=\mathbf{H}}$ as a yellow oil $(10.0 \mathrm{mg}$, 0.679 meq./g, $58 \%$ over two steps based on estimated maximum of meq. Si/g). The macrobeadbound product 30 was stored for more than one year at $4{ }^{\circ} \mathrm{C}$ (sealed in a glass jar along with anhydrous calcium sulfate) without appreciable decomposition. In contrast, once cleaved from the solid-support, alcohol $\mathbf{3 0}_{\mathrm{M}=\mathrm{H}}$ readily decomposed upon prolonged exposure to ambient $(>12$ h). TLC (hexanes:EtOAc 1:2) $\mathrm{R}_{\mathrm{f}}=0.27$; FTIR (film, $\mathrm{cm}^{-1}$ ) 3426, 2932, 2859, 1674, 1518, 1399 , 1024; ${ }^{1} \mathrm{H}$ NMR $\left(500 \mathrm{MHz}, \mathrm{CDCl}_{3}\right.$ ) $\square 9.51$ (s, 1H, CHO), 7.17 (d, J=3.5 Hz, 1H, C 3 -H), 6.23 (d, $\left.J=4.0 \mathrm{~Hz}, 1 \mathrm{H}, \mathrm{C}_{4}-\mathbf{H}\right), 3.64\left(\mathrm{t}, J=7.0 \mathrm{~Hz}, 2 \mathrm{H}, \mathrm{CH}_{2} \mathrm{OH}\right), 2.73(\mathrm{t}, J=7.5 \mathrm{~Hz}, 2 \mathrm{H}$, FurCH$), 1.72$ (m, 2H, FurCH $\mathrm{CH}_{2}$ ), 1.57 (m, 2H, $\left.\mathrm{CH}_{2} \mathrm{CH}_{2} \mathrm{OH}\right), 1.39$ (m, 4H, $\mathrm{CH}_{2} \mathrm{CH}_{2} \mathrm{CH}_{2} \mathrm{CH}_{2} \mathrm{OH}$ ); ${ }^{13} \mathrm{C}$ NMR $\left(100 \mathrm{MHz}, \mathrm{CDCl}_{3}\right) \square 176.9,163.9,151.8,123.6,108.7,62.8,32.5,28.9,28.3,27.5,25.3$; HRMS $\left(\mathrm{ES}^{+}\right)$calculated for $\mathrm{C}_{11} \mathrm{H}_{16} \mathrm{O}_{3}(\mathrm{M}+\mathrm{H})^{+}:$197.1177, found: 197.1177.
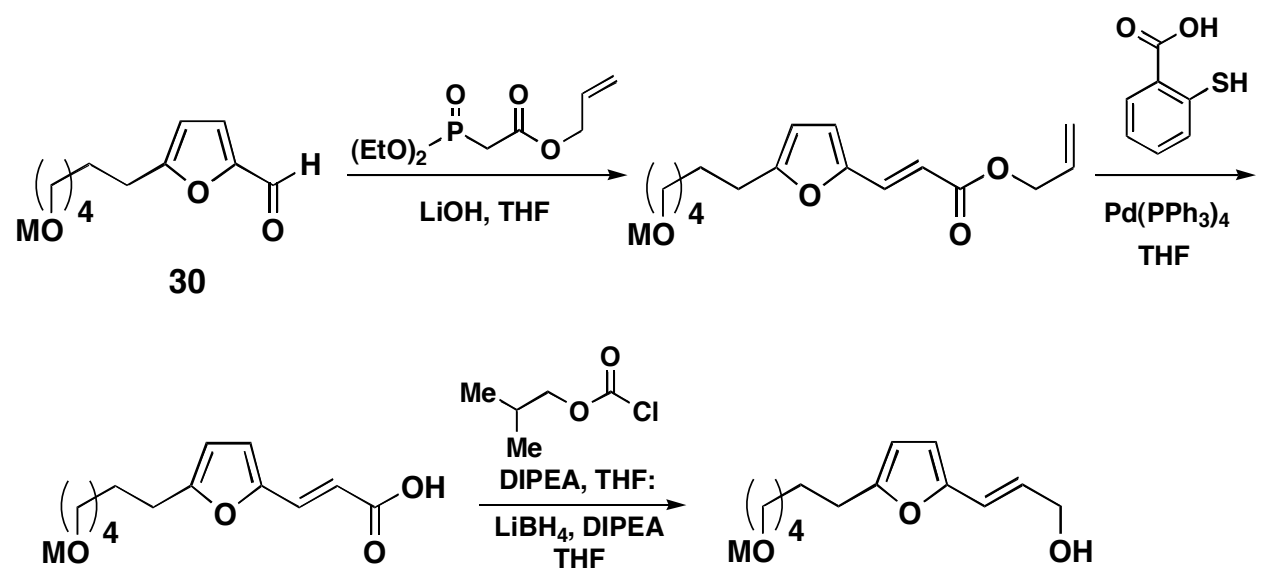

31

\section{Macrobead-bound-trans-3-[5-(6-hydroxy-hexyl)-furan-2-yl]-prop-2-en-1-ol (31)}

To a stirred solution of allyldiethylphosphonoacetate $(0.664 \mathrm{~mL}, 3.15 \mathrm{mmol})$ in THF $(10.5 \mathrm{~mL})$ at $\mathrm{rt}$ was added solid $\mathrm{LiOH}(151 \mathrm{mg}$. $6.29 \mathrm{mmol})$. The resulting colorless mixture was stirred vigorously at $\mathrm{rt}$ for $4 \mathrm{~h}$. The stir bar was then removed and resin 30 (315 $\mathrm{mg}, 0.679$ $\mathrm{meq} / \mathrm{g}, 0.214 \mathrm{mmol}$ ) was added. The resulting reaction mixture was sealed under a cloud of argon and tumbled at $\mathrm{rt}$ for $25 \mathrm{~h}$. The resin was then isolated by filtration and washed as follows: 5 x THF, $5 \times \mathrm{H}_{2} \mathrm{O}, 5 \times$ THF, THF:dilute aq. $\mathrm{NH}_{4} \mathrm{Cl}$ (sat. aq. $\mathrm{NH}_{4} \mathrm{Cl}: \mathrm{H}_{2} \mathrm{O}$ 1:2) 1:1 x 1 h. 5 x THF, $5 \times \mathrm{H}_{2} \mathrm{O}, 5$ x THF, THF: $\mathrm{H}_{2} \mathrm{O} 3: 1 \times 1 \mathrm{~h}, 5$ x THF, THF $\times 30 \mathrm{~min}, 5 \times \mathrm{CH}_{2} \mathrm{Cl}_{2}, \mathrm{CH}_{2} \mathrm{Cl}_{2}$ x $20 \mathrm{~min}, 5$ x anhydrous $\mathrm{CH}_{2} \mathrm{Cl}_{2}$, anhydrous $\mathrm{CH}_{2} \mathrm{Cl}_{2} \times 10 \mathrm{~min}$. Solvent was then removed in vacuo to yield $336 \mathrm{mg}$ of yellow-orange resin. This resin $(331 \mathrm{mg})$ was then added to a yellow mixture of $\mathrm{Pd}\left(\mathrm{PPh}_{3}\right)_{4}(382 \mathrm{mg}, 0.331 \mathrm{mmol})$ in THF $(7.2 \mathrm{~mL})$. To this mixture was then added solid thiosalicylic acid $(510 \mathrm{mg}, 3.31 \mathrm{mmol})$, and the resulting dark red mixture was sealed under a cloud of argon, covered with aluminum foil, and tumbled at $\mathrm{rt}$ for $24 \mathrm{~h}$. Resin was then isolated by filtration and washed as follows: $5 \times(5 \times \mathrm{THF}$, THF $\times 1 \mathrm{~h}), 5 \times \mathrm{CH}_{2} \mathrm{Cl}_{2}, \mathrm{CH}_{2} \mathrm{Cl}_{2} \times 20 \mathrm{~min}, 5 \mathrm{x}$ 
anhydrous $\mathrm{CH}_{2} \mathrm{Cl}_{2}$, anhydrous $\mathrm{CH}_{2} \mathrm{Cl}_{2} \times 10 \mathrm{~min}$. Solvent was then removed in vacuo to yield 322 mg of yellow-orange resin. This resin $(317 \mathrm{mg})$ was then washed twice with anhydrous THF and then resuspended in THF $(29 \mathrm{~mL})$. Diisopropylethylamine $(2.75 \mathrm{~mL}, 15.8 \mathrm{mmol})$ was then added and the resulting mixture was cooled to $0{ }^{\circ} \mathrm{C}$. To this mixture was added 4methylmorpholine $(0.035 \mathrm{~mL}, 0.317 \mathrm{mmol})$ and isobutylchloroformate $(0.411 \mathrm{~mL}, 3.17 \mathrm{mmol})$. The resulting mixture was maintained at $0{ }^{\circ} \mathrm{C}$ for $2 \mathrm{~h}$, with periodic manual agitation every 30 minutes. The reaction solution was removed via cannula and the resin was washed with $8.6 \%$ (v/v) diisopropylethylamine in THF $\left(3 \times 15 \mathrm{~mL} \times 5 \mathrm{~min}\right.$ each) at $0{ }^{\circ} \mathrm{C}$. Resin was then resuspended in a solution of $8.6 \%(\mathrm{v} / \mathrm{v})$ diisopropylethylamine in THF $(40 \mathrm{~mL})$ at $0{ }^{\circ} \mathrm{C}$, and to this mixture was added solid $\mathrm{LiBH}_{4}(21 \mathrm{mg}, 0.95 \mathrm{mmol})$. The resulting mixture was sealed under a cloud of argon and tumbled at $4{ }^{\circ} \mathrm{C}$ for $24 \mathrm{~h}$. The resin was then isolated by filtration at $\mathrm{rt}$ and washed as follows: 5 x THF, $5 \times \mathrm{H}_{2} \mathrm{O}, 5 \mathrm{x}$ THF, THF:dilute aq. $\mathrm{NH}_{4} \mathrm{Cl}$ (sat. aq. $\mathrm{NH}_{4} \mathrm{Cl}: \mathrm{H}_{2} \mathrm{O}$ 1:2) 1:1 x 1 h. $5 \times$ THF, $5 \times \mathrm{H}_{2} \mathrm{O}, 5$ x THF, THF: $\mathrm{H}_{2} \mathrm{O} 3 / 1 \times 1 \mathrm{~h}, 5 \times \mathrm{THF}, 5 \times \mathrm{H}_{2} \mathrm{O}, 5 \times \mathrm{THF}$, THF $\times 1$ h, $5 \times \mathrm{CH}_{2} \mathrm{Cl}_{2}, \mathrm{CH}_{2} \mathrm{Cl}_{2} \times 30 \mathrm{~min}, 5$ x anhydrous $\mathrm{CH}_{2} \mathrm{Cl}_{2}$, anhydrous $\mathrm{CH}_{2} \mathrm{Cl}_{2} \times 30$ min and then solvent was removed in vacuo to yield light yellow product resin 31 (320 mg). $5 \mathrm{mg}$ of this product resin was then treated with $\mathrm{HF} /$ Pyridine cleavage conditions (see General Methods) to yield the crude diol $\mathbf{3 1}_{\mathrm{M}=\mathrm{H}}$ with LCMS purity $68 \%\left(\square_{214}, \mathrm{t}_{\mathrm{R}} 5.80 \mathrm{~min}\right) .75 \mathrm{mg}$ of this resin was then treated with HF/Pyridine cleavage conditions and the crude product was purified by flash chromatography $\left(\mathrm{SiO}_{2}\right.$, hexanes:EtOAc 1:2) to afford diol $\mathbf{3 1}_{\mathbf{M = H}}$ a colorless solid [8.1 mg, 0.482 meq./g, Theoretical yield 0.637 meq./g, 76\% from 30, E:Z >20:1 ( ${ }^{1} \mathrm{H}$ NMR)]. TLC (hexanes:EtOAc 1:2) $\mathrm{R}_{\mathrm{f}}=0.24$; FTIR (film, $\mathrm{cm}^{-1}$ ) 3349, 2928, 2857, 1661, 1588, 1532, 1463, 1380, 1254; ${ }^{1} \mathrm{H}$ NMR $\left(500 \mathrm{MHz}, \mathrm{CDCl}_{3}\right) \square 6.37(\mathrm{dd}, J=16 \mathrm{~Hz}, 1 \mathrm{~Hz}, 1 \mathrm{H}, \mathrm{FurCH}=\mathrm{CH}), 6.21$ (dt, $J=16,6 \mathrm{~Hz}, 1 \mathrm{H}$, FurCH=CH), $6.13(\mathrm{~d}, J=3.5 \mathrm{~Hz}, 1 \mathrm{H}$, FurH), 5.95 (d, $J=3.5 \mathrm{~Hz}, 1 \mathrm{H}$, FurH), $4.27\left(\mathrm{~d}, J=5.5 \mathrm{~Hz}, 2 \mathrm{H}, \mathrm{CHCH}_{2} \mathrm{OH}\right), 3.64\left(\mathrm{t}, J=7 \mathrm{~Hz}, 2 \mathrm{H}, \mathrm{CH}_{2} \mathrm{CH}_{2} \mathrm{OH}\right) 2.61(\mathrm{t}, J=7.5 \mathrm{~Hz}, 2 \mathrm{H}$, FurCH $\left.H_{2}\right), 1.66\left(\mathrm{~m}, 2 \mathrm{H}, \quad\right.$ FurCH $\left.\mathrm{CH}_{2}\right), 1.58\left(\mathrm{~m}, 2 \mathrm{H}, \mathrm{CH}_{2} \mathrm{CH}_{2} \mathrm{OH}\right), 1.39(\mathrm{~m}, 4 \mathrm{H}$, $\left.\mathrm{CH}_{2} \mathrm{CH}_{2} \mathrm{CH}_{2} \mathrm{CH}_{2} \mathrm{OH}\right) ;{ }^{13} \mathrm{C}$ NMR $\left(100 \mathrm{MHz}, \mathrm{CDCl}_{3}\right) \square 156.4,150.6,125.5,119.8,109.1,106.6$, 63.5, 62.9, 32.6, 28.9, 28.0, 27.9, 25.4; HRMS (ES ${ }^{+}$) calculated for $\mathrm{C}_{13} \mathrm{H}_{20} \mathrm{O}_{3}(\mathrm{M}-\mathrm{H})^{-}$: 223.1334, found: 223.1333 .
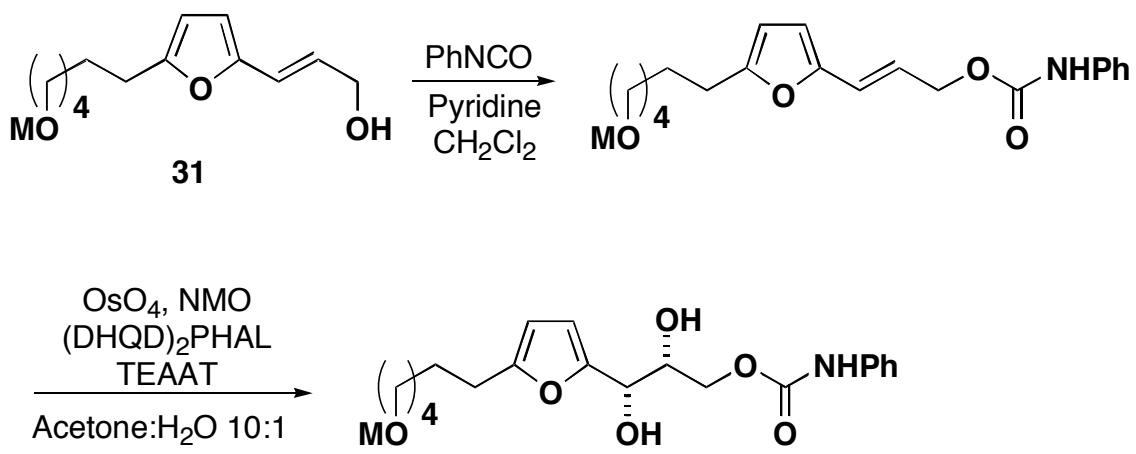

32

\section{Macrobead-bound-(2R,3S)-phenyl-carbamic acid 2,3-dihydroxy-3-[5-(6-hydroxy-hexyl)- furan-2-yl]-propyl ester (32)}

Light yellow beads 31 (220 mg, 0.482 meq./g, 0.106 mmol) were washed with $\mathrm{CH}_{2} \mathrm{Cl}_{2}(2$ x $10 \mathrm{~mL}$ x $10 \mathrm{~min}$ each) at $\mathrm{rt}$ and then resuspended in $\mathrm{CH}_{2} \mathrm{Cl}_{2}(11 \mathrm{~mL})$. To this mixture at $\mathrm{rt}$ was added pyridine $(0.356 \mathrm{~mL}, 4.41 \mathrm{mmol})$ and phenyl isocyanate $(0.239 \mathrm{~mL}, 2.20 \mathrm{mmol})$. The 
resulting mixture was sealed under a cloud of argon and tumbled at $\mathrm{rt}$ for $24 \mathrm{~h}$. Resin was then isolated by filtration and washed as follows: 5 x THF, $5 \times \mathrm{H}_{2} \mathrm{O}, 5 \mathrm{x}$ THF, THF:dil. aq. $\mathrm{NaHCO}_{3}$ (sat. aq. $\mathrm{NaHCO}_{3}: \mathrm{H}_{2} \mathrm{O}$ 1:2) 1:1 x 1 h, 5 x THF, $5 \times \mathrm{H}_{2} \mathrm{O}, 5 \times \mathrm{THF}$, THF:dil. aq. $\mathrm{NH}_{4} \mathrm{Cl}$ (sat. aq. $\mathrm{NH}_{4} \mathrm{Cl}: \mathrm{H}_{2} \mathrm{O}$ 1:2) 1:1 x $1 \mathrm{~h}, 5$ x THF, 5 x $\mathrm{H}_{2} \mathrm{O}, 5$ x THF, THF: $\mathrm{H}_{2} \mathrm{O}$ 3:1 x 1 h, 5 x THF, THF x 1 h, $5 \times \mathrm{CH}_{2} \mathrm{Cl}_{2}, \mathrm{CH}_{2} \mathrm{Cl}_{2} \times 30 \mathrm{~min}$, and then residual solvent was removed in vacuo to yield $245 \mathrm{mg}$ of yellow resin. A separate vessel was then charged with (DHQD) 2 PHAL (10.6 mg, 0.0135 $\mathrm{mmol})$, tetraethylammonium acetate tetrahydrate $(113 \mathrm{mg}, 0.433 \mathrm{mmol})$ and 4-methylmorpholine $N$-oxide (76.2 $\mathrm{mg}, 0.650 \mathrm{mmol}$ ). This solid mixture was dissolved in a solution of acetone:water 10:1 at $\mathrm{rt}$ under ambient and to this clear, colorless solution was added $\mathrm{OsO}_{4}$ as a $2.5 \mathrm{wt} \%$ solution in tert-butyl alcohol $(0.060 \mathrm{ml}, 0.00542 \mathrm{mmol})$. The resulting clear, yellow-tinted solution was let stand at $\mathrm{rt}$ with periodic manual agitation for $15 \mathrm{~min}$ and then cooled to $0{ }^{\circ} \mathrm{C}$. The resin $(217 \mathrm{mg})$ was then added and the resulting mixture was sealed under ambient and tumbled at $4{ }^{\circ} \mathrm{C}$ for $48 \mathrm{~h}$. The reaction solution was then removed via syringe and quenched with excess sodium metabisulfite, and the resin was washed with acetone:water 10:1 (1 x $5 \mathrm{~mL}$ × 10 min, $1 \times 15 \mathrm{~mL} \times 30 \mathrm{~min})$ at $4{ }^{\circ} \mathrm{C}$, and then isolated by filtration and washed as follows: $5 \times$ THF, $10 \%$ pyridine in THF x $1 \mathrm{~h}, 5 \times \mathrm{THF}, 10 \%$ pyridine in THF x $12 \mathrm{~h}, 5 \mathrm{x}$ THF, $10 \%$ pyridine in THF x 4 h, 5 x THF, $10 \%$ pyridine in THF x 4 h, $5 \times$ THF, $5 \times \mathrm{H}_{2} \mathrm{O}, 5 \times$ THF, THF:dilute aq. $\mathrm{NaHCO}_{3}$ (sat. aq. $\mathrm{NaHCO}_{3}: \mathrm{H}_{2} \mathrm{O}$ 1:2) 1:1 x $45 \mathrm{~min}, 5$ x THF, 5 x H $\mathrm{NH}_{4} \mathrm{Cl}$ (sat. aq. $\mathrm{NH}_{4} \mathrm{Cl}: \mathrm{H}_{2} \mathrm{O}$ 1:2) 1:1 x $45 \mathrm{~min}, 5$ x THF, 5 x $\mathrm{H}_{2} \mathrm{O}, 5$ x THF, THF: $\mathrm{H}_{2} \mathrm{O} 3: 1 \times 1 \mathrm{~h}$, 5 x THF, THF x 45 min, 5 x DMF, DMF x 45 min, 5 x THF, THF x 45 min, 5 x anh. THF, anh. THF x $30 \mathrm{~min}$, and then solvent was removed under argon flow followed by residual solvent removal in vacuo. $5.2 \mathrm{mg}$ of this resin was then treated with $\mathrm{HF} /$ Pyridine cleavage conditions (see General Methods) to yield crude triol 32 $\mathbf{M}_{\mathbf{M}=\mathbf{H}}$ with LCMS purity $>90 \%\left(\square_{214}, \mathrm{t}_{\mathrm{R}} 5.92 \mathrm{~min}\right)$. $75.2 \mathrm{mg}$ of this resin was then treated with $\mathrm{HF} /$ Pyridine cleavage conditions and the crude product was purified by flash chromatography $\left(\mathrm{SiO}_{2}\right.$, EtOAc:MeOH 100:0 $\rightarrow$ 90:10) to afford

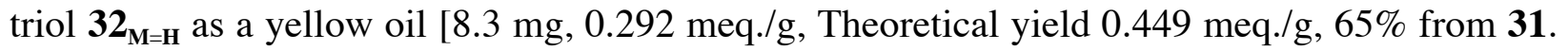
The enantioselectivity obtained in this reaction $(e e=66 \%)$ was determined after converting 32 $\rightarrow 35$ (vide infra). ${ }^{2}$ TLC (EtOAc:MeOH 99:1) $\mathrm{R}_{\mathrm{f}}=0.33$; FTIR (film, $\mathrm{cm}^{-1}$ ) 3322, 2932, 2858, 1711, 1601, 1547, 1501, 1445, 1315, 1224, 1055; ${ }^{1} \mathrm{H}$ NMR $\left(500 \mathrm{MHz}, \mathrm{CDCl}_{3}\right) \square 7.38-7.28(\mathrm{~m}$, 4H, PhH), 7.07 (t, J= 7 Hz, 1H, PhH), 6.96 (br s, 1H, NH), 6.27 (d, J = 3 Hz, 1H, FurH), 5.94 $(\mathrm{d}, J=3 \mathrm{~Hz}, 1 \mathrm{H}, \operatorname{FurH}), 4.65(\mathrm{~d}, J=5.5 \mathrm{~Hz}, 1 \mathrm{H}, \mathrm{FurCHOH}), 4.27(\mathrm{dd}, J=11.5,3.5 \mathrm{~Hz}, 1 \mathrm{H}$, $\left.\mathrm{NCO}_{2} \mathrm{CHH}\right), 4.22-4.13\left(\mathrm{~m}, 2 \mathrm{H}, \mathrm{NCO}_{2} \mathrm{CHH}\right.$ and $\left.\mathrm{NCO}_{2} \mathrm{CHHCHOH}\right), 3.62(\mathrm{t}, J=6.5 \mathrm{~Hz}, 2 \mathrm{H}$, $\mathrm{CH}_{2} \mathrm{OH}$ ), 3.26 (br s, 1H, OH), 2.97 (br s, 1H, OH), 2.60 (t, $J=7 \mathrm{~Hz}, 2 \mathrm{H}$, FurCH$), 1.63$ (m, 2H, $\left.\operatorname{FurCH}_{2} \mathrm{CH}_{2}\right), 1.55\left(\mathrm{~m}, 2 \mathrm{H}, \mathrm{CH}_{2} \mathrm{CH}_{2} \mathrm{OH}\right), 1.35\left(\mathrm{~m}, 4 \mathrm{H}, \mathrm{CH}_{2} \mathrm{CH}_{2} \mathrm{CH}_{2} \mathrm{CH}_{2} \mathrm{OH}\right) ;{ }^{13} \mathrm{C}$ NMR $(100$ $\left.\mathrm{MHz}, \mathrm{CDCl}_{3}\right) \square 156.7,153.7,150.8,137.5,129.1,123.7,118.7,108.9$, 105.7, 72.0, 68.0, 66.0, 62.8, 32.4, 28.7, 27.8, 27.7, 25.3; HRMS $\left(\mathrm{ES}^{+}\right)$calculated for $\mathrm{C}_{20} \mathrm{H}_{27} \mathrm{NO}_{6}(\mathrm{M}+\mathrm{Na})^{+}:$400.1736, found: 400.1737 .

\footnotetext{
${ }^{2}$ In a solution-phase study, the dihydroxylation of a related substrate proceeded with $>90 \%$ ee. It is not clear why the enantioselectivity was diminished for the solid-phase reaction.
} 


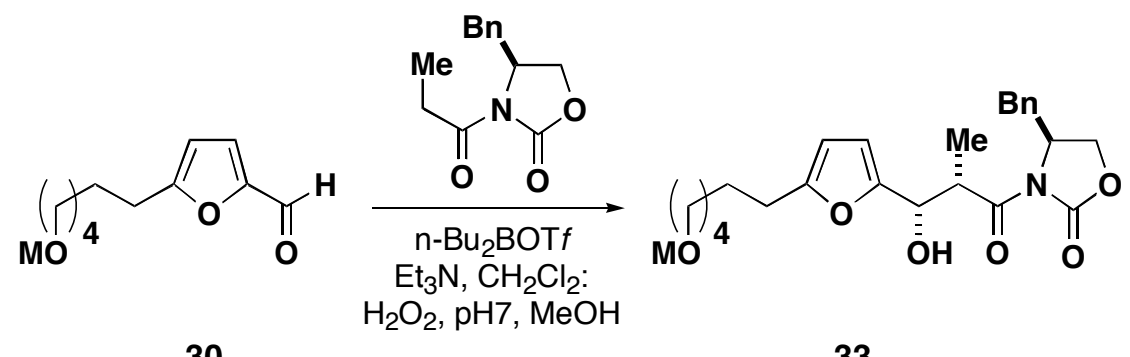

30

33

Macrobead-bound-(4S)-4-benzyl-3-\{(3S,2S)-3-hydroxy-3-[5-(6-hydroxy-hexyl)-furan-2-yl]2-methyl-propionyl\}-oxazolidin-2-one (33)

Yellow-orange beads 30 (365 mg, 0.679 meq./g, $0.248 \mathrm{mmol}$ ) were washed with $\mathrm{CH}_{2} \mathrm{Cl}_{2}$ $\left(2 \times 15 \mathrm{~mL} \times 10 \mathrm{~min}\right.$ each) at $\mathrm{rt}$, and then cooled to $-78{ }^{\circ} \mathrm{C}$. In a separate vessel, to a stirred solution of $(S)$-(+)-4-benzyl-3-propionyl-2-oxazolidinone (426 mg, $1.83 \mathrm{mmol})$ in $\mathrm{CH}_{2} \mathrm{Cl}_{2}(7.3$ $\mathrm{mL}$ ) at $0{ }^{\circ} \mathrm{C}$ was added a $1 \mathrm{M}$ solution of dibutylboron triflate in $\mathrm{CH}_{2} \mathrm{Cl}_{2}(1.92 \mathrm{~mL}, 1.92 \mathrm{mmol}$, $n B u_{2} B O T f$ solution was obtained from Aldrich chemical company and stored at $-26^{\circ} \mathrm{C}$ upon delivery. Best results were obtained when this reagent was used within 2 weeks of shipping date) followed by triethylamine $(0.305 \mathrm{~mL}, 2.19 \mathrm{mmol})$. The resulting colorless (or slightly yellowtinted) enolate solution was cooled to $-78{ }^{\circ} \mathrm{C}$ and then transferred rapidly via cannula to the vessel containing 30. The resulting mixture was sealed under a cloud of argon and maintained at $-78{ }^{\circ} \mathrm{C}$ for $48 \mathrm{~h},-26{ }^{\circ} \mathrm{C}$ for $24 \mathrm{~h}$, and $0{ }^{\circ} \mathrm{C}$ for $1 \mathrm{~h}$ (with periodic manual agitation about once every $8 \mathrm{~h}$ ). The reaction was then quenched with the addition of $\mathrm{pH} 7$ phosphate buffer $(7 \mathrm{~mL})$, $\mathrm{MeOH}(7 \mathrm{~mL})$, and $30 \%$ aq. $\mathrm{H}_{2} \mathrm{O}_{2}(4.7 \mathrm{~mL})$, and the resulting mixture was tumbled at $4{ }^{\circ} \mathrm{C}$ for 12 h. Resin was then isolated by filtration and washed as follows: $5 \times \mathrm{CH}_{2} \mathrm{Cl}_{2}, 5 \times \mathrm{DMF}, 5 \times \mathrm{THF}, 5$ x $\mathrm{CH}_{2} \mathrm{Cl}_{2}, \mathrm{CH}_{2} \mathrm{Cl}_{2}$ x $1 \mathrm{~h}, 5$ x DMF, DMF x $1 \mathrm{~h}, 5$ x THF, THF x $1 \mathrm{~h}, 5$ x $\mathrm{CH}_{2} \mathrm{Cl}_{2}, \mathrm{CH}_{2} \mathrm{Cl}_{2}$ x 30 min, $5 \times$ anhydrous $\mathrm{CH}_{2} \mathrm{Cl}_{2}$, anhydrous $\mathrm{CH}_{2} \mathrm{Cl}_{2} \times 30 \mathrm{~min}$, and residual solvent was removed in vacuo to yield 33 as light yellow beads $(431 \mathrm{mg}) .5 .2 \mathrm{mg}$ of this resin was then treated with $\mathrm{HF} /$ Pyridine cleavage conditions (see General Methods) to yield crude diol $\mathbf{3 3}_{\mathbf{M}=\mathbf{H}}$ with LCMS purity $>90 \%\left(\square_{214}, \mathrm{t}_{\mathrm{R}} 7.74 \mathrm{~min}\right)$ and d.r. $>20: 1\left({ }^{1} \mathrm{H} \mathrm{NMR}\right)$. $75.2 \mathrm{mg}$ of this resin was then treated with HF/Pyridine cleavage conditions and the crude product was purified by flash chromatography $\left(\mathrm{SiO}_{2}\right.$, hexanes:EtOAc $\left.1: 1 \rightarrow 1: 2\right)$ to afford diol $\mathbf{3 3}_{\mathrm{M}=\mathrm{H}}$ a yellow oil $(18.2 \mathrm{mg}$, 0.0424 mmol, 0.563 meq./g, Theoretical yield 0.586 meq./g, $96 \%$ from 30). TLC (hexanes:EtOAc 1:2) $\mathrm{R}_{\mathrm{f}}=0.30$; FTIR (film, $\mathrm{cm}^{-1}$ ) 3442, 2933, 2859, 1781, 1696, 1454, 1387, 1210, 1108, 1012; ${ }^{1} \mathrm{H}$ NMR (500 MHz, $\mathrm{CDCl}_{3}$ ) $\square$ 7.35-7.27 (m, 3H, PhH), 7.19 (app d, $J=6.5$ $\mathrm{Hz}, 2 \mathrm{H}, \mathrm{PhH}), 6.17$ (d, $J=3 \mathrm{~Hz}, 1 \mathrm{H}, \operatorname{FurH}), 5.90(\mathrm{~d}, J=3 \mathrm{~Hz}, 1 \mathrm{H}$, FurH), 5.01 (m, 1H, FurCHOH), $4.62\left(\mathrm{~m}, 1 \mathrm{H}, \mathrm{C}_{4}-\mathbf{H}\right), 4.16\left(\mathrm{~m}, 3 \mathrm{H}, \mathrm{COCHCH}_{3}\right.$ and $\left.\mathrm{C}_{5}-\mathbf{H H}\right), 3.62(\mathrm{t}, J=6 \mathrm{~Hz}, 2 \mathrm{H}$, $\left.\mathrm{CH}_{2} \mathrm{OH}\right), 3.24\left(\mathrm{dd}, J=13,3 \mathrm{~Hz}, 1 \mathrm{H}, \mathrm{C}_{4}-\mathrm{CHHPh}\right), 2.99$ (br d, $J=4 \mathrm{~Hz}, 1 \mathrm{H}$, FurCHOH), 2.78 (dd, $\left.J=13,9 \mathrm{~Hz}, 1 \mathrm{H}, \mathrm{C}_{4}-\mathrm{CHHPh}\right), 2.58\left(\mathrm{t}, J=7.5 \mathrm{~Hz}, 2 \mathrm{H}, \mathrm{FurCH}_{2}\right), 1.62\left(\mathrm{~m}, 2 \mathrm{H}, \mathrm{FurCH}_{2} \mathrm{CH}_{2}\right.$ ), $1.56\left(\mathrm{~m}, 2 \mathrm{H}, \mathrm{CH}_{2} \mathrm{CH}_{2} \mathrm{OH}\right), 1.36\left(\mathrm{~d}, J=6.5 \mathrm{~Hz}, 3 \mathrm{H}, \mathrm{CH}_{3}\right), 1.36\left(\mathrm{~m}, 4 \mathrm{H}, \mathrm{CH}_{2} \mathrm{CH}_{2} \mathrm{CH}_{2} \mathrm{CH}_{2} \mathrm{OH}\right)$; ${ }^{13} \mathrm{C}$ NMR $\left(100 \mathrm{MHz}, \mathrm{CDCl}_{3}\right) \square 176.2,156.0,152.8,152.1,135.0,129.4,128.9,127.4,107.3$, 105.3, 68.7, 66.2, 62.8, 55.2, 42.5, 37.8, 32.5, 28.7, 27.8 (2 carbons), 25.3, 12.2; HRMS (ES $\left.{ }^{+}\right)$ calculated for $\mathrm{C}_{24} \mathrm{H}_{31} \mathrm{NO}_{6}\left(\mathrm{M}+\mathrm{NH}_{4}\right)^{+}: 447.2495$, found: 447.2497 . 


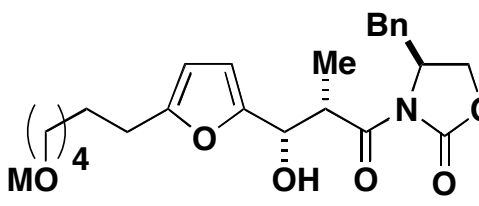

33

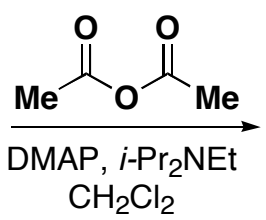

$\mathrm{CH}_{2} \mathrm{Cl}_{2}$<smiles>CC(=O)OCCc1ccc(C(C)C(=O)N2C(=O)OCC2Cc2ccccc2)o1</smiles>

34

Macrobead-bound-(4S)-4-benzyl-3-\{(3S,2S)-3-acetoxy-3-[5-(6-hydroxy-hexyl)-furan-2-yl]2-methyl-propionyl\}-oxazolidin-2-one (34).

Light yellow beads 33 ( $0.180 \mathrm{~g}, 0.563$ meq./g, $0.101 \mathrm{mmol})$ were washed with $\mathrm{CH}_{2} \mathrm{Cl}_{2}(2$ x $9 \mathrm{~mL}$ x $5 \mathrm{~min}$ each) at $\mathrm{rt}$ and then resuspended in $9 \mathrm{~mL} \mathrm{CH}_{2} \mathrm{Cl}_{2}$. To this mixture at rt was added diisopropylethylamine $(0.627 \mathrm{~mL}, 3.6 \mathrm{mmol})$, DMAP $(22 \mathrm{mg}, 0.18 \mathrm{mmol})$, and acetic anhydride $(0.170 \mathrm{~mL}, 1.8 \mathrm{mmol})$. The resulting mixture was sealed under a cloud of argon and tumbled at $\mathrm{rt}$ for $28 \mathrm{~h}$. Resin was then isolated by filtration and washed as follows: $5 \times \mathrm{CH}_{2} \mathrm{Cl}_{2}, 5$ x THF, $5 \times \mathrm{CH}_{2} \mathrm{Cl}_{2}, \mathrm{CH}_{2} \mathrm{Cl}_{2}$ x $45 \mathrm{~min}, 5 \mathrm{x} \mathrm{THF}$, THF x $45 \mathrm{~min}, 5 \times \mathrm{CH}_{2} \mathrm{Cl}_{2}, \mathrm{CH}_{2} \mathrm{Cl}_{2}$ x $45 \mathrm{~min}, 5 \mathrm{x}$ anh. $\mathrm{CH}_{2} \mathrm{Cl}_{2}$, anh. $\mathrm{CH}_{2} \mathrm{Cl}_{2} \times 20 \mathrm{~min}$. Solvent was then removed in vacuo to yield $\mathbf{3 4}$ as light yellow beads. $5.0 \mathrm{mg}$ of this resin was then treated with $\mathrm{HF} /$ Pyridine cleavage conditions (see General Methods) to yield crude alcohol $34_{\mathrm{M}=\mathrm{H}}$ with LCMS purity $>90 \%\left(\square_{214}, \mathrm{t}_{\mathrm{R}} 8.83 \mathrm{~min}\right) .75 .2$ mg of this resin was then treated with $\mathrm{HF} /$ Pyridine cleavage conditions and the crude product was purified by flash chromatography $\left(\mathrm{SiO}_{2}\right.$, hexanes:EtOAc $\left.1: 1 \rightarrow 1: 2\right)$ to afford alcohol $\mathbf{3 4}_{\mathrm{M}=\mathbf{H}}$ as a yellow oil [17.1 mg, $0.0363 \mathrm{mmol}, 0.482 \mathrm{meq} . / \mathrm{g}$, Theoretical yield $0.550 \mathrm{meq} . / \mathrm{g}, 88 \%$ from 33). TLC (hexanes:EtOAc 1:1) $\mathrm{R}_{\mathrm{f}}=0.17$; FTIR (film, $\mathrm{cm}^{-1}$ ) 3545, 2933, 2859, 1782, 1744, 1700, 1455, 1387, 1225, 1108, 1016; ${ }^{1} \mathrm{H}$ NMR (500 MHz, $\mathrm{CDCl}_{3}$ ) $\square$ 7.34-7.25 (m, 3H, PhH), $7.18(\mathrm{~d}, J=7 \mathrm{~Hz}, 2 \mathrm{H}, \mathrm{PhH}), 6.22$ (d, $J=3.5 \mathrm{~Hz}, 1 \mathrm{H}$, FurH), 6.14 (d, $J=7 \mathrm{~Hz}, 1 \mathrm{H}$, FurCHOAc), $5.89(\mathrm{~d}, J=3 \mathrm{~Hz}, 1 \mathrm{H}, \mathrm{FurH}), 4.51\left(\mathrm{~m}, 2 \mathrm{H}, \mathrm{C}_{4}-\mathbf{H}\right.$ and $\left.\mathrm{COCHCH}_{3}\right), 4.13\left(\mathrm{~m}, 2 \mathrm{H}, \mathrm{C}_{5}-\mathbf{H H}\right), 3.62(\mathrm{t}$, $\left.J=6 \mathrm{~Hz}, 2 \mathrm{H}, \mathrm{CH}_{2} \mathrm{OH}\right), 3.23\left(\mathrm{dd}, J=13,3 \mathrm{~Hz}, 1 \mathrm{H}, \mathrm{C}_{4}-\mathrm{CHHPh}\right), 2.75(\mathrm{dd}, J=13,9.5 \mathrm{~Hz}, 1 \mathrm{H}$, $\left.\mathrm{C}_{4}-\mathrm{CHHPh}\right), 2.56\left(\mathrm{t}, J=7.5 \mathrm{~Hz}, 2 \mathrm{H}, \mathrm{FurCH}_{2}\right), 2.09\left(\mathrm{~s}, 3 \mathrm{H}, \mathrm{COCH}_{3}\right), 1.60\left(\mathrm{~m}, 2 \mathrm{H}, \mathrm{FurCH}_{2} \mathrm{CH}_{2}\right)$, $1.56\left(\mathrm{~m}, 2 \mathrm{H}, \mathrm{CH}_{2} \mathrm{CH}_{2} \mathrm{OH}\right), 1.39-1.30\left(\mathrm{~m}, 4 \mathrm{H}, \mathrm{CH}_{2} \mathrm{CH}_{2} \mathrm{CH}_{2} \mathrm{CH}_{2} \mathrm{OH}\right), 1.33(\mathrm{~d}, J=7 \mathrm{~Hz}, 3 \mathrm{H}$, $\left.\mathrm{COCHCH}_{3}\right) ;{ }^{13} \mathrm{C}$ NMR $\left(100 \mathrm{MHz}, \mathrm{CDCl}_{3}\right)$ 173.4, 170.1, 156.6, 153.1, 149.1, 135.1, 129.4, 128.9, 127.4, 109.6, 105.5, 69.2, 66.2, 62.8, 55.4, 40.8, 37.8, 32.5, 28.8, 27.8, 27.7, 25.3, 21.0, 13.3; HRMS (ES ${ }^{+}$) calculated for $\mathrm{C}_{26} \mathrm{H}_{33} \mathrm{NO}_{7}(\mathrm{M}+\mathrm{Na})^{+}: 494.2155$, found: 494.2169 .

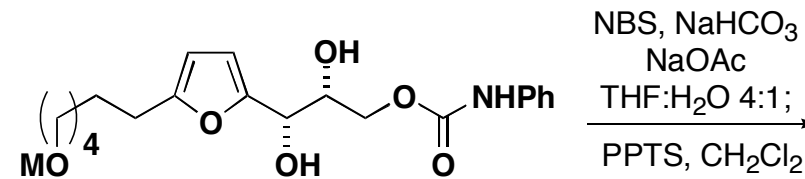

32

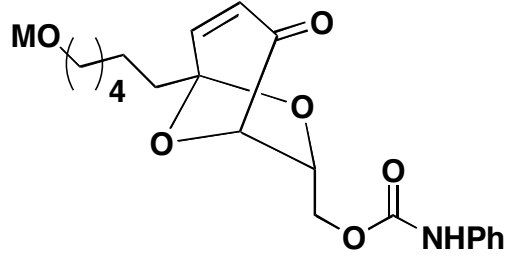

35

Macrobead-bound-phenyl-carbamic acid $(1 S, 5 S, 7 R)-5-(6-h y d r o x y-h e x y l)-2-0 x 0-6,8-$ dioxabi-cyclo[3.2.1]-oct-3-en-7-ylmethyl ester (35).

To a mixture of light yellow beads $32(0.090 \mathrm{~g}, 0.292 \mathrm{meq} . / \mathrm{g}, 0.026 \mathrm{mmol})$ in THF:water 4:1 at rt under ambient was added $\mathrm{NaHCO}_{3}(227 \mathrm{mg}, 2.70 \mathrm{mmol}), \mathrm{NaOAc}(111 \mathrm{mg}, 1.35 \mathrm{mmol})$, and $N$-bromosuccinimide $(160 \mathrm{mg}, 0.90 \mathrm{mmol})$. The resulting mixture was sealed, wrapped in 
foil, and tumbled at $\mathrm{rt}$ for $1 \mathrm{~h}$. Resin was then isolated by filtration and washed as follows: $5 \mathrm{x}$ THF, $5 \times \mathrm{H}_{2} \mathrm{O}, 5 \times \mathrm{THF}$, THF:water 3:1 x $1 \mathrm{~h}, 5 \times \mathrm{THF}$, THF x $1 \mathrm{~h}, 5 \times \mathrm{CH}_{2} \mathrm{Cl}_{2}, \mathrm{CH}_{2} \mathrm{Cl}_{2} \times 30$ min, $5 \mathrm{x}$ anh. $\mathrm{CH}_{2} \mathrm{Cl}_{2}$, anh. $\mathrm{CH}_{2} \mathrm{Cl}_{2} \times 30 \mathrm{~min}$, and solvent was then removed in vacuo. This resin was then transferred to a separate vessel containing a $0.00075 \mathrm{M}$ solution of pyridinium $p$ toluenesulfonate in $\mathrm{CH}_{2} \mathrm{Cl}_{2}(20 \mathrm{~mL})$. The resulting mixture was sealed under a cloud of argon and maintained at $40-45^{\circ} \mathrm{C}$ (oil bath) for $20 \mathrm{~h}$. Resin was then isolated by filtration and washed as follows: $5 \times$ THF, $5 \times \mathrm{H}_{2} \mathrm{O}, 5 \mathrm{x}$ THF, THF:dilute aq. $\mathrm{NaHCO}_{3}$ (sat. aq. $\mathrm{NaHCO}_{3}: \mathrm{H}_{2} \mathrm{O}$ 1:2) 1:1 x 1 h, 5 x THF, 5 x $\mathrm{H}_{2} \mathrm{O}, 5$ x THF, THF:dilute aq. $\mathrm{NH}_{4} \mathrm{Cl}$ (sat. aq. $\mathrm{NH}_{4} \mathrm{Cl}_{2} \mathrm{H}_{2} \mathrm{O}$ 1:2) 1:1 x $1 \mathrm{~h}, 5 \mathrm{x}$ THF, $5 \times \mathrm{H}_{2} \mathrm{O}, 5 \times \mathrm{THF}$, THF: $\mathrm{H}_{2} \mathrm{O} 3: 1 \times 1 \mathrm{~h}, 5$ x THF, THF x $1 \mathrm{~h}, 5 \times \mathrm{CH}_{2} \mathrm{Cl}_{2}, \mathrm{CH}_{2} \mathrm{Cl}_{2} \times 30$ min, $5 \mathrm{x}$ anh. $\mathrm{CH}_{2} \mathrm{Cl}_{2}$, anh. $\mathrm{CH}_{2} \mathrm{Cl}_{2} \times 30 \mathrm{~min}$. Solvent was then removed in vacuo to yield 133 as light yellow beads. $5.4 \mathrm{mg}$ of this resin was then treated with $\mathrm{HF} /$ Pyridine cleavage conditions (see General Methods) to yield crude alcohol $\mathbf{3 5}_{\mathrm{M}=\mathrm{H}}$ with LCMS purity $64 \%$, $\left(\square_{214}, \mathrm{t}_{\mathrm{R}} 7.04 \mathrm{~min}\right.$ ) (an impurity at $\mathrm{t}_{\mathrm{R}} 8.20$ min which was ${ }^{1} \mathrm{H}$ NMR-silent and had an MS isotope pattern consistent with an osmium-containing substance was not included in the purity calculation for this product; for additional purity information, see Catalog of spectra for ${ }^{1} \mathrm{H}$ NMR of crude and purified product $\mathbf{3 5}_{\mathrm{M}=\mathrm{H}}$ ). $80.8 \mathrm{mg}$ of the product resin was then treated with HF/Pyridine cleavage conditions and the crude product was purified by flash chromatography $\left(\mathrm{SiO}_{2}\right.$, hexanes:EtOAc 1:1 $\left.\rightarrow 1: 2\right)$ to afford alcohol $\mathbf{3 5}_{\mathrm{M}=\mathrm{H}}$ as a yellow oil [ $2.9 \mathrm{mg}, 0.00773 \mathrm{mmol}, 0.0 .096 \mathrm{meq} . \mathrm{g}$, Theoretical yield 0.0.292 meq./g, 33\% from 32). TLC (hexanes:EtOAc 1:2) $\mathrm{R}_{\mathrm{f}}=0.31$; FTIR (film, $\mathrm{cm}^{-1}$ ) 3323, 2932, 2859, 1705, 1599, 1537, 1491, 1445, 1400, 1309, 1220, 1075; ${ }^{1} \mathrm{H}$ NMR (500 MHz, $\left.\mathrm{CDCl}_{3}\right)$ 77.44-7.26 (m, 4H, PhH), $7.08(\mathrm{t}, J=7 \mathrm{~Hz}, 1 \mathrm{H}, \mathrm{PhH}), 7.02\left(\mathrm{~d}, J=10 \mathrm{~Hz}, 1 \mathrm{H}, \mathrm{C}_{4}-\mathbf{H}\right), 6.82(\mathrm{br}$, $1 \mathrm{H}, \mathrm{NH}), 6.08\left(\mathrm{dd}, J=10,1 \mathrm{~Hz}, 1 \mathrm{H}, \mathrm{C}_{3}-\mathbf{H}\right), 4.59\left(\operatorname{app~d}, J=6.5 \mathrm{~Hz}, 1 \mathrm{H}, \mathrm{C}_{1}-\mathbf{H}\right), 4.30-4.26(\mathrm{~m}$, $\left.2 \mathrm{H}, \mathrm{C}_{7}-\mathrm{CHH}\right), 4.06\left(\operatorname{app~q}, J=5 \mathrm{~Hz}, 1 \mathrm{H}, \mathrm{C}_{7}-\mathbf{H}\right), 3.64\left(\mathrm{t}, J=6 \mathrm{~Hz}, 2 \mathrm{H}, \mathrm{CH}_{2} \mathrm{OH}\right), 2.02-1.91$ (m, $\left.2 \mathrm{H}, \quad \mathrm{C}_{5}-\mathrm{CH}_{2}\right), \quad 1.60-1.48\left(\mathrm{~m}, \quad 4 \mathrm{H}, \quad \mathrm{C}_{5}-\mathrm{CH}_{2} \mathrm{CH}_{2} \mathrm{CH}_{2} \mathrm{CH}_{2} \mathrm{CH}_{2}\right), \quad 1.44-1.35 \quad\left(\mathrm{~m}, 4 \mathrm{H}, \mathrm{C}_{5^{-}}\right.$ $\left.\mathrm{CH}_{2} \mathrm{CH}_{2} \mathrm{CH}_{2} \mathrm{CH}_{2}\right) ;{ }^{13} \mathrm{C}$ NMR (100 MHz, $\mathrm{CDCl}_{3}$ ) $\square$ 194.0, 150.7 (2C), 132.1, 129.1, 126.5, 123.7, 120.2, 106.0, 94.4, 82.3, 73.2, 62.8, 34.5, 32.4, 29.1, 25.4, 22.3; HRMS $\left(\mathrm{ES}^{+}\right)$calculated for $\mathrm{C}_{20} \mathrm{H}_{25} \mathrm{NO}_{6}(\mathrm{M}+\mathrm{Na})^{+}: 398.1580$, found: 398.1570 .

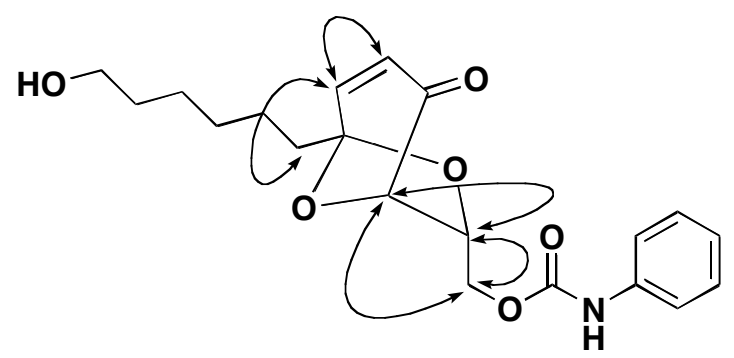

Selected nuclear Overhauser enhancements observed by two-dimensional NOESY experiment

Determination of enantiomeric excess: To determine the enantioselectivity achieved in the asymmetric dihydroxylation reaction used to generate 32, this reaction was repeated using the pseudoenantiomeric ligand (DHQ) 2 PHAL, and the resulting enantiomeric diol was subjected to the same oxidative and acidic conditions described above to yield the enantiomeric bicyclic ketal ent-35. A $\sim 1 / 1$ mixture of the two purified, enantiomeric bicyclic ketals $\left(\mathbf{3 5}_{\mathrm{M}=\mathrm{H}}\right.$ and $\left.\boldsymbol{e n t}-\mathbf{3 5}_{\mathrm{M}=\mathrm{H}}\right)$ was then prepared, and separation was achieved on a Chiralpak ${ }^{\circledR} \mathrm{AS}^{\mathrm{TM}} 250 \times 4.6 \mathrm{~mm}$ column (Amylose tris-[(S)- $\square$-methylbenzyl carbamate] coated on $10 \square \mathrm{m}$ silica-gel substrate, Chiral Technologies Inc., Exton, PA) using a flow rate of $1 \mathrm{ml} / \mathrm{min}$ and an eluent of 4\% IPA in hexanes 
$\left(\mathrm{t}_{\mathrm{R}} \mathbf{3 5}_{\mathrm{M}=\mathrm{H}}=3.13 \mathrm{~min}, \mathrm{t}_{\mathrm{R}}\right.$ ent $\left.\mathbf{- 3 5} 5_{\mathrm{M}=\mathrm{H}}=4.09 \mathrm{~min}\right)$. Using this LC method, the enantiomeric excess achieved in the dihydroxylation reaction was determined to be $66 \%$; the stereochemistry of the major isomer was assigned using the Sharpless mnemonic.

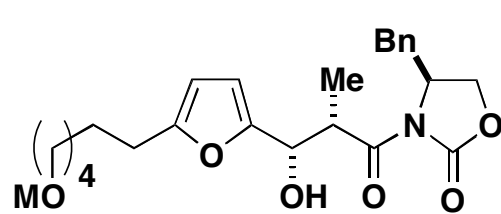

33

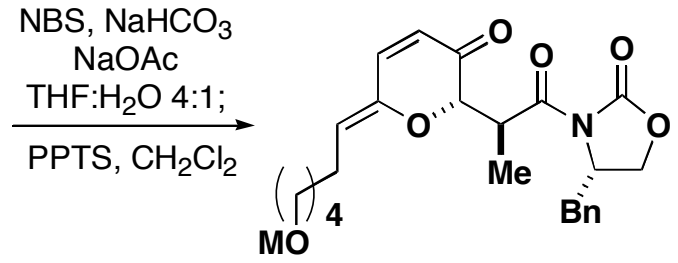

36

Macrobead-bound-(4S)-4-benzyl-3-\{((2S)-2-[(2S)-6-(6-hydroxy-hexylidene)-3-oxo-3,6dihydro-2H-pyran-2-yl]-propionyl $\}$-oxazolidin-2-one (36)

Light yellow beads $33(0.090 \mathrm{~g}, 0.563 \mathrm{meq} . / \mathrm{g}, 0.051 \mathrm{mmol})$ were treated with the same reaction conditions and washing protocols described above for the transformation of $\mathbf{3 2} \rightarrow \mathbf{3 5}$. Solvent was then removed in vacuo to yield $\mathbf{3 6}$ as light yellow beads. $5.2 \mathrm{mg}$ of this resin was then treated with HF/Pyridine cleavage conditions (see General Methods) to yield crude alcohol 36 $_{\mathrm{M}=\mathrm{H}}$ with LCMS purity $86 \%\left(\square_{214}, \mathrm{t}_{\mathrm{R}} 8.15 \mathrm{~min}\right)$. $84.6 \mathrm{mg}$ of this resin was then treated with $\mathrm{HF} / \mathrm{Pyridine}$ cleavage conditions and the crude product was purified by flash chromatography $\left(\mathrm{SiO}_{2}\right.$, hexanes:EtOAc 1:1 $\rightarrow$ 1:2) to afford alcohol $\mathbf{3 6}_{\mathrm{M}=\mathrm{H}}$ as a yellow oil [7.2 $\mathrm{mg}, 0.0168 \mathrm{mmol}$, 0.199 meq./g, Theoretical yield 0.564 meq./g, 35\% from 33. (The yield for this reaction, as determined by ${ }^{1} \mathrm{H}$ NMR using an internal standard, was much higher when carried out on small scale, i.e., 1 bead to $5 \mathrm{mg}$ of beads. However, on a large scale, this reaction resulted in the formation of an unidentified gummy residue that was released from the beads upon HF-mediated compound cleavage. Separating the product from this gummy residue by flash chromatography was problematic, resulting in a low isolated yield. TLC (hexanes:EtOAc 1:2) $\mathrm{R}_{\mathrm{f}}=0.24$; FTIR (film, $\mathrm{cm}^{-1}$ ) 3432, 2932, 2859, 1780, 1695, 1455, 1391, 1354, 1213, 1112, 1051; ${ }^{1} \mathrm{H}$ NMR (500 $\left.\mathrm{MHz}_{\mathrm{CDCl}}\right) \square 7.35-7.26(\mathrm{~m}, 3 \mathrm{H}, \mathrm{PhH}), 7.21(\operatorname{app~d}, J=7 \mathrm{~Hz}, 2 \mathrm{H}, \mathrm{PhH}), 6.93(\mathrm{~d}, J=10 \mathrm{~Hz}$, $\left.1 \mathrm{H}, \mathrm{C}_{5},-\mathbf{H}\right), 5.94\left(\mathrm{~d}, J=10.5 \mathrm{~Hz}, 1 \mathrm{H}, \mathrm{C}_{4},-\mathbf{H}\right), 5.22\left(\mathrm{t}, J=8 \mathrm{~Hz}, 1 \mathrm{H}, \mathrm{C}_{6}=\mathrm{CHCH}_{2}\right), 4.77-4.70(\mathrm{~m}$, $\left.1 \mathrm{H}, \mathrm{C}_{4}-\mathbf{H}\right), 4.73\left(\mathrm{~d}, J=8.5 \mathrm{~Hz}, 1 \mathrm{H}, \mathrm{C}_{2},-\mathbf{H}\right), 4.32-4.26\left(\mathrm{~m}, 2 \mathrm{H}, \mathrm{C}_{5}-\mathbf{H H}\right.$ and $\left.\mathrm{NCOCHCH}_{3}\right), 4.18$ $\left(\mathrm{dd}, J=8.5,2.5 \mathrm{~Hz}, 1 \mathrm{H}, \mathrm{C}_{5}-\mathrm{HH}\right), 3.66\left(\mathrm{t}, J=6.5 \mathrm{~Hz}, 2 \mathrm{H}, \mathrm{CH}_{2} \mathrm{OH}\right), 3.28(\mathrm{dd}, J=13,3.5 \mathrm{~Hz}, 1 \mathrm{H}$, $\left.\mathrm{C}_{4}-\mathrm{CHHPh}\right), 2.81\left(\mathrm{dd}, J=13,10 \mathrm{~Hz}, 1 \mathrm{H}, \mathrm{C}_{4}-\mathrm{CHHPh}\right), 2.32-2.26\left(\mathrm{~m}, 2 \mathrm{H}, \mathrm{C}_{6}=\mathrm{CHCH}_{2}\right), 1.62-$ $1.40\left(\mathrm{~m}, 6 \mathrm{H}, \mathrm{CH}_{2} \mathrm{CH}_{2} \mathrm{CH}_{2} \mathrm{CH}_{2} \mathrm{OH}\right), 1.41\left(\mathrm{~d}, J=6.5 \mathrm{~Hz}, 3 \mathrm{H}, \mathrm{CH}_{3}\right){ }^{13} \mathrm{C} \mathrm{NMR}\left(100 \mathrm{MHz}, \mathrm{CDCl}_{3}\right) \square$ 193.4, 173.9, 153.3, 146.9, 141.9, 135.2, 129.4, 128.9, 127.3, 122.1, 121.6, 81.0, 66.3, 62.8, 55.5, $39.8,38.0,32.5,28.7,27.5,25.5,13.7$; HRMS $\left(\mathrm{ES}^{+}\right)$calculated for $\mathrm{C}_{24} \mathrm{H}_{29} \mathrm{NO}_{6}(\mathrm{M}+\mathrm{H})^{+}$: 428.2073, found: 428.2061 .

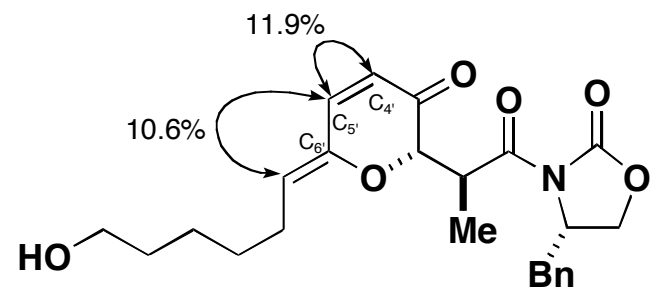

Selected nuclear Overhauser enhancements observed by one-dimensional nOe experiment (irradiation of $\mathrm{C}_{5}, \mathbf{H}$ ) 


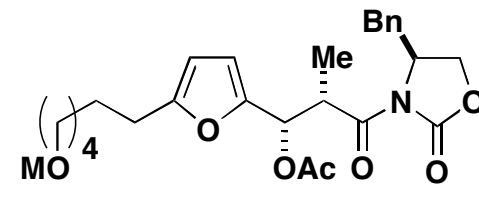

34

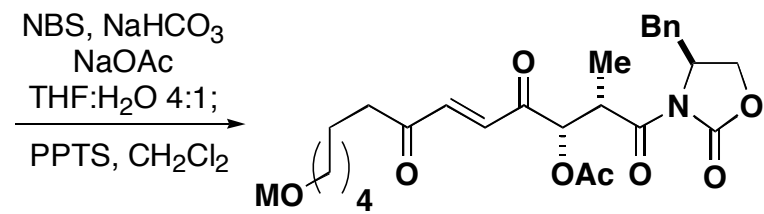

37

Macrobead-bound-1-((4S)-4-Benzyl-2-oxo-oxazolidin-3-yl)-(2S,3S)-3-acetoxy-13-hydroxy2-methyl-tridec-5-ene-1,4,7-trione (37)

Light yellow beads $34(0.090 \mathrm{~g}, 0.482 \mathrm{meq} . / \mathrm{g}, 0.043 \mathrm{mmol})$ were treated with the same reaction conditions and washing protocols described above for the transformation of $\mathbf{3 2} \rightarrow \mathbf{3 5}$. Solvent was then removed in vacuo to yield $\mathbf{3 7}$ as light yellow beads. $5.2 \mathrm{mg}$ of this resin was then treated with HF/Pyridine cleavage conditions (see General Methods) to yield crude alcohol 37 $_{\mathrm{M}=\mathbf{H}}$ with LCMS purity $>90 \%\left(\square_{214}, \mathrm{t}_{\mathrm{R}} 8.12 \mathrm{~min}\right) .83 .8 \mathrm{mg}$ of this resin was then treated with $\mathrm{HF} /$ Pyridine cleavage conditions and the crude product was purified by flash chromatography $\left(\mathrm{SiO}_{2}\right.$, hexanes:EtOAc 1:1 $\rightarrow$ 1:2) to afford alcohol $\mathbf{3 7}_{\mathbf{M = H}}$ as a yellow oil [15.8 $\mathrm{mg}, 0.0324$ mmol, 0.387 meq./g, Theoretical yield 0.478 meq./g, $81 \%$ from 34). TLC (hexanes:EtOAc 1:2) $\mathrm{R}_{\mathrm{f}}=0.23$; FTIR (film, $\mathrm{cm}^{-1}$ ) 3539, 2934, 2860, 1779, 1746, 1691, 1454, 1390, 1220, 1108, 1047; ${ }^{1} \mathrm{H}$ NMR $\left(500 \mathrm{MHz}, \mathrm{CDCl}_{3}\right) \square 7.35-7.27(\mathrm{~m}, 3 \mathrm{H}, \mathrm{PhH}), 7.19$ (app d, $\left.J=6 \mathrm{~Hz}, 2 \mathrm{H}, \mathrm{PhH}\right), 7.16(\mathrm{~d}$, $J=15.5 \mathrm{~Hz}, 1 \mathrm{H}$, vinylC-H), $7.03(\mathrm{~d}, J=15.5 \mathrm{~Hz}, 1 \mathrm{H}$, vinylC-H $), 5.73(\mathrm{~d}, J=5 \mathrm{~Hz}, 1 \mathrm{H}$, CHOAc), 4.67-4.61 (m, 1H, C $4-\mathbf{H}), 4.33-4.27\left(\mathrm{~m}, 2 \mathrm{H}, \mathrm{C}_{5}-\mathbf{H H}\right.$ and $\left.\mathrm{NCOCHCH}_{3}\right), 4.21(\mathrm{dd}, J=9$, $\left.2.5 \mathrm{~Hz}, 1 \mathrm{H}, \mathrm{C}_{5}-\mathrm{HH}\right), 3.64$ (t, $\left.J=6.5 \mathrm{~Hz}, 2 \mathrm{H}, \mathrm{CH}_{2} \mathrm{OH}\right), 3.24$ (dd, $J=13,3 \mathrm{~Hz}, 1 \mathrm{H}, \mathrm{C}_{4}-\mathrm{CHHPh}$ ), $2.79\left(\mathrm{dd}, J=13,10 \mathrm{~Hz}, 1 \mathrm{H}, \mathrm{C}_{4}-\mathrm{CHHPh}\right), 2.66\left(\mathrm{t}, J=7 \mathrm{~Hz}, 2 \mathrm{H}, \mathrm{COCH}_{2}\right), 2.18\left(\mathrm{~s}, 3 \mathrm{H}, \mathrm{COCH}_{3}\right)$, $1.66\left(\mathrm{~m}, 2 \mathrm{H}, \mathrm{CH}_{2} \mathrm{CH}_{2} \mathrm{OH}\right), 1.57\left(\mathrm{~m}, 2 \mathrm{H}, \mathrm{COCH}_{2} \mathrm{CH}_{2}\right), 1.42-1.32\left(\mathrm{~m}, 4 \mathrm{H}, \mathrm{CH}_{2} \mathrm{CH}_{2} \mathrm{CH}_{2} \mathrm{CH}_{2} \mathrm{OH}\right)$, $1.25\left(\mathrm{~d}, J=7.5 \mathrm{~Hz}, 3 \mathrm{H}, \mathrm{COCHCH}_{3}\right) ;{ }^{13} \mathrm{C} \mathrm{NMR}\left(100 \mathrm{MHz} \mathrm{CDCl}_{3}\right) \square 200.0,194.7,172.7,170.2$, 153.3, 137.8, 134.9, 132.1, 129.4, 129.0, 127.4, 76.9, 66.5, 62.8, 55.4, 41.8, 39.5, 37.8, 32.5, 28.8, 25.4, 23.5, 20.6, 12.0; HRMS $\left(\mathrm{ES}^{+}\right)$calculated for $\mathrm{C}_{26} \mathrm{H}_{33} \mathrm{NO}_{8}(\mathrm{M}+\mathrm{H})^{+}:$488.2284, found: 488.2275 .

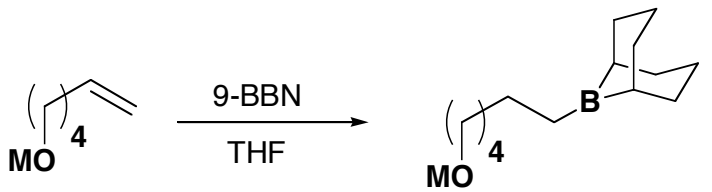

28

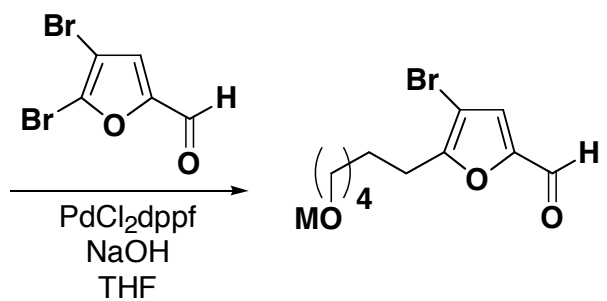

39

Macrobead-bound-4-Bromo-5-(6-hydroxy-hexyl)-furan-2-carbaldehyde (39)

Colorless beads 28 (500 mg) were washed with THF (2 x $10 \mathrm{~mL}$ x $10 \mathrm{~min}$ each) at rt and then resuspended in $15 \mathrm{~mL}$ THF. A $0.5 \mathrm{M}$ solution of $9-\mathrm{BBN}$ in THF $(10 \mathrm{~mL}, 5.0 \mathrm{mmol})$ was then added and the resulting colorless mixture was manually agitated and let stand at $\mathrm{rt}$ for $5 \mathrm{~h}$. The reaction solution was then removed via cannula and the colorless resin was washed thoroughly with THF ( 5 x $15 \mathrm{~mL}$ x $10 \mathrm{~min}$ each). To the resin was then added solid $\mathrm{PdCl}_{2} \mathrm{dppf}$ (10.2 mg, $0.0125 \mathrm{mmol}$ ), 4,5-dibromo-2-furaldehyde (635 $\mathrm{mg}, 2.5 \mathrm{mmol})$ via cannula as a solution in THF (6.25 mL), and a $1 \mathrm{M}$ aq. solution of $\mathrm{NaOH}(1.25 \mathrm{~mL}, 1.25 \mathrm{mmol})$. The resulting orange reaction mixture was sealed under a cloud of argon and heated at $65{ }^{\circ} \mathrm{C}$ with periodic manual agitation for $18 \mathrm{~h}$ (reaction mixture turned dark brown). The dark orange resin 
was then isolated by filtration and washed as follows, $4 \times\left(5 \times\right.$ THF, $5 \mathrm{x} \mathrm{H}_{2} \mathrm{O}, 5 \mathrm{x}$ THF, THF: $\mathrm{H}_{2} \mathrm{O}$ 3:1 x $30 \mathrm{~min}$ ), $5 \times \mathrm{THF}$, THF x $30 \mathrm{~min}, 5 \times \mathrm{CH}_{2} \mathrm{Cl}_{2}, \mathrm{CH}_{2} \mathrm{Cl}_{2} \times 30 \mathrm{~min}, 5 \times$ anh. $\mathrm{CH}_{2} \mathrm{Cl}_{2}$, anh. $\mathrm{CH}_{2} \mathrm{Cl}_{2} \times 30 \mathrm{~min}$, and then the solvent was removed in vacuo to yield $525 \mathrm{mg}$ of dark orange product resin 39. $5 \mathrm{mg}$ of this resin was then treated with HF/Pyridine cleavage conditions (see General Methods) to yield crude alcohol 39 ${ }_{\mathrm{M}=\mathbf{H}}$ with LCMS purity $88 \%\left(\square_{214}, \mathrm{t}_{\mathrm{R}} 6.40 \mathrm{~min}\right) .75 .4$ mg of this resin was then treated with HF/Pyridine cleavage conditions and the crude product was purified by flash chromatography $\left(\mathrm{SiO}_{2}\right.$, hexanes:EtOAc 1:2) to afford alcohol $\mathbf{3 9}_{\mathrm{M}=\mathrm{H}}$ as a yellow oil (3.9 mg, 0.188 meq./g, 19\% over two steps based on estimated maximum meq. Si/g). TLC (hexanes:EtOAc 1:1) $\mathrm{R}_{\mathrm{f}}=0.26$; FTIR (film, $\mathrm{cm}^{-1}$ ) 3401, 2932, 2858, 1683, 1521, 1462, 1393, 1285, 1119; ${ }^{1} \mathrm{H}$ NMR (500 MHz, $\left.\mathrm{CDCl}_{3}\right) \square 9.51$ (s, 1H, CHO), 7.19 (s, 1H, C $\left.\mathrm{C}_{3}-\mathbf{H}\right), 3.64(\mathrm{t}$, $\left.J=7.0 \mathrm{~Hz}, 2 \mathrm{H}, \mathrm{CH}_{2} \mathrm{OH}\right), 2.76\left(\mathrm{t}, J=7.5 \mathrm{~Hz}, 2 \mathrm{H}, \operatorname{FurCH}_{2}\right), 1.73\left(\mathrm{~m}, 2 \mathrm{H}\right.$, furCH$\left._{2} \mathrm{CH}_{2}\right), 1.57(\mathrm{~m}$, $\left.2 \mathrm{H}, \mathrm{CH}_{2} \mathrm{CH}_{2} \mathrm{OH}\right), 1.39$ (m, 4H, $\left.\mathrm{CH}_{2} \mathrm{CH}_{2} \mathrm{CH}_{2} \mathrm{CH}_{2} \mathrm{OH}\right) ;{ }^{13} \mathrm{C}$ NMR $\left(100 \mathrm{MHz}, \mathrm{CDCl}_{3}\right) \square$ 176.6, 160.3, 150.9, 124.3, 99.3, 62.8, 32.5, 28.8, 27.2, 26.5, 25.3; HRMS $\left(\mathrm{ES}^{+}\right)$calculated for $\mathrm{C}_{11} \mathrm{H}_{15} \mathrm{BrO}_{3}(\mathrm{M}+\mathrm{H})^{+}:$275.0283, found: 275.0282 .

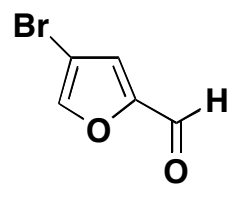

51

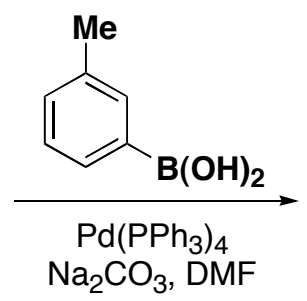

$$
\mathrm{Na}_{2} \mathrm{CO}_{3}, \mathrm{DMF}
$$

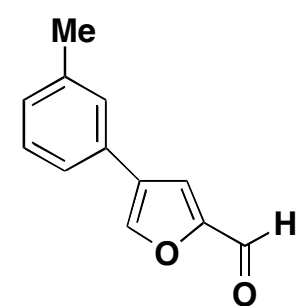

52

\section{4-m-Tolyl-furan-2-carbaldehyde (52)}

To a stirred mixture of 4-bromo-2-furaldehyde (ABCR, $5.070 \mathrm{~g}, 29.0 \mathrm{mmol}$ ) and $\mathrm{Pd}\left(\mathrm{PPh}_{3}\right)_{4}(0.869 \mathrm{mmol}, 1.004 \mathrm{~g})$ in DMF $(132 \mathrm{~mL})$ at $\mathrm{rt}$ under argon was added sodium carbonate $(72.4 \mathrm{mmol}, 7.68 \mathrm{~g})$ as a solution in a minimum amount of water $(20 \mathrm{~mL})$, followed by 3 -methylbenzeneboronic acid $(30.4 \mathrm{mmol}, 4.14 \mathrm{~g})$. The resulting light yellow reaction mixture was fitted with a reflux condensor and heated to $105-110{ }^{\circ} \mathrm{C}$ with vigorous stirring for $22.5 \mathrm{~h}$ (reaction mixture became very dark as reaction progressed). The dark brown reaction mixture was then cooled to $\mathrm{rt}$, filtered over a glass frit, diluted with water $(100 \mathrm{~mL})$ and $\mathrm{Et}_{2} \mathrm{O}(150 \mathrm{~mL})$ and transferred to a separatory funnel. The layers were then separated and the aqueous/DMF layer was extracted with $\mathrm{Et}_{2} \mathrm{O}(3 \times 100 \mathrm{~mL})$. The combined organic fractions were washed with water $(60 \mathrm{~mL})$, brine:water 1:1 $(60 \mathrm{~mL})$, and brine $(60 \mathrm{~mL})$, dried over magnesium sulfate, and concentrated in vacuo. Purification by flash chromatography $\left(\mathrm{SiO}_{2}\right.$; hexanes:EtOAc 50:1 $\rightarrow$ 30:1, column was repeated on fractions containing Pd-discoloration) afforded the desired biaryl product 52 as a yellow/orange oil (4.5 g, $24.2 \mathrm{mmol}, 83 \%$ ). TLC (hexanes:EtOAc 20:1 x 3 cycles) $\mathrm{R}_{\mathrm{f}}=0.27$; FTIR (film, $\mathrm{cm}^{-1}$ ) 3131, 3027, 2920, 2827, 1681, 1613, 1518, 1478, 1349, 1148; ${ }^{1} \mathrm{H}$ NMR (500 MHz, $\left.\mathrm{CDCl}_{3}\right) \square 9.70$ (s, 1H, CHO), 7.94 (s, 1H, C $\left.-\mathbf{H}\right), 7.51$ (s, 1H, C 3 -H), 7.31 (m, 3H, ArH), $7.16(\mathrm{~m}, 1 \mathrm{H}, \mathrm{ArH}), 2.40$ (s, 3H, $\left.\mathrm{CH}_{3}\right) ;{ }^{13} \mathrm{C} \mathrm{NMR}\left(100 \mathrm{MHz}, \mathrm{CDCl}_{3}\right) \square 178.0$, 153.5, 143.7, 138.8, 130.3, $129.4,129.0,128.9,126.7,123.0,119.0,21.4$; $\mathrm{HRMS}_{\left(\mathrm{ES}^{+}\right)}$ calculated for $\mathrm{C}_{12} \mathrm{H}_{10} \mathrm{O}_{2}(\mathrm{M}+\mathrm{H})^{+}: 187.0759$, found: 187.0753 . 


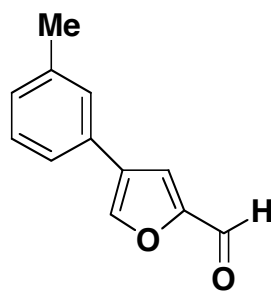

52

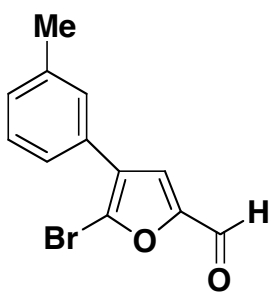

53

\section{5-Bromo-4-m-tolyl-furan-2-carbaldehyde (53)}

To DMF (19.3 $\mathrm{mL})$ stirred at -60 to $-55^{\circ} \mathrm{C}$ under argon was added bromine $(48.3 \mathrm{mmol}$, $2.48 \mathrm{ml}$ ) dropwise over $15 \mathrm{~min}$. The resulting red/orange slurry (solidification occurred upon bromine addition) was warmed to $-25{ }^{\circ} \mathrm{C}$ over 30 min to yield a bright orange solution (maintained at $-25^{\circ} \mathrm{C}$ ). In a separate flask, 4- $m$-tolyl-furan-2-carbaldehyde (52) was dissolved in DMF (19.3 mL) and stirred at $\mathrm{rt}$ under argon. To this solution was added the $\mathrm{Br}_{2} / \mathrm{DMF}$ solution dropwise via cannula over $45 \mathrm{~min}$. The resulting dark orange/brown solution was stirred for an additional $15 \mathrm{~min}$ and then transferred to a separatory funnel and extracted with $8.5 \%$ ethyl acetate/hexanes $(5 \times 100 \mathrm{~mL}, 2 \times 50 \mathrm{~mL})$. The combined extracts were then concentrated in vacuo and the resulting orange $\mathrm{DMF}$ solution was dissolved in $\mathrm{Et}_{2} \mathrm{O}(200 \mathrm{~mL})$ and washed with water (1 x $40 \mathrm{~mL}, 1 \times 20 \mathrm{~mL})$ (ethereal layer turned light yellow) and brine (1 x $20 \mathrm{~mL})$, dried over sodium sulfate, and concentrated in vасио. The crude product was azeotropically dried (benzene $30 \mathrm{~mL}$, rotary evaporation) to yield $4.3 \mathrm{~g}$ of an orange oil, which was purified by flash chromatography $\left(\mathrm{SiO}_{2}\right.$, hexanes:EtOAc 100:1 $\rightarrow$ 50:1) to yield $\mathbf{5 3}$ as a light brown solid (3.9 g, 14.7 mmol, 76\%). TLC (hexanes:EtOAc 20:1 x 3 cycles) $\mathrm{R}_{\mathrm{f}}=0.30$; FTIR (film, $\mathrm{cm}^{-1}$ ) 3106, 2921, 2824, 1684, 1611, 1578, 1512, 1473, 1370, 1342, 1302, 1167; ${ }^{1} \mathrm{H} \mathrm{NMR}\left(500 \mathrm{MHz}, \mathrm{CDCl}_{3}\right)$ $\square 9.59$ (s, 1H, CHO), 7.42-7.33 (m, 3H, ArH), 7.39 (s, 1H, $\left.\mathrm{C}_{3}-\mathbf{H}\right) 7.21$ (app d, J = 7.5 Hz, 1H, $\mathrm{ArH}), 2.42$ (s, 3H, $\left.\mathrm{CH}_{3}\right) ;{ }^{13} \mathrm{C}$ NMR (100 MHz, $\left.\mathrm{CDCl}_{3}\right) \square$ 176.6, 153.6, 138.6, 129.7, 129.2, 128.7, 128.1, 127.9, 127.8, 124.5, 121.8, 21.4; HRMS $\left(\mathrm{ES}^{+}\right)$calculated for $\mathrm{C}_{12} \mathrm{H}_{9} \mathrm{BrO}_{2}(\mathrm{M}+\mathrm{H})^{+}$: 264.9864, found: 264.9871 .

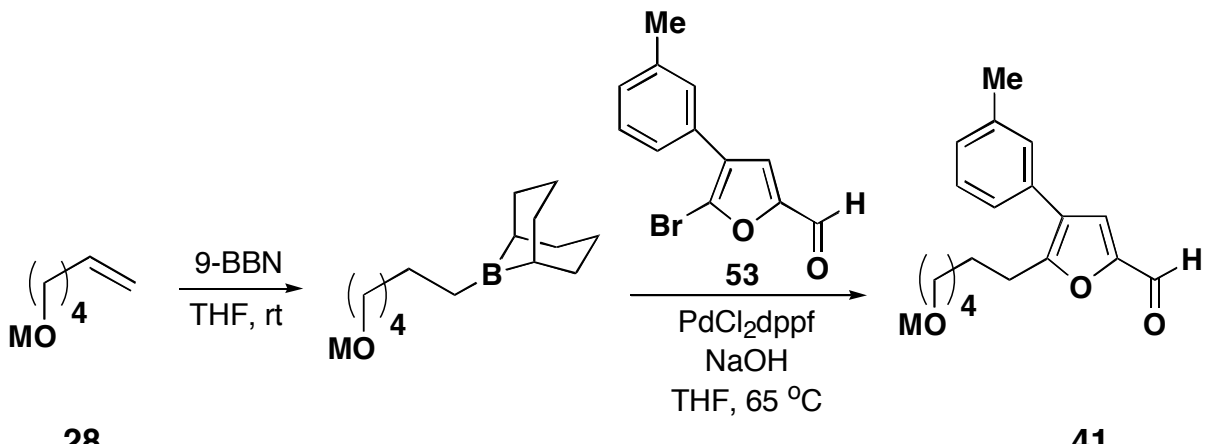

Macrobead-bound-5-(6-Hydroxy-hexyl)-4-m-tolyl-furan-2-carbaldehyde (41)

Colorless beads 28 (667 mg, max theoretical loading $1.3 \mathrm{meq} / \mathrm{g}, 0.867 \mathrm{mmol}$ ) were washed with THF (1 x $30 \mathrm{~mL}$ x $10 \mathrm{~min}, 1 \times 20 \mathrm{~mL}$ x $10 \mathrm{~min})$ at $\mathrm{rt}$ and then resuspended in 20.1 $\mathrm{mL}$ THF. A $0.5 \mathrm{M}$ solution of $9-\mathrm{BBN}$ in THF $(13.3 \mathrm{~mL}, 6.67 \mathrm{mmol})$ was then added and the resulting colorless mixture was manually agitated and let stand at $\mathrm{rt}$ for $5 \mathrm{~h}$. The reaction solution was then removed via cannula and the colorless resin was washed thoroughly with THF (5 x $15 \mathrm{~mL}$ x 5-10 min each). To the resin was then added solid $\mathrm{PdCl}_{2} \mathrm{dppf}$ (8.2 $\mathrm{mg}, 0.0075$ 
mmol), 4- $m$-MePh-5-bromofuraldehyde 53 (884 mg, $3.34 \mathrm{mmol}$ ) via cannula as a solution in THF $(8.3 \mathrm{~mL})$, and a $1 \mathrm{M}$ aq. solution of $\mathrm{NaOH}(1.67 \mathrm{~mL}, 1.67 \mathrm{mmol})$. The resulting orange reaction mixture was sealed under a cloud of argon and heated at $65{ }^{\circ} \mathrm{C}$ with periodic manual agitation for $22 \mathrm{~h}$ (reaction mixture turned dark brown). The yellow/orange resin was then isolated by filtration and washed as follows, $5 \mathrm{x}$ THF, $5 \mathrm{x} \mathrm{H}_{2} \mathrm{O}, 5 \mathrm{x}$ THF, THF: $\mathrm{H}_{2} \mathrm{O}$ 3:1 $\mathrm{x} 1 \mathrm{~h}, 2 \mathrm{x}$ (5 x THF, THF: $\mathrm{H}_{2} \mathrm{O} 3: 1 \times 1 \mathrm{~h}$ ), 5 x THF, THF x $20 \mathrm{~min}, 5 \times \mathrm{CH}_{2} \mathrm{Cl}_{2}, \mathrm{CH}_{2} \mathrm{Cl}_{2}$ x $20 \mathrm{~min}, 5$ x anh. $\mathrm{CH}_{2} \mathrm{Cl}_{2}$, anh. $\mathrm{CH}_{2} \mathrm{Cl}_{2} \times 20 \mathrm{~min}$, and then the solvent was removed in vacuo to yield $761.2 \mathrm{mg}$ of yellow/orange product resin 41. $5.2 \mathrm{mg}$ of this resin was then treated with HF/Pyridine cleavage conditions (see General Methods) to yield crude alcohol $\mathbf{4 1}_{\mathbf{M = H}}$ with LCMS purity $>90 \%\left(\square_{214}, \mathrm{t}_{\mathrm{R}}\right.$ $8.07 \mathrm{~min}$ ). $75 \mathrm{mg}$ of this resin was then treated with HF/Pyridine cleavage conditions and crude product was purified by flash chromatography $\left(\mathrm{SiO}_{2}\right.$, hexanes:EtOAc 1:1) to afford alcohol $\mathbf{4 1}_{\mathrm{M}=\mathrm{H}}$ as a yellow oil (11.7 mg, $0.545 \mathrm{meq} . / \mathrm{g}$ loading level). TLC (hexanes:EtOAc 1:1) $\mathrm{R}_{\mathrm{f}}=$ 0.29; FTIR (film, $\mathrm{cm}^{-1}$ ) 3433, 2931, 2858, 1678, 1611, 1526, 1483, 1333, 1122; ${ }^{1} \mathrm{H}$ NMR (500 $\left.\mathrm{MHz}, \mathrm{CDCl}_{3}\right) \square 9.57$ (s, 1H, CHO), 7.34-7.30 (m, 2H, ArH and $\left.\mathrm{C}_{3}-\mathbf{H}\right)$, 7.19-7.14 (m, 3H, ArH), $3.61\left(\mathrm{t}, J=6.5 \mathrm{~Hz}, 2 \mathrm{H}, \mathrm{CH}_{2} \mathrm{OH}\right), 2.86\left(\mathrm{t}, J=7.5 \mathrm{~Hz}, 2 \mathrm{H}, \mathrm{FurCH}_{2}\right), 2.40\left(\mathrm{~s}, 3 \mathrm{H}, \mathrm{CH}_{3}\right), 1.77(\mathrm{~m}$, $2 \mathrm{H}$, FurCH $\left.\mathrm{CH}_{2}\right), 1.54\left(\mathrm{~m}, 2 \mathrm{H}, \mathrm{CH}_{2} \mathrm{CH}_{2} \mathrm{OH}\right), 1.37$ (m, 4H, $\left.\mathrm{CH}_{2} \mathrm{CH}_{2} \mathrm{CH}_{2} \mathrm{CH}_{2} \mathrm{OH}\right) ;{ }^{13} \mathrm{C}$ NMR (100 $\left.\mathrm{MHz}, \mathrm{CDCl}_{3}\right) \square 177.1,159.2,150.9,138.6,132.1,128.7,128.6,128.3,124.9,124.8,123.5,62.8$, 32.5, 29.0, 27.9, 27.1, 25.3, 21.5; HRMS $\left(\mathrm{ES}^{+}\right)$calculated for $\mathrm{C}_{18} \mathrm{H}_{22} \mathrm{O}_{3}(\mathrm{M}+\mathrm{H})^{+}$: 287.1647 , found: 287.1647.

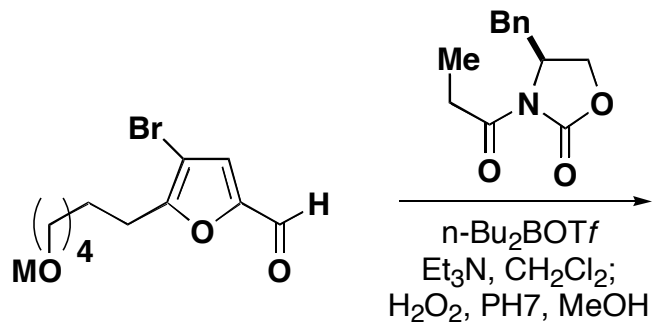

39

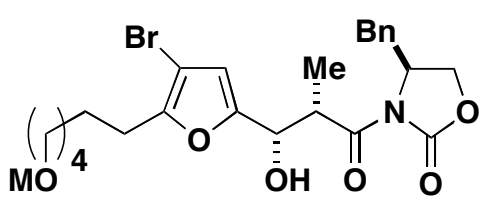

42

Macrobead-bound-(4S)-4-Benzyl-3-\{(3S,2S)-3-[4-bromo-5-(6-hydroxy-hexyl)-furan-2-yl]-3hydroxy-2-methyl-propionyl $\}$-oxazolidin-2-one (42)

Light yellow beads 39 (358 mg, 0.188 meq. $/ \mathrm{g}, 0.0673$ meq.) were treated with the same reaction conditions used for the transformation of $\mathbf{3 0} \rightarrow \mathbf{3 3}$. After washing, solvent was removed in vacuo to yield $381 \mathrm{mg}$ of light yellow product resin 42. $5.2 \mathrm{mg}$ of this resin was then treated with HF/Pyridine cleavage conditions (see General Methods) to yield crude alcohol $\mathbf{4 2}_{\mathrm{M}=\mathbf{H}}$ with LCMS purity $>90 \%\left(\square_{214}, \mathrm{t}_{\mathrm{R}} 8.56 \mathrm{~min}\right)$. $75.2 \mathrm{mg}$ of this resin was then treated with HF/Pyridine cleavage conditions and the crude product was purified by flash chromatography $\left(\mathrm{SiO}_{2}\right.$, hexanes:EtOAc 1:1 $\rightarrow$ 1:2) to afford alcohol $\mathbf{4 2}_{\mathrm{M=H}}$ as a light yellow oil (8.8 $\mathrm{mg}, 0.0173 \mathrm{mmol}$, 0.230 meq./g, Theoretical yield 0.180 meq./g, $>95 \%$ from 39). TLC (hexanes:EtOAc 1:2) $R_{\mathrm{f}}=$ 0.46; FTIR (film, cm ${ }^{-1}$ ) 3446, 2932, 2858, 1781, 1696, 1454, 1386, 1210, 1109, 1014; ${ }^{1} \mathrm{H}$ NMR $\left(500 \mathrm{MHz}, \mathrm{CDCl}_{3}\right) \square 7.36-7.28(\mathrm{~m}, 3 \mathrm{H}, \mathrm{PhH}), 7.20(\operatorname{app~d}, J=7 \mathrm{~Hz}, 2 \mathrm{H}, \mathrm{PhH}), 6.28(\mathrm{~s}, 1 \mathrm{H}$, FurH), $5.0(\mathrm{~m}, 1 \mathrm{H}$, FurCHOH$), 4.67\left(\mathrm{~m}, 1 \mathrm{H}, \mathrm{C}_{4}-\mathbf{H}\right), 4.20\left(\operatorname{app~d}, J=5.5 \mathrm{~Hz}, 2 \mathrm{H}, \mathrm{C}_{5}-\mathbf{H H}\right), 4.13$ $\left(\mathrm{dq}, J=7,5 \mathrm{~Hz}, 1 \mathrm{H}, \mathrm{COCHCH}_{3}\right), 3.62\left(\mathrm{t}, J=6 \mathrm{~Hz}, 2 \mathrm{H}, \mathrm{CH}_{2} \mathrm{OH}\right), 3.24(\mathrm{dd}, J=13.5,3 \mathrm{~Hz}, 1 \mathrm{H}$, $\mathrm{C}_{4}-\mathrm{CHHPh}$ ), 3.12 (br d, $J=3.5 \mathrm{~Hz}, 1 \mathrm{H}$, FurCHOH), 2.79 (dd, $J=13,9 \mathrm{~Hz}, 1 \mathrm{H}, \mathrm{C}_{4}-\mathrm{CHHPh}$ ), $2.61\left(\mathrm{t}, J=7.5 \mathrm{~Hz}, 2 \mathrm{H}, \mathrm{FurCH}_{2}\right), 1.62\left(\mathrm{~m}, 2 \mathrm{H}, \mathrm{FurCH}_{2} \mathrm{CH}_{2}\right), 1.56\left(\mathrm{~m}, 2 \mathrm{H}, \mathrm{CH}_{2} \mathrm{CH}_{2} \mathrm{OH}\right), 1.4-1.32$ $\left(\mathrm{m}, 4 \mathrm{H}, \mathrm{CH}_{2} \mathrm{CH}_{2} \mathrm{CH}_{2} \mathrm{CH}_{2} \mathrm{OH}\right), 1.32\left(\mathrm{~d}, J=7 \mathrm{~Hz}, 3 \mathrm{H}, \mathrm{CH}_{3}\right) ;{ }^{13} \mathrm{C}$ NMR $\left(100 \mathrm{MHz}, \mathrm{CDCl}_{3}\right) \square$ 
176.2, 152.8, 152.5, 152.3, 134.8, 129.4, 129.0, 127.5, 110.6, 96.4, 68.4, 66.3, 62.8, 55.1, 42.2, $37.8,32.5,28.5,27.4,25.8,25.2,11.9$; HRMS $\left(\mathrm{ES}^{+}\right)$calculated for $\mathrm{C}_{24} \mathrm{H}_{30} \mathrm{BrNO}_{6}(\mathrm{M}+\mathrm{Na})^{+}$: 530.1154, found: 530.1169 .

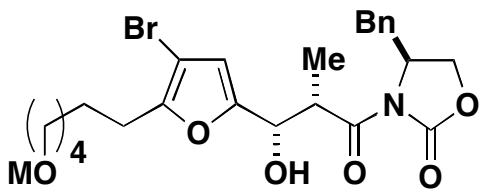

42

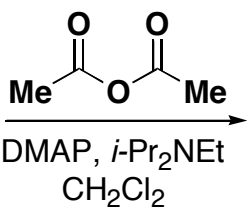

$\mathrm{CH}_{2} \mathrm{Cl}_{2}$

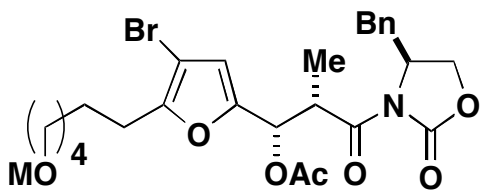

43

Macrobead-bound-(4S)-4-Benzyl-3-\{(3S,2S)-3-[4-bromo-5-(6-hydroxy-hexyl)-furan-2-yl]-3acetoxy-2-methyl-propionyl\}-oxazolidin-2-one (43)

Light yellow beads $42(180 \mathrm{mg}, 0.0414$ meq.) were treated with the same reaction conditions used for the transformation of $\mathbf{3 3} \rightarrow \mathbf{3 4}$. Solvent was removed in vacuo to yield 183 $\mathrm{mg}$ of light yellow product resin $43.5 .0 \mathrm{mg}$ of this resin was then treated with HF/Pyridine cleavage conditions (see General Methods) to yield crude alcohol $\mathbf{4 3}_{\mathrm{M}=\mathrm{H}}$ with LCMS purity $>90 \%\left(\square_{214}, \mathrm{t}_{\mathrm{R}} 8.94 \mathrm{~min}\right) .75 .3 \mathrm{mg}$ of this resin was then treated with HF/Pyridine cleavage conditions and the crude product was purified by flash chromatography $\left(\mathrm{SiO}_{2}\right.$, hexanes:EtOAc $1: 1 \rightarrow 1: 2)$ to afford alcohol $\mathbf{4 3}_{\mathrm{M}=\mathrm{H}}$ as a yellow oil $(8.5 \mathrm{mg}, 0.0154 \mathrm{mmol}, 0.205 \mathrm{meq} . \mathrm{g}$, Theoretical yield 0.228 meq./g, 90\% from 42). TLC (hexanes:EtOAc 1:1) $\mathrm{R}_{\mathrm{f}}=0.21$; FTIR (film, $\left.\mathrm{cm}^{-1}\right) 3535,2933,2859,1782,1745,1698,1454,1387,1223,1108,1018 ;{ }^{1} \mathrm{H}$ NMR (500 MHz, $\mathrm{CDCl}_{3}$ ) $\square$ 7.34-7.26 (m, 3H, PhH), 7.19 (app d, $\left.J=7.5 \mathrm{~Hz}, 2 \mathrm{H}, \mathrm{PhH}\right), 6.31$ (s, 1H, FurH), 6.11 (d, $J=7.5 \mathrm{~Hz}, 1 \mathrm{H}$, FurCHOAc), $4.55\left(\mathrm{~m}, 1 \mathrm{H}, \mathrm{C}_{4}-\mathbf{H}\right), 4.47\left(\mathrm{dq}, J=7,7 \mathrm{~Hz}, 1 \mathrm{H}, \mathrm{COCHCH}_{3}\right), 4.16$ (app d, $\left.J=4.5 \mathrm{~Hz}, 2 \mathrm{H}, \mathrm{C}_{5}-\mathrm{HH}\right), 3.62\left(\mathrm{t}, J=6.5 \mathrm{~Hz}, 2 \mathrm{H}, \mathrm{CH}_{2} \mathrm{OH}\right), 3.23\left(\mathrm{dd}, J=13,3 \mathrm{~Hz}, 1 \mathrm{H}, \mathrm{C}_{4^{-}}\right.$ CHHPh), 2.76 (dd, $\left.J=15,9.5 \mathrm{~Hz}, 1 \mathrm{H}, \mathrm{C}_{4}-\mathrm{CHHPh}\right), 2.60\left(\mathrm{t}, J=7 \mathrm{~Hz}, 2 \mathrm{H}, \operatorname{FurCH}_{2}\right), 2.09(\mathrm{~s}, 3 \mathrm{H}$, $\mathrm{COCH}_{3}$ ), 1.64-1.53 (m, 4H, $\mathrm{CH}_{2} \mathrm{CH}_{2} \mathrm{CH}_{2} \mathrm{CH}_{2} \mathrm{CH}_{2} \mathrm{OH}$ ), 1.40-1.30 (m, 4H, $\mathrm{CH}_{2} \mathrm{CH}_{2} \mathrm{CH}_{2} \mathrm{CH}_{2} \mathrm{OH}$ ), $1.32\left(\mathrm{~d}, J=6.5 \mathrm{~Hz}, 3 \mathrm{H}, \mathrm{CHCH}_{3}\right) ;{ }^{13} \mathrm{C}$ NMR $\left(100 \mathrm{MHz}, \mathrm{CDCl}_{3}\right) \square 173.1,170.0,153.1,153.1$, 149.4, 135.0, 129.4, 128.9, 127.4, 112.6, 96.4, 68.7, 66.3, 62.8, 55.3, 40.7, 37.8, 32.5, 28.6, 27.4, 25.9, 25.2, 20.9, 13.2; HRMS (ES ${ }^{+}$) calculated for $\mathrm{C}_{26} \mathrm{H}_{32} \mathrm{BrNO}_{7}(\mathrm{M}+\mathrm{Na})^{+}: 572.1260$, found: 572.1277 .

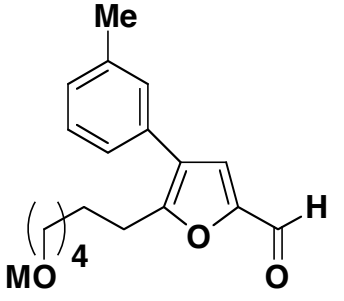

41

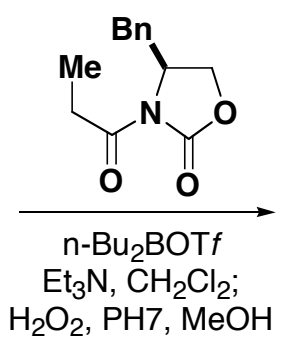

$\mathrm{H}_{2} \mathrm{O}_{2}, \mathrm{PH} 7, \mathrm{MeOH}$

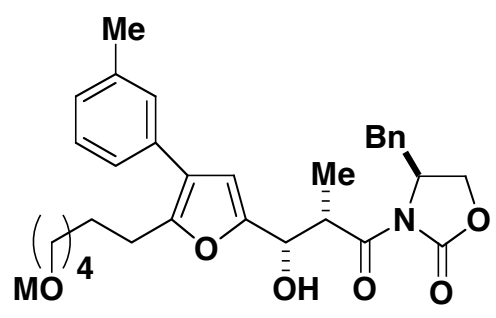

44

Macrobead-bound-(4S)-4-Benzyl-3-\{(3S,2S)-3-hydroxy-3-[5-(6-hydroxy-hexyl)-4-m-tolylfuran-2-yl]-2-methyl-propionyl\}-oxazolidin-2-one (44)

Light yellow beads 44 (400 $\mathrm{mg}, 0.218$ meq.) were treated with the same reaction conditions used for the transformation of $\mathbf{3 0} \rightarrow \mathbf{3 3}$. Solvent was removed in vacuo to yield 456 $\mathrm{mg}$ of light yellow product resin 44. $5.2 \mathrm{mg}$ of this resin was then treated with HF/Pyridine cleavage conditions (see General Methods) to yield crude diol $\mathbf{4 4}_{\mathrm{M}=\mathrm{H}}$ with LCMS purity $>90 \%$ 
$\left(\square_{214}, \mathrm{t}_{\mathrm{R}} 9.47 \mathrm{~min}\right) .75 .2 \mathrm{mg}$ of this resin was then treated with $\mathrm{HF} /$ Pyridine cleavage conditions and the crude product was purified by flash chromatography $\left(\mathrm{SiO}_{2}\right.$, hexanes:EtOAc 1:1 $\rightarrow$ 1:2) to afford diol $\mathbf{4 4}_{\mathrm{M=H}}$ as a yellow oil $(18.0 \mathrm{mg}, 0.0346 \mathrm{mmol}, 0.460 \mathrm{meq} . / \mathrm{g}$, Theoretical yield 0.484 meq./g, 95\% from 41). TLC (hexanes:EtOAc 1:1) $\mathrm{R}_{\mathrm{f}}=0.30$; FTIR (film, $\mathrm{cm}^{-1}$ ) 3446, 2932, 2858, 1782, 1696, 1605, 1455, 1386, 1210, 1109, 1051, 1015; $\left.{ }^{1} \mathrm{H} \mathrm{NMR} \mathrm{(500} \mathrm{MHz,} \mathrm{CDCl}_{3}\right)$ 77.36-7.06 (m, 9H, ArH and PhH), $6.41(\mathrm{~s}, 1 \mathrm{H}$, FurH), 5.07 (d, $J=4 \mathrm{~Hz}, 1 \mathrm{H}$, FurCHOH), 4.65 $\left(\mathrm{m}, 1 \mathrm{H}, \mathrm{C}_{4}-\mathbf{H}\right), 4.25-4.10\left(\mathrm{~m}, 3 \mathrm{H}, \mathrm{COCHCH}_{3}\right.$ and $\left.\mathrm{C}_{5}-\mathbf{H H}\right), 3.60\left(\mathrm{t}, J=7 \mathrm{~Hz}, 2 \mathrm{H}, \mathrm{CH}_{2} \mathrm{OH}\right), 3.25$ (dd, $\left.J=13.5,3 \mathrm{~Hz}, 1 \mathrm{H}, \mathrm{C}_{4}-\mathrm{CHHPh}\right), 3.10$ (br s, 1H, FurCHOH), 2.80 (dd, $J=13.5,9.5 \mathrm{~Hz}, 1 \mathrm{H}$, $\left.\mathrm{C}_{4}-\mathrm{CHHPh}\right), 2.75\left(\mathrm{t}, J=8 \mathrm{~Hz}, 2 \mathrm{H}, \mathrm{FurCH}_{2}\right), 2.37$ (s, 3H, $\left.\operatorname{ArCH}_{3}\right), 1.69\left(\mathrm{~m}, 2 \mathrm{H}, \mathrm{FurCH}_{2} \mathrm{CH}_{2}\right.$ ), $1.54\left(\mathrm{~m}, 2 \mathrm{H}, \mathrm{CH}_{2} \mathrm{CH}_{2} \mathrm{OH}\right), 1.39\left(\mathrm{~d}, J=7 \mathrm{~Hz}, 3 \mathrm{H}, \mathrm{COCHCH}_{3}\right) 1.35(\mathrm{~m}, 4 \mathrm{H}$, $\left.\mathrm{CH}_{2} \mathrm{CH}_{2} \mathrm{CH}_{2} \mathrm{CH}_{2} \mathrm{OH}\right) ;{ }^{13} \mathrm{C}$ NMR $\left(100 \mathrm{MHz}, \mathrm{CDCl}_{3}\right) \square 176.3,152.9,151.6,151.2,138.1,134.9$, 133.9, 129.4, 129.0, 128.4, 128.4, 127.5, 127.2, 124.7, 121.5, 108.5, 68.7, 66.2, 62.8, 55.2, 42.5, $37.8,32.5,28.8,28.1,26.7,25.2,21.5,12.1$; HRMS $\left(\mathrm{ES}^{+}\right)$calculated for $\mathrm{C}_{31} \mathrm{H}_{37} \mathrm{NO}_{6}\left(\mathrm{M}+\mathrm{NH}_{4}\right)^{+}$: 537.2965, Found: 537.2977.

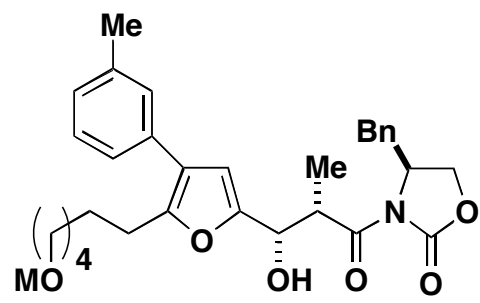

44

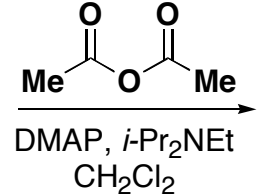

$\mathrm{CH}_{2} \mathrm{Cl}_{2}$

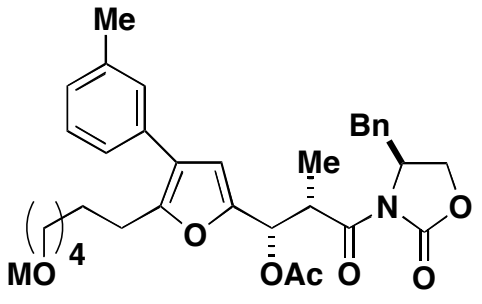

45

Macrobead-bound-(4S)-4-Benzyl-3-\{(3S,2S)-3-acetoxy-3-[5-(6-hydroxy-hexyl)-4-m-tolylfuran-2-yl]-2-methyl-propionyl $\}$-oxazolidin-2-one (45)

Light yellow beads 44 (180 mg, $0.460 \mathrm{meq} / \mathrm{g}, 0.083 \mathrm{meq}$.) were treated with the same reaction conditions used for the transformation of $\mathbf{3 3} \rightarrow \mathbf{3 4}$. Solvent was removed in vacuo to yield light yellow product resin 45. $5.2 \mathrm{mg}$ of this resin was then treated with HF/Pyridine cleavage conditions (see General Methods) to yield crude alcohol $\mathbf{4 5}_{\mathrm{M}=\mathrm{H}}$ with LCMS purity $>90 \%\left(\square_{214}, \mathrm{t}_{\mathrm{R}} 10.55 \mathrm{~min}\right) .75 .2 \mathrm{mg}$ of this resin was then treated with HF/Pyridine cleavage conditions and the crude product was purified by flash chromatography $\left(\mathrm{SiO}_{2}\right.$, hexanes:EtOAc $1: 1 \rightarrow 1: 2)$ to afford $\mathbf{4 5}_{\mathrm{M=H}}$ as a yellow oil $(16.0 \mathrm{mg}, 0.0285 \mathrm{mmol}, 0.379 \mathrm{meq} . / \mathrm{g}$, Theoretical yield 0.451 meq./g, 84\% from 44). TLC (hexanes:EtOAc 1:1) $\mathrm{R}_{\mathrm{f}}=0.26$; FTIR (film, $\mathrm{cm}^{-1}$ ) 3538, 3028, 2932, 2859, 1782, 1744, 1700, 1606, 1455, 1386, 1227, 1108, 1018; ${ }^{1} \mathrm{H}$ NMR (500 MHz, $\left.\mathrm{CDCl}_{3}\right) \square$ 7.34-7.06 (m, 9H, ArH and PhH), $6.44(\mathrm{~s}, 1 \mathrm{H}, \operatorname{FurH}), 6.19(\mathrm{~d}, J=8 \mathrm{~Hz}, 1 \mathrm{H}$, FurCHOAc), 4.59-4.50 (m, 2H, $\mathrm{C}_{4}-\mathbf{H}$ and $\left.\mathrm{COCHCH}_{3}\right), 4.16-4.10\left(\mathrm{~m}, 2 \mathrm{H}, \mathrm{C}_{5}-\mathbf{H H}\right), 3.60(\mathrm{t}, J=$ $\left.6.5 \mathrm{~Hz}, 2 \mathrm{H}, \mathrm{CH}_{2} \mathrm{OH}\right), 3.24\left(\mathrm{dd}, J=13,3 \mathrm{~Hz}, 1 \mathrm{H}, \mathrm{C}_{4}-\mathrm{CHHPh}\right), 2.77\left(\mathrm{dd}, J=13.5,10 \mathrm{~Hz}, 1 \mathrm{H}, \mathrm{C}_{4^{-}}\right.$ CHHPh), 2.74 (t, $\left.J=7.5 \mathrm{~Hz}, 2 \mathrm{H}, \mathrm{FurCH}_{2}\right), 2.36$ (s, 3H, $\left.\operatorname{ArCH}_{3}\right), 2.12$ (s, 3H, COCH $), 1.70-1.64$ (m, 2H, FurCH $\mathrm{CH}_{2}$ ), $1.54\left(\mathrm{~m}, 2 \mathrm{H}, \mathrm{CH}_{2} \mathrm{CH}_{2} \mathrm{OH}\right), 1.39-1.32\left(\mathrm{~m}, 4 \mathrm{H}, \mathrm{CH}_{2} \mathrm{CH}_{2} \mathrm{CH}_{2} \mathrm{CH}_{2} \mathrm{OH}\right), 1.36$ $(\mathrm{d}, J=6.5 \mathrm{~Hz}, 3 \mathrm{H}, \mathrm{COCHCH})_{3} ;{ }^{13} \mathrm{C} \mathrm{NMR}\left(100 \mathrm{MHz}, \mathrm{CDCl}_{3}\right) \square 173.4,170.1,153.1,151.7$, 148.7, 138.1, 135.1, 133.6, 129.4, 128.9, 128.4, 128.4, 127.4, 127.3, 124.6, 121.6, 110.5, 69.1, $\left.66.2,62.8,55.4,40.8,37.8,32.6,28.9,28.1,26.7,25.3,21.5,21.0,13.2 ; \mathrm{HRMS}_{(\mathrm{ES}}{ }^{+}\right)$calculated for $\mathrm{C}_{33} \mathrm{H}_{39} \mathrm{NO}_{7}(\mathrm{M}+\mathrm{Na})^{+}:$584.2624, found: 584.2609. 
<smiles>C[C@H](CCc1oc([C@@H](O)C(=O)N2C(=O)OCC2Cc2ccccc2)cc1Br)C(=O)O</smiles>

42

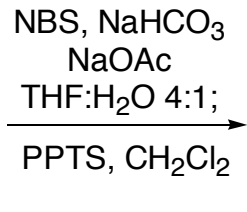<smiles>CC(C(=O)N1C(=O)OC[C@H]1Cc1ccccc1)[C@H]1OC(O)(CCC(C)(C)C)C(=O)C=C1Br</smiles>

46

Macrobead-bound-(4S)-4-Benzyl-3- $\{(2 S)-2-[(2 S, 6 R)-5$-bromo-6-hydroxy-6-(6-hydroxyhexyl)-3-oxo-3,6-dihydro-2H-pyran-2-yl]-propionyl\}-oxazolidin-2-one (46)

Light yellow beads 42 (0.090 g, 0.230 meq./g, $0.021 \mathrm{mmol})$ were treated with the same reaction conditions and washing protocol described above for the transformation of $\mathbf{3 2} \rightarrow \mathbf{3 5}$. Solvent was then removed in vacuo to yield $\mathbf{4 6}$ as light yellow beads. $5.2 \mathrm{mg}$ of this resin was then treated with HF/Pyridine cleavage conditions (see General Methods) to yield crude diol $\mathbf{4 6}_{\mathrm{M}=\mathbf{H}}$ with LCMS purity $90 \%\left(\square_{214}, \mathrm{t}_{\mathrm{R}} 8.14 \mathrm{~min}\right.$, epimeric ratio $\left.=9.4: 1\right) .87 .8 \mathrm{mg}$ of this resin was then treated with $\mathrm{HF} /$ Pyridine cleavage conditions and the crude product was purified by flash chromatography $\left(\mathrm{SiO}_{2}\right.$, hexanes:EtOAc $\left.1: 1 \rightarrow 1: 2\right)$ to afford diol $\mathbf{4 6}_{\mathrm{M}=\mathbf{H}}$ as a yellow oil (8.6 mg, 0.0164 mmol, 0.187 meq./g, Theoretical yield 0.229 meq./g, 82\% from 42). TLC (hexanes:EtOAc 1:2) $\mathrm{R}_{\mathrm{f}}=0.3$; FTIR (film, $\mathrm{cm}^{-1}$ ) 3452, 2933, 2860, 1781, 1695, 1605, 1455 , 1392, 1352, 1208, 1110, 1050; ${ }^{1} \mathrm{H}$ NMR (500 MHz, $\left.\mathrm{CDCl}_{3}\right) \square 7.35-7.25$ (m, 3H, PhH), 7.22-7.18 (m, 2H, PhH), $6.50\left(\mathrm{~s}, 1 \mathrm{H}, \mathrm{C}_{4}, \mathbf{H}\right), 4.92\left(\mathrm{~d}, J=8.5 \mathrm{~Hz}, 1 \mathrm{H}, \mathrm{C}_{2},-\mathbf{H}\right), 4.74\left(\mathrm{~m}, 1 \mathrm{H}, \mathrm{C}_{4}-\mathbf{H}\right), 4.30$ (app $\left.\mathrm{t}, J=8.5 \mathrm{~Hz}, 1 \mathrm{H}, \mathrm{C}_{5}-\mathrm{HH}\right), 4.19\left(\mathrm{dd}, J=9.5,2.5 \mathrm{~Hz}, 1 \mathrm{H}, \mathrm{C}_{5}-\mathrm{HH}\right), 4.12(\mathrm{dq}, J=8,7 \mathrm{~Hz}, 1 \mathrm{H}$, $\left.\mathrm{COCHCH}_{3}\right), 3.65\left(\mathrm{t}, J=7 \mathrm{~Hz}, 2 \mathrm{H}, \mathrm{CH}_{2} \mathrm{OH}\right), 3.25\left(\mathrm{dd}, J=13.5,3 \mathrm{~Hz}, 1 \mathrm{H}, \mathrm{C}_{4}-\mathrm{CHHPh}\right), 2.81$ (dd, $\left.J=13,10 \mathrm{~Hz}, 1 \mathrm{H}, \mathrm{C}_{4}-\mathrm{CHHPh}\right), 2.16\left(\mathrm{dt}, J=12.5,4 \mathrm{~Hz}, 1 \mathrm{H}, \mathrm{C}_{6}-\mathrm{CHH}\right), 1.93(\mathrm{dt}, J=10,4 \mathrm{~Hz}$ $\left.1 \mathrm{H}, \mathrm{C}_{6}, \mathrm{HH}\right), 1.58\left(\mathrm{~m}, 2 \mathrm{H}, \mathrm{CH}_{2} \mathrm{CH}_{2} \mathrm{OH}\right), 1.42-1.34\left(\mathrm{~m}, 6 \mathrm{H}, \mathrm{CH}_{2} \mathrm{CH}_{2} \mathrm{CH}_{2} \mathrm{CH}_{2} \mathrm{CH}_{2} \mathrm{OH}\right), 1.33$ (d, $J$ $\left.=7 \mathrm{~Hz}, 3 \mathrm{H}, \mathrm{CH}_{3}\right) ;{ }^{13} \mathrm{C} \mathrm{NMR}\left(100 \mathrm{MHz} \mathrm{CDCl}_{3}\right) \square$ 192.2, 174.3, 153.2, 148.3, 135.1, 131.0, 129.5, 129.0, 127.4, 98.1, 74.5, 66.3, 62.9, 55.3, 40.5, 38.3, 38.0, 32.5, 29.0, 25.4, 23.2, 13.6; HRMS (ES $)$ calculated for $\mathrm{C}_{24} \mathrm{H}_{30} \mathrm{BrNO}_{7}(\mathrm{M}+\mathrm{Na})^{+}:$546.1103, found: 546.1086.
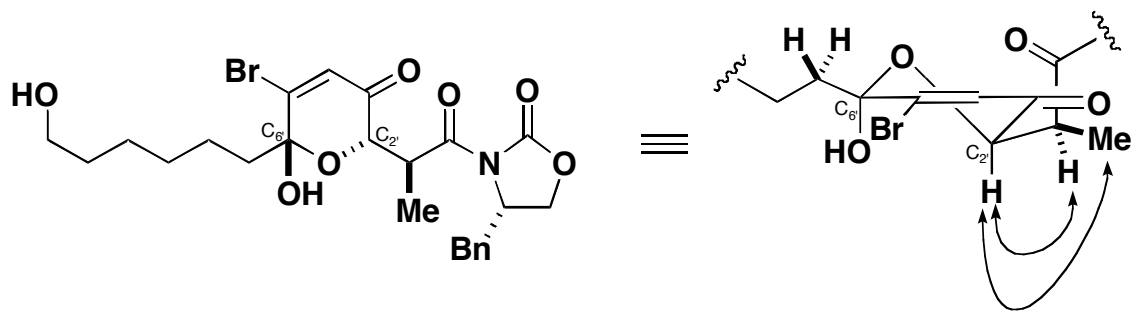

Selected nuclear Overhauser enhancements observed with a two-dimensional NOESY experiment. There was no nOe observed between $\mathrm{C}_{2},-\mathbf{H}$ and $\mathrm{C}_{6},-\mathrm{CHH}$ or $\mathrm{C}_{6},-\mathrm{CHH}$, consistent with the $R$ stereochemical assignment at $\mathrm{C}_{6}$. 
Burke, Berger, and Schreiber - Supporting Information Part A
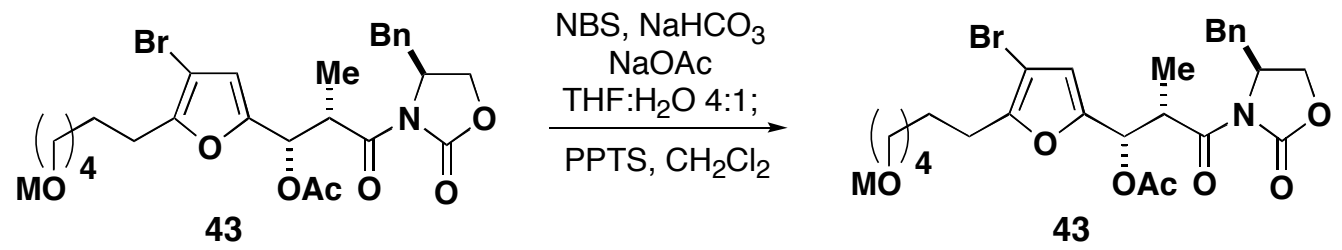

Macrobead-bound-(4S)-4-Benzyl-3-\{(3S,2S)-3-[4-bromo-5-(6-hydroxy-hexyl)-furan-2-yl]-3acetoxy-2-methyl-propionyl\}-oxazolidin-2-one (43)

Light yellow beads 43 ( $0.090 \mathrm{~g}, 0.205 \mathrm{meq} . \mathrm{g}, 0.018 \mathrm{mmol})$ were treated with the same reaction conditions and washing protocols described above for the transformation of $\mathbf{3 2} \rightarrow \mathbf{3 5}$. Solvent was then removed in vacuo to yield unreacted 43 as light yellow beads. $5.2 \mathrm{mg}$ of this resin was then treated with HF/Pyridine cleavage conditions (see General Methods) to yield crude alcohol $\mathbf{4 3}_{\mathrm{M}=\mathrm{H}}$ with LCMS purity $>90 \%\left(\square_{214}, \mathrm{t}_{\mathrm{R}} 9.55 \mathrm{~min}\right) .84 .2 \mathrm{mg}$ of this resin was then treated with $\mathrm{HF} /$ Pyridine cleavage conditions and the crude product was purified by flash chromatography $\left(\mathrm{SiO}_{2}\right.$, hexanes:EtOAc $\left.1: 1 \rightarrow 1: 2\right)$ to afford alcohol $\mathbf{4 3}_{\mathrm{M}=\mathrm{H}}$ as a yellow oil (8.4 $\mathrm{mg}, 0.053 \mathrm{mmol}, 0.181 \mathrm{meq} . / \mathrm{g}$, Theoretical yield $0.205 \mathrm{meq} . / \mathrm{g}, 88 \%$ from substrate 43 ).
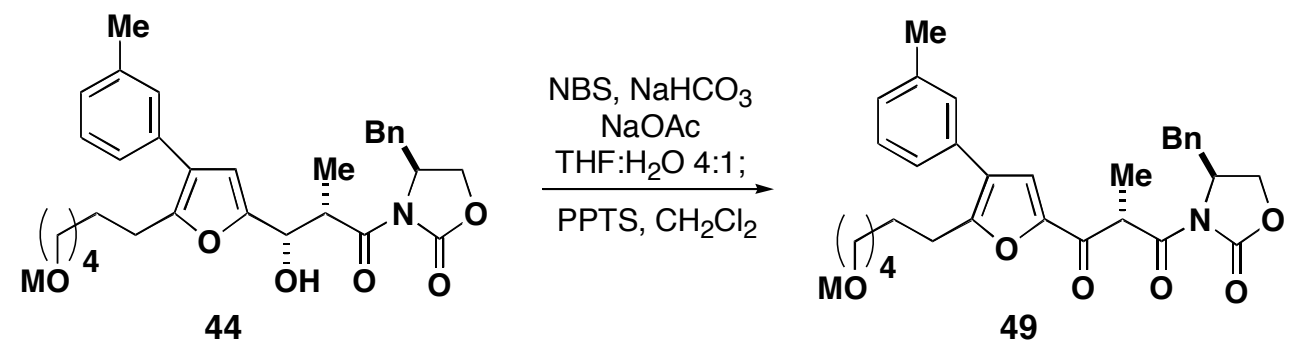

\section{Macrobead-bound-1-((4S)-4-Benzyl-2-oxo-oxazolidin-3-yl)-3-[5-(6-hydroxy-hexyl)-4-m- tolyl-furan-2-yl]-(2S)-2-methyl-propane-1,3-dione (49)}

Light yellow beads $44(0.090 \mathrm{~g}, 0.460 \mathrm{meq} . / \mathrm{g}, 0.041 \mathrm{mmol})$ were treated with the same reaction conditions and washing protocol described above for the transformation of $\mathbf{3 2} \rightarrow \mathbf{3 5}$. Solvent was then removed in vacuo to yield 49 as light yellow beads. $5.2 \mathrm{mg}$ of this resin was then treated with HF/Pyridine cleavage conditions (see General Methods) to yield crude alcohol $4_{\mathrm{M}=\mathrm{H}}$ with LCMS purity $72 \%\left(\square_{214}, \mathrm{t}_{\mathrm{R}} 10.12 \mathrm{~min}\right) .86 .1 \mathrm{mg}$ of this resin was then treated with $\mathrm{HF} /$ Pyridine cleavage conditions and the crude product was purified by flash chromatography $\left(\mathrm{SiO}_{2}\right.$, hexanes:EtOAc 2:1 $\rightarrow$ 1:2) to afford alcohol $\mathbf{4 9}_{\mathrm{M=H}}$ as a yellow oil (15.2 $\mathrm{mg}, 0.0294$ mmol, 0.341 meq./g, Theoretical yield 0.460 meq./g, 74\% from 44). TLC (hexanes:EtOAc 1:1) $\mathrm{R}_{\mathrm{f}}=0.24$; FTIR (film, $\mathrm{cm}^{-1}$ ) 3524, 2933, 2859, 1780, 1706, 1700, 1524, 1482, 1454, 1390, 1358, 1213, 1125, 1014; ${ }^{1} \mathrm{H}$ NMR $\left(500 \mathrm{MHz}, \mathrm{CDCl}_{3}\right) \square 7.36-7.13(\mathrm{~m}, 10 \mathrm{H}, \mathrm{ArH}, \mathrm{PhH}$, and FurH), 5.28 $\left(\mathrm{q}, J=7.5 \mathrm{~Hz}, 1 \mathrm{H}, \mathrm{CHCH}_{3}\right), 4.77\left(\mathrm{~m}, 1 \mathrm{H}, \mathrm{C}_{4}-\mathbf{H}\right), 4.25\left(\right.$ app t, $\left.J=8.5 \mathrm{~Hz}, 1 \mathrm{H}, \mathrm{C}_{5}-\mathbf{H H}\right), 4.18$ (dd, $\left.J=9,2.5 \mathrm{~Hz}, 1 \mathrm{H}, \mathrm{C}_{5}-\mathrm{HH}\right), 3.60\left(\mathrm{t}, J=6.5 \mathrm{~Hz}, 2 \mathrm{H}, \mathrm{CH}_{2} \mathrm{OH}\right), 3.37\left(\mathrm{dd}, J=13,3 \mathrm{~Hz}, 1 \mathrm{H}, \mathrm{C}_{4^{-}}\right.$ CHHPh), 2.84 (t, $\left.J=7.5 \mathrm{~Hz}, 2 \mathrm{H}, \mathrm{FurCH}_{2}\right), 2.79$ (dd, $\left.J=14,10 \mathrm{~Hz}, 1 \mathrm{H}, \mathrm{C}_{4}-\mathrm{CHHPh}\right), 2.39$ (s, $\left.3 \mathrm{H}, \mathrm{ArCH}_{3}\right), 1.76\left(\mathrm{~m}, 2 \mathrm{H}, \mathrm{FurCH}_{2} \mathrm{CH}_{2}\right), 1.58\left(\mathrm{~d}, J=7 \mathrm{~Hz}, 3 \mathrm{H}, \mathrm{CHCH}_{3}\right), 1.53(\mathrm{~m}, 2 \mathrm{H}$, $\mathrm{CH}_{2} \mathrm{CH}_{2} \mathrm{OH}$ ), 1.40-1.34 (m, 4H, $\left.\mathrm{CH}_{2} \mathrm{CH}_{2} \mathrm{CH}_{2} \mathrm{CH}_{2} \mathrm{OH}\right) ;{ }^{13} \mathrm{C}$ NMR (100 MHz, $\left.\mathrm{CDCl}_{3}\right) \square$ 185.7, $170.4,157.5,153.9,149.4,138.7,135.4$, 132.6, 129.7, 129.2, 129.0, 128.9, 128.4, 127.6, 125.2, 125.0, 120.1, 66.8, 63.0, 55.7, 49.1, 38.2, 32.7, 29.1, 28.1, 27.4, 25.5, 21.7, 14.0; HRMS (ES ${ }^{+}$ calculated for $\mathrm{C}_{31} \mathrm{H}_{35} \mathrm{NO}_{6}(\mathrm{M}+\mathrm{H})^{+}:$518.2542, found: 518.2532 . 


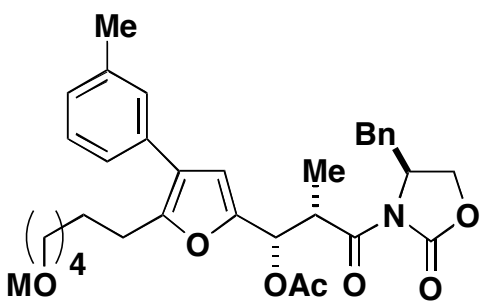

45

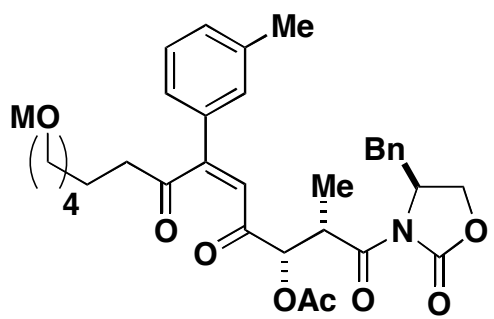

50

Macrobead-bound-acetic acid (1S)-1-[2-((4S)-4-benzyl-2-oxo-oxazolidin-3-yl)-(1S)-1methyl-2-oxo-ethyl]-11-hydroxy-2,5-dioxo-4-m-tolyl-undec-3-enyl ester (50)

Light yellow beads $45(0.090 \mathrm{~g}, 0.379 \mathrm{meq} . / \mathrm{g}, 0.034 \mathrm{mmol})$ were treated with the same reaction conditions and washing protocol described above for the transformation of $\mathbf{3 2} \rightarrow \mathbf{3 5}$. Solvent was then removed in vacuo to yield $\mathbf{5 0}$ as light yellow beads. $5.2 \mathrm{mg}$ of this resin was then treated with HF/Pyridine cleavage conditions (see General Methods) to yield crude alcohol $\mathbf{5 0}_{\mathrm{M}=\mathrm{H}}$ with LCMS purity $66 \%\left(\square_{214}, \mathrm{t}_{\mathrm{R}} 9.57 \mathrm{~min}\right) .84 .4 \mathrm{mg}$ of this resin was then treated with $\mathrm{HF} /$ Pyridine cleavage conditions and the crude product was purified by flash chromatography $\left(\mathrm{SiO}_{2}\right.$, hexanes:EtOAc $\left.2: 1 \rightarrow 1: 2\right)$ to afford alcohol $\mathbf{5 0}_{\mathrm{M}=\mathrm{H}}$ as a yellow oil (13.3 $\mathrm{mg}, 0.0230$ mmol, 0.273 meq./g, Theoretical yield 0.377 meq./g, 72\% from 45). TLC (hexanes:EtOAc 1:2) $\mathrm{R}_{\mathrm{f}}=0.29$; FTIR (film, $\mathrm{cm}^{-1}$ ) 3537, 2934, 2859, 1779, 1746, 1702, 1577, 1454, 1388, 1223, 1106, 1048; ${ }^{1} \mathrm{H}$ NMR $\left(500 \mathrm{MHz}, \mathrm{CDCl}_{3}\right)$ ] 7.35-7.24 (m, 7H, ArH and PhH), 7.20-7.17 (m, 2H, PhH), $6.81(\mathrm{~s}, 1 \mathrm{H}, \mathrm{CHCOCHOAc}), 5.74(\mathrm{~d}, J=5 \mathrm{~Hz}, 1 \mathrm{H}, \mathrm{CHOAc}), 4.62\left(\mathrm{~m}, 1 \mathrm{H}, \mathrm{C}_{4}-\mathbf{H}\right), 4.36-4.30$ (m, $2 \mathrm{H}, \mathrm{CHCH}_{3}$ and $\left.\mathrm{C}_{5}-\mathrm{HH}\right), 4.20\left(\mathrm{dd}, J=9,2 \mathrm{~Hz}, 1 \mathrm{H}, \mathrm{C}_{5}-\mathrm{HH}\right), 3.61\left(\mathrm{t}, J=7 \mathrm{~Hz}, 2 \mathrm{H}, \mathrm{CH}_{2} \mathrm{OH}\right), 3.25$ $\left(\mathrm{dd}, J=13.5,3 \mathrm{~Hz}, 1 \mathrm{H}, \mathrm{C}_{4}-\mathrm{CHHPh}\right), 2.80\left(\mathrm{dd}, J=13.5,9.5 \mathrm{~Hz}, 1 \mathrm{H}, \mathrm{C}_{4}-\mathrm{CHHPh}\right), 2.61(\mathrm{dt}, J=$ $\left.18,7 \mathrm{~Hz}, 1 \mathrm{H}, \mathrm{COCHHCH}_{2}\right), 2.51\left(\mathrm{dt}, J=18.5,7.5 \mathrm{~Hz}, 1 \mathrm{H}, \mathrm{COCHHCH}_{2}\right), 2.37(\mathrm{~s}, 3 \mathrm{H}, \mathrm{ArCH})$, $2.18\left(\mathrm{~s}, 3 \mathrm{H}, \mathrm{COCH}_{3}\right), 1.72\left(\mathrm{~m}, 2 \mathrm{H}, \mathrm{CH}_{2} \mathrm{CH}_{2} \mathrm{OH}\right), 1.54\left(\mathrm{~m}, 2 \mathrm{H}, \mathrm{COCH}_{2} \mathrm{CH}_{2}\right), 1.39-1.33(\mathrm{~m}, 4 \mathrm{H}$, $\left.\mathrm{CH}_{2} \mathrm{CH}_{2} \mathrm{CH}_{2} \mathrm{CH}_{2} \mathrm{OH}\right), 1.23$ (d, $\left.J=6.5 \mathrm{~Hz}, 3 \mathrm{H}, \mathrm{CHCH}_{3}\right) ;{ }^{13} \mathrm{C} \mathrm{NMR}\left(100 \mathrm{MHz}, \mathrm{CDCl}_{3}\right) \square 207.0$, 192.9, 172.4, 170.4, 159.5, 153.5, 139.0, 135.0, 132.9, 131.9, 129.4, 129.1, 129.0, 127.7, 127.4, 124.3, 117.9, 77.11, 66.6, 62.8, 55.6, 41.9, 39.2, 37.8, 32.5, 28.4, 25.3, 22.8, 21.4, 20.7, 11.2; HRMS $\left(\mathrm{ES}^{+}\right)$calculated for $\mathrm{C}_{33} \mathrm{H}_{39} \mathrm{NO}_{8}\left(\mathrm{M}+\mathrm{NH}_{4}\right)^{+}: 595.3019$, found: 595.3034 .

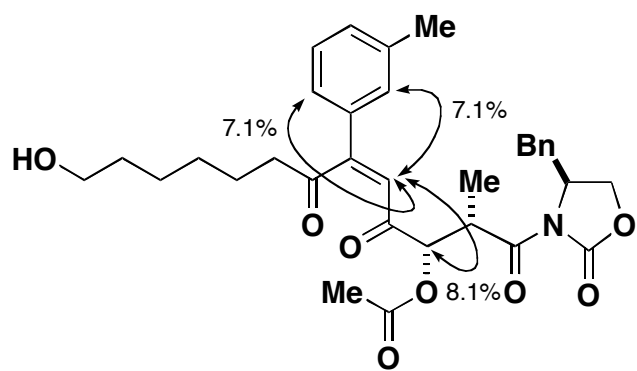

Selected nuclear Overhauser enhancements observed with a one-dimensional nOe experiment (irradiation of CHCOCHOAc) 


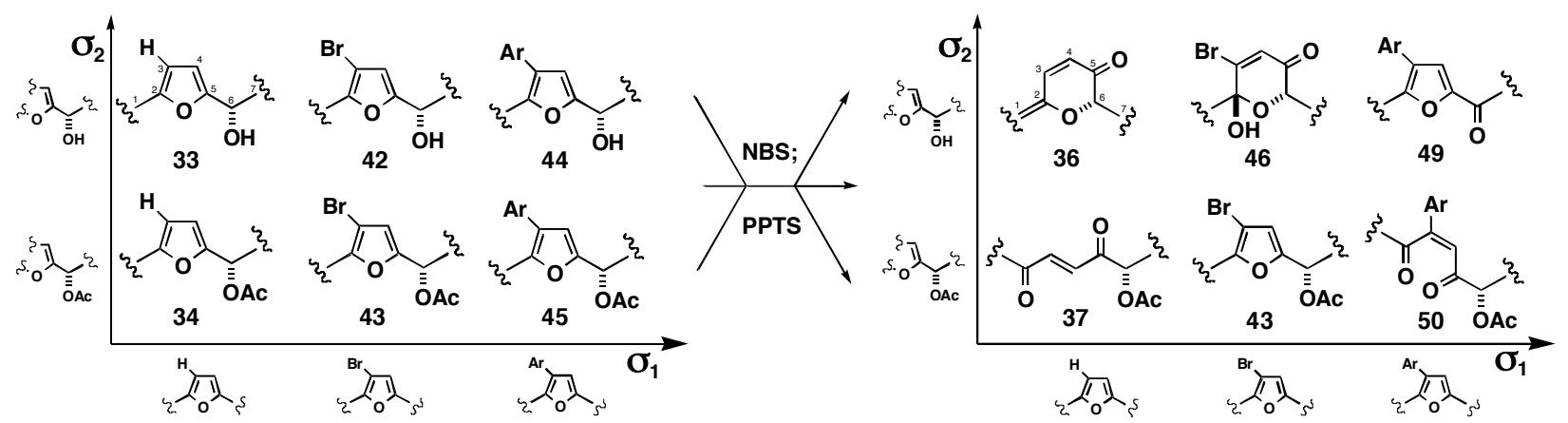

Figure 7A. (The following experiment was executed in triplicate) A single reaction vessel was charged with six individual macrobeads 33, 34, and 42-45 (representing a complete $3 \times 2$ matrix of $\square$-elements). To this mixture of macrobeads at $\mathrm{rt}$ under ambient was added THF:water 4:1 $(1.5 \mathrm{~mL}), \mathrm{NaHCO}_{3}(56.7 \mathrm{mg}, 0.675 \mathrm{mmol}), \mathrm{NaOAc}(27.7 \mathrm{mg}, 0.338 \mathrm{mmol})$, and $N$ bromosuccinimide $(40.0 \mathrm{mg}, 0.23 \mathrm{mmol})$. The resulting mixture was sealed, wrapped in aluminum foil, and tumbled at $\mathrm{rt}$ for $1 \mathrm{~h}$. The six macrobeads were then isolated from the reaction mixture by filtration and collectively washed as follows: $5 \times \mathrm{THF}, 5 \times \mathrm{H}_{2} \mathrm{O}, 5 \times \mathrm{THF}$, THF:water 3:1 x $1 \mathrm{~h}, 5$ x THF, THF x $1 \mathrm{~h}, 5 \times \mathrm{CH}_{2} \mathrm{Cl}_{2}, \mathrm{CH}_{2} \mathrm{Cl}_{2} \times 30 \mathrm{~min}, 5 \times$ anh. $\mathrm{CH}_{2} \mathrm{Cl}_{2}$, anh. $\mathrm{CH}_{2} \mathrm{Cl}_{2} \times 30 \mathrm{~min}$. After removing the solvent in vacuo, the six macrobeads were transferred collectively to a new reaction vessel containing a $0.00075 \mathrm{M}$ solution of pyridinium $p$ toluenesulfonate in $\mathrm{CH}_{2} \mathrm{Cl}_{2}(2 \mathrm{~mL})$. The resulting mixture was sealed under a cloud of argon and maintained at $40-45^{\circ} \mathrm{C}$ (oil bath) for $20 \mathrm{~h}$. The six macrobeads were then isolated from the reaction mixture by filtration and washed as follows: $5 \times$ THF, $5 \times \mathrm{H}_{2} \mathrm{O}, 5 \times$ THF, THF:dilute aq. $\mathrm{NaHCO}_{3}$ (sat. aq. $\mathrm{NaHCO}_{3}: \mathrm{H}_{2} \mathrm{O}$ 1:2) 1:1 x 1 h, 5 x THF, 5 x H $\mathrm{H}_{2} \mathrm{O}, 5$ x THF, THF:dilute aq. $\mathrm{NH}_{4} \mathrm{Cl}$ (sat. aq. $\mathrm{NH}_{4} \mathrm{Cl}: \mathrm{H}_{2} \mathrm{O}$ 1:2) 1:1 x $1 \mathrm{~h}, 5$ x THF, 5 x $\mathrm{H}_{2} \mathrm{O}, 5$ x THF, THF: $\mathrm{H}_{2} \mathrm{O}$ 3:1 x $1 \mathrm{~h}, 5$ x THF, THF x $1 \mathrm{~h}, 5 \times \mathrm{CH}_{2} \mathrm{Cl}_{2}, \mathrm{CH}_{2} \mathrm{Cl}_{2}$ x $30 \mathrm{~min}, 5 \mathrm{x}$ anh. $\mathrm{CH}_{2} \mathrm{Cl}_{2}$, anh. $\mathrm{CH}_{2} \mathrm{Cl}_{2}$ x $30 \mathrm{~min}$. Solvent was then removed in vacuo to yield six product macrobeads, which were segregated into individual polypropylene eppendorf tubes and treated with HF/Pyridine cleavage conditions (see General Methods). The cleaved products were then analyzed by LCMS: 6/6 of the anticipated compounds $\left(36_{\mathrm{M}=\mathrm{H}}, 37_{\mathrm{M}=\mathrm{H}}, 46_{\mathrm{M}=\mathrm{H}}, 43_{\mathrm{M}=\mathrm{H}}, 49_{\mathrm{M}=\mathrm{H}}\right.$, or $\left.50_{\mathrm{M}=\mathrm{H}}\right)$ were identified as the major product (by $\mathrm{t}_{\mathrm{R}}$ and mass) cleaved from an individual macrobead.

Figure 7B. The synthesis of diverse skeletons is critical to achieving diverse displays of chemical information in three-dimensional space. To provide some form of quantification for this type of diversity found in the set of six skeletons shown in Figure 7A, a skeletal diversity metric was developed based on the distance, angle, and dihedral angle between common atoms in computationally derived three-dimensional structures. Specifically, it was recognized that the missing bonds in both the substrates and products in Figure 7A represent potential attachment sites to which building blocks could be appended. The six substrates, having a $3 \times 2$ matrix of different appendages attached to a common $\square$-alkoxyalkyl furan skeleton resemble the types of compounds typically derived from the one synthesis-one skeleton approach. Alternatively, the six products represent six distinct molecular skeletons generated combinatorially using the $\square$ element-based synthesis strategy. Comparing and contrasting these two collections (which are almost constitutionally isomeric) can provide a metric for the skeletal diversity generated in this one reaction using a common set of reagents. By replacing each of the missing bonds in the 12 structures shown in Figure 7A with methyl groups (or a methylene group for the 'left side' of 
structure $\mathbf{3 6})$, a collection of 12 simplified structures were generated: six substrates $\left(\mathbf{3 3}^{*}, \mathbf{3 4}\right.$, $42^{*}, 43^{*}, 44^{*}$, and $\left.45^{*}\right)$ and six products $\left(36^{*}, 37^{*}, 46^{*}, 43^{*}, 49^{*}\right.$, and $\left.50^{*}\right)$, which all share in common the seven contiguous carbon atoms labeled $\mathrm{C}_{1}-\mathrm{C}_{7}$ (see below). Using the Spartan software package (Spartan '02, Wavefunction, Inc.) and a Gateway PC with an Intel Pentium 4 processor, the following two-step calculation was performed on all 12 structures: The equilibrium conformer was determined using the standard Spartan equilibrium conformer search with semiempirical AM1 calculations, followed by the determination of equilibrium geometry using the Hartree-Fock method with the 6-31G* split-valence basis set.

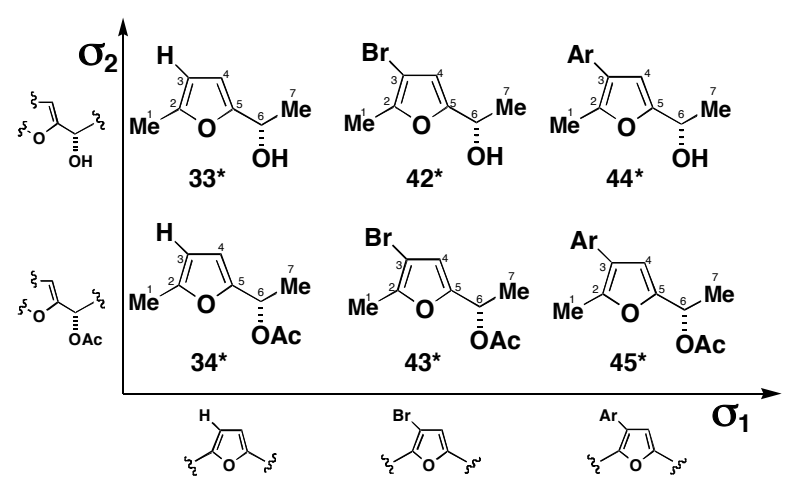

Substrates

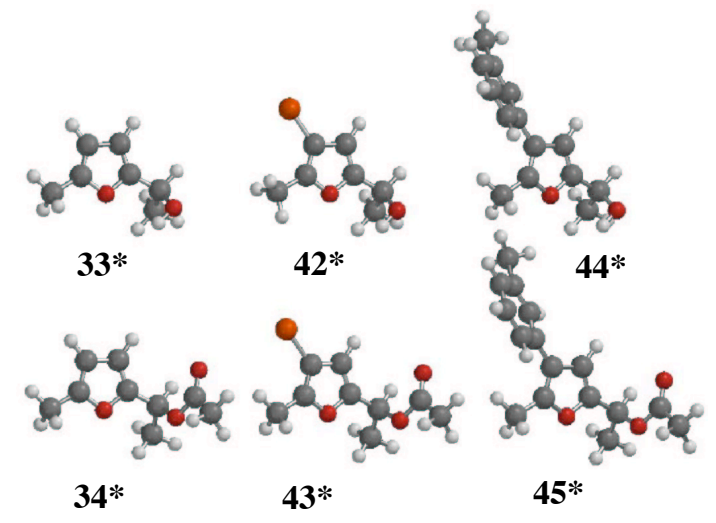

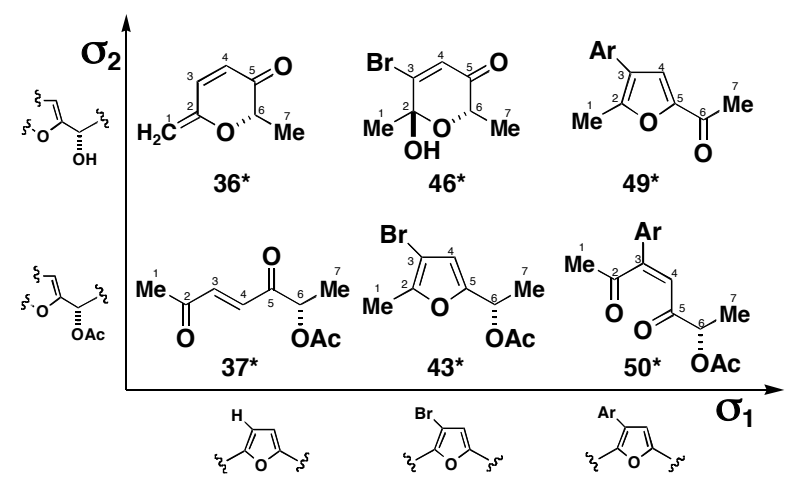

Products
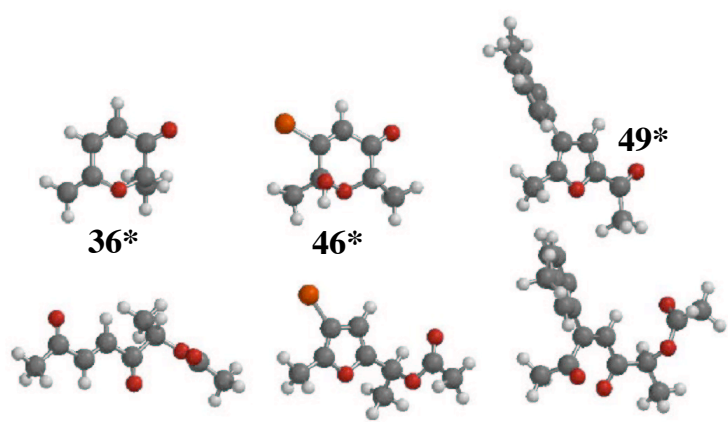

$37^{*}$

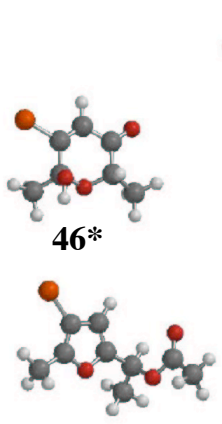

$47^{*}$
50*

For each of these 12 computationally derived three-dimensional structures, the positions of every other carbon in the common, contiguous seven-carbon atom stretch were then used to determine the following three parameters (each parameter provides unique information regarding the relative positions of the building block attachment sites, $\mathrm{C}_{1}$ and $\mathrm{C}_{7}$, in three-dimensional space:

1. the distance (in angstroms) between $\mathrm{C}_{1}$ and $\mathrm{C}_{7}$.

2. the angle $\mathrm{C}_{1}$ - the midpoint between $\mathrm{C}_{3}$ and $\mathrm{C}_{5}-\mathrm{C}_{7}$.

3. the dihedral angle comprising $\mathrm{C}_{1}, \mathrm{C}_{3}, \mathrm{C}_{5}$, and $\mathrm{C}_{7}$.

This analysis produced the data shown in the following Table: 
Burke, Berger, and Schreiber - Supporting Information Part A
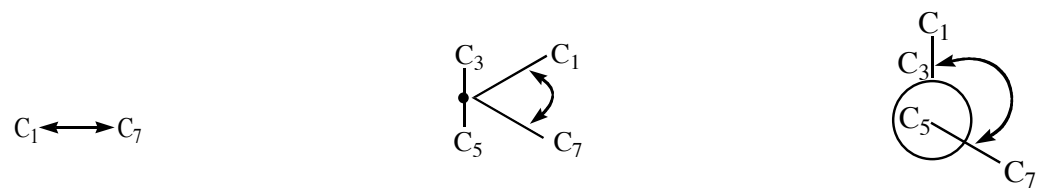

\begin{tabular}{|l|l|l|l|}
\hline Substrates & distance (angstroms) & angle (degrees) & dihedral angle (degrees) \\
\hline $\mathbf{3 3} *$ & 5.30 & 110.6 & 76.5 \\
\hline $\mathbf{3 4}$ & 5.17 & 105.9 & 56.8 \\
\hline $\mathbf{4 2}^{*}$ & 5.30 & 110.6 & 75.2 \\
\hline $\mathbf{4 3}^{*}$ & 5.16 & 105.8 & 55.7 \\
\hline $\mathbf{4 4}^{*}$ & 5.27 & 109.6 & 74.9 \\
\hline $\mathbf{4 5}^{*}$ & 5.14 & 104.9 & 54.2 \\
\hline standard deviation & $\mathbf{0 . 0 7}$ & $\mathbf{2 . 4}$ & $\mathbf{1 0 . 0}$ \\
\hline Products & & & \\
\hline $\mathbf{3 6} *$ & 4.04 & 83.7 & 39.4 \\
\hline $\mathbf{3 7}$ & 6.81 & 145.0 & 158.2 \\
\hline $\mathbf{4 6}^{*}$ & 4.64 & 91.0 & 14.9 \\
\hline $\mathbf{4 3}^{*}$ & 5.16 & 105.8 & 55.7 \\
\hline $\mathbf{4 9}^{\mathbf{5}}$ & 5.10 & 102.1 & 2.6 \\
\hline standard deviation & 5.90 & 123.1 & 128.5 \\
\hline & $\mathbf{0 . 8 9}$ & $\mathbf{2 0 . 5}$ & $\mathbf{5 7 . 5}$ \\
\hline
\end{tabular}

Plotting these parameters for both substrates and products in a three-dimensional plot using the Spotfire graphing package produced the three-dimensional plots shown in Figure 7B of the text. 


\section{Building block testing}

\section{Screening for Building Block \#1 (BB $)$}

The 13 commercially available compounds shown below, each containing both a hydroxyl group and a terminal olefin, were screened for both effective loading onto macrobeads and subsequent B-alkyl Suzuki coupling with one or more of the following: 5-bromofuraldehyde (28), 4,5-dibromofuraldehyde (38), and 4- $m$-MePh-5-bromofuraldehyde (53). All reactions were run on $\sim 25 \mathrm{mg}$ of macrobeads.

\section{Collection of potential building blocks included in screen for $\mathbf{B B}_{1}$}
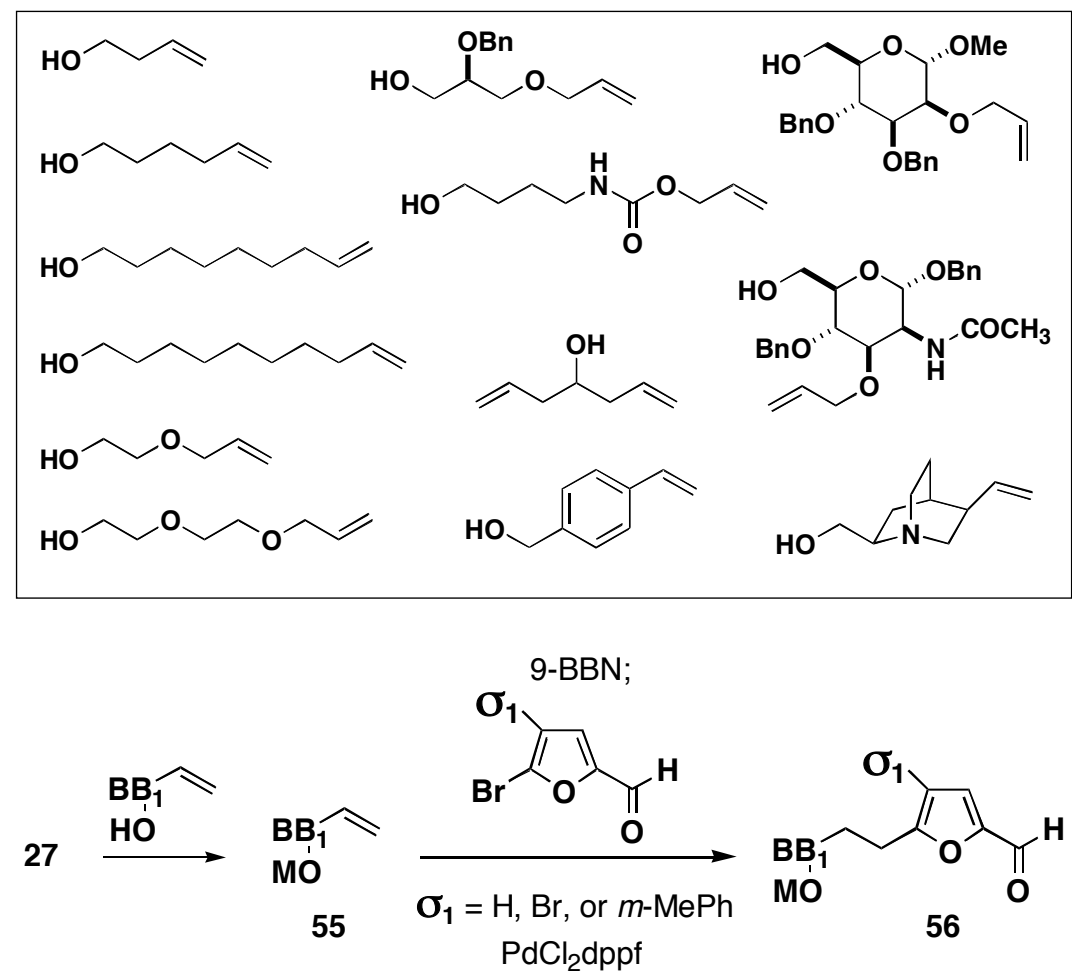

Screening for $\mathbf{B B}_{1}$ : Step 1. 3-[Diisopropyl( $p$-methoxyphenyl)silyl]propyl functionalized macrobeads ( $25 \mathrm{mg}$, estimated loading $\sim 1.3 \mathrm{meq} \mathrm{Si} / \mathrm{g}, \sim 0.0325 \mathrm{meq}$.) in a $2 \mathrm{~mL}$ polypropylene tube at $\mathrm{rt}$ under argon were allowed to swell in $\mathrm{CH}_{2} \mathrm{Cl}_{2}(\sim 10 \mathrm{ml})$ for $10 \mathrm{~min}$. The colorless beads were then filtered and again washed with $\mathrm{CH}_{2} \mathrm{Cl}_{2}(\sim 10 \mathrm{~mL}$ x $10 \mathrm{~min}$.), and then resuspended in a $2.5 \%(\mathrm{v} / \mathrm{v})$ solution of $\mathrm{TMSCl}$ in $\mathrm{CH}_{2} \mathrm{Cl}_{2}(\sim 10 \mathrm{~mL})$ for $30 \mathrm{~min}$. The beads were again filtered and washed thrice with $\mathrm{CH}_{2} \mathrm{Cl}_{2}(5 \mathrm{~min}$ each) and then suspended in a $3 \%(\mathrm{v} / \mathrm{v})$ solution of trifluoromethanesulfonic acid in $\mathrm{CH}_{2} \mathrm{Cl}_{2}(0.575 \mathrm{~mL}, 0.195 \mathrm{mmol})$ for 20 min during which the reaction tube was shaken periodically and the beads turned orange. After filtration, the orangecolored beads were again thrice washed with $\mathrm{CH}_{2} \mathrm{Cl}_{2}$ and then resuspended in a minimum volume of $\mathrm{CH}_{2} \mathrm{Cl}_{2}(\sim 0.2 \mathrm{~mL})$. Freshly distilled 2,6-lutidine was then added ( $\left.30.3 \mathrm{uL}, 0.26 \mathrm{mmol}\right)$ resulting in bead discoloration followed by building block \#1 $(0.26 \mathrm{mmol})$. The resulting colorless reaction mixture was then shaken manually and let stand at $\mathrm{rt}$ for $12 \mathrm{~h}$. The beads were then filtered, washed with $\mathrm{CH}_{2} \mathrm{Cl}_{2}(5 \times 5 \mathrm{~mL} \times 5 \mathrm{~min}$. each), and the solvent was removed under 
argon flow followed by residual solvent removal in vacuo to yield resin $\mathbf{5 5}$ loaded with candidates for building block \#1.

Screening for $\mathbf{B B}_{2}$ : Step 2. Macrobeads loaded with candidates for building block \#1 55 ( $\sim 0.0325$ meq.) were washed with THF $(2 \times 3 \mathrm{~mL} \times 10 \mathrm{~min}$ each $)$ at $\mathrm{rt}$ and then resuspended in THF $(0.750 \mathrm{~mL})$. A $0.5 \mathrm{M}$ solution of $9-\mathrm{BBN}$ in THF $(0.5 \mathrm{~mL}, 0.25 \mathrm{mmol})$ was then added and the resulting mixture was let stand at $\mathrm{rt}$ for $5 \mathrm{~h}$ (with periodic manual agitation every hour). The reaction solution was then removed via cannula and the colorless resin was washed thoroughly with THF ( 5 x $5 \mathrm{~mL}$ x $10 \mathrm{~min}$ each). To the resin was then added $\mathrm{PdCl}_{2} \mathrm{dppf}(1 \mathrm{mg}, 0.00125$ mmol) via cannula as a suspension in THF $(0.125 \mathrm{~mL})$, one of the following three furaldehyde coupling partners: 5-bromofuraldehyde $(21.9 \mathrm{mg}, 0.125 \mathrm{mmol}), 4,5$-dibromofuraldehyde (31.7 $\mathrm{mg}, 0.125 \mathrm{mmol})$, or 4- $m$-MePh-5-bromofuraldehyde (33.1 $\mathrm{mg}, 0.125 \mathrm{mmol})$ via cannula as a solution in THF $(0.188 \mathrm{~mL})$, a $2 \mathrm{M}$ solution of $\mathrm{NaOH}(31 \square \mathrm{L}, 0.0625 \mathrm{mmol})$. The resulting orange reaction mixture was sealed under a cloud of argon and heated at $60-65{ }^{\circ} \mathrm{C}$ with periodic manual agitation for 24-28 h (reaction mixture turned dark brown). The yellow/orange resin was then isolated by filtration and washed as follows, $4 \times\left(5 \times\right.$ THF, $5 \times \mathrm{H}_{2} \mathrm{O}, 5 \times$ THF, THF: $\mathrm{H}_{2} \mathrm{O} 3: 1$ x $30 \mathrm{~min}$ ), 5 x THF, THF x $30 \mathrm{~min}, 5 \times \mathrm{CH}_{2} \mathrm{Cl}_{2}, \mathrm{CH}_{2} \mathrm{Cl}_{2}$ x $30 \mathrm{~min}$, and the residual solvent was removed in vacuo to yield product resin 56. $5 \mathrm{mg}$ of this resin was then treated with $\mathrm{HF} / \mathrm{Pyridine}$ cleavage conditions (see General Methods) and the crude product residue was analyzed by ${ }^{1} \mathrm{H}$ NMR, LCMS, and, in some cases, HRMS.

This building block screen led to the identification of the seven building blocks shown below (abbreviated $\mathbf{B B}_{\mathbf{1}} \mathbf{A}, \mathbf{B B}_{\mathbf{1}} \mathbf{B}, \mathbf{B B}_{1} \mathbf{C}$, etc.).

\section{Building Block \#1 $\left(\mathrm{BB}_{1}\right)$}

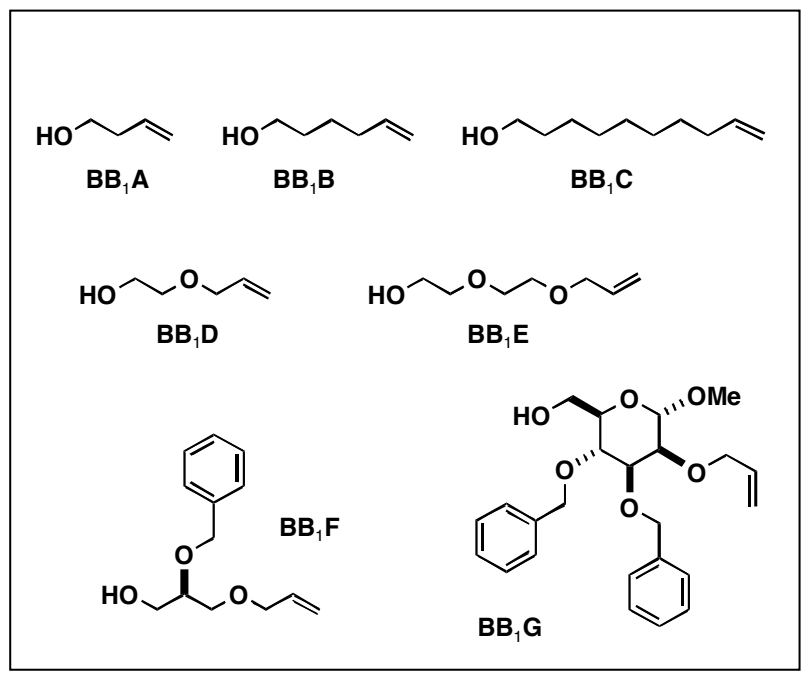

The results for these building blocks are shown below in tabular format: 
Burke, Berger, and Schreiber - Supporting Information Part A

Results for BB\#1: products $\mathbf{5 6}_{\mathrm{M}=\mathrm{H}}$

\begin{tabular}{|c|c|c|c|c|c|c|c|}
\hline $\mathbf{B B}_{1}$ & $\square_{1}$ & ${ }^{1} \mathrm{H}$ NMR & $\begin{array}{c}\text { \% Purity } \\
\text { LCMS, } \square_{280}\end{array}$ & $\begin{array}{c}\% \text { Purity } \\
\text { LCMS, } \square_{214}\end{array}$ & Ionization & $\begin{array}{c}\text { HRMS } \\
\text { Calculated }\end{array}$ & Observed \\
\hline $\mathbf{B B}_{1} \mathbf{A}$ & $\mathrm{H}$ & $\sqrt{ }$ & $>95$ & - & EI+ & $(\mathrm{m} / \mathrm{z}) 168.0786$ & 168.0785 \\
\hline $\mathbf{B B}_{1} \mathbf{B}$ & $\mathrm{H}$ & $\sqrt{ }$ & $>85$ & - & ES+ & $\left(\mathrm{M}+\mathrm{H}^{+}\right) 197.1177$ & 197.1177 \\
\hline $\mathrm{BB}_{1} \mathrm{C}$ & $\mathrm{H}$ & $\sqrt{ }$ & $>95$ & - & EI+ & $(\mathrm{m} / \mathrm{z}) 252.1725$ & 252.1723 \\
\hline $\mathbf{B B}_{1} \mathbf{D}$ & $\mathrm{H}$ & $\sqrt{ }$ & $>95$ & - & EI+ & $(\mathrm{m} / \mathrm{z}) 198.0892$ & 198.0893 \\
\hline $\mathbf{B B}_{1} \mathbf{E}$ & $\mathrm{H}$ & $\sqrt{ }$ & $>95$ & - & EI+ & $(\mathrm{m} / \mathrm{z}) 242.1154$ & 242.1152 \\
\hline $\mathbf{B B}_{1} \mathbf{F}$ & $\mathrm{H}$ & $\sqrt{ }$ & 93 & - & EI+ & $(\mathrm{m} / \mathrm{z}) 318.1467$ & 318.1464 \\
\hline $\mathbf{B B}_{1} \mathbf{G}$ & $\mathrm{H}$ & $\sqrt{ }$ & $>95$ & - & ES+ & $\left(\mathrm{M}+\mathrm{Na}^{+}\right) 533.2151$ & 533.2145 \\
\hline $\mathbf{B B}_{1} \mathbf{A}$ & $\mathrm{Br}$ & $\sqrt{ }$ & $>95$ & - & ES+ & $\left(\mathrm{M}+\mathrm{H}^{+}\right) 246.9970$ & 246.9969 \\
\hline $\mathbf{B B}_{1} \mathbf{B}$ & $\mathrm{Br}$ & $\sqrt{ }$ & - & $>95$ & ES+ & $\left(\mathrm{M}+\mathrm{H}^{+}\right) 397.0650$ & 397.0645 \\
\hline $\mathrm{BB}_{1} \mathrm{C}$ & $\mathrm{Br}$ & $\sqrt{ }$ & $>90$ & - & $\mathrm{ES}+$ & $\left(\mathrm{M}+\mathrm{H}^{+}\right) 331.0909$ & 331.0906 \\
\hline $\mathbf{B B}_{1} \mathbf{D}$ & $\mathrm{Br}$ & $\sqrt{ }$ & $>95$ & - & ES+ & $\left(\mathrm{M}+\mathrm{H}^{+}\right) 277.0075$ & 277.0066 \\
\hline $\mathbf{B B}_{1} \mathbf{E}$ & $\mathrm{Br}$ & $\sqrt{ }$ & $>95$ & - & ES+ & $\left(\mathrm{M}+\mathrm{H}^{+}\right) 321.0337$ & 321.0326 \\
\hline $\mathbf{B B}_{1} \mathbf{F}$ & $\mathrm{Br}$ & $\sqrt{ }$ & $>95$ & - & $\mathrm{ES}+$ & $\left(\mathrm{M}+\mathrm{H}^{+}\right) 397.0650$ & 387.0643 \\
\hline $\mathbf{B B}_{1} \mathbf{G}$ & $\mathrm{Br}$ & $\sqrt{ }$ & 84 & - & $\mathrm{ES}+$ & $\left(\mathrm{M}+\mathrm{Na}^{+}\right) 611.1256$ & 611.1246 \\
\hline $\mathbf{B B}_{1} \mathbf{A}$ & $m-\mathrm{MeAr}$ & $\sqrt{ }$ & - & 90 & ES+ & $\left(\mathrm{M}+\mathrm{H}^{+}\right) 259.1134$ & 259.1340 \\
\hline $\mathbf{B B}_{1} \mathbf{B}$ & $m-\mathrm{MeAr}$ & $\sqrt{ }$ & - & $>95$ & ES+ & $\left(\mathrm{M}+\mathrm{H}^{+}\right) 287.1647$ & 287.1647 \\
\hline $\mathrm{BB}_{1} \mathrm{C}$ & $m-\mathrm{MeAr}$ & $\sqrt{ }$ & - & $>95$ & ES+ & $\left(\mathrm{M}+\mathrm{H}^{+}\right) 343.2273$ & 343.2272 \\
\hline $\mathbf{B B}_{1} \mathbf{D}$ & $m-\mathrm{MeAr}$ & $\sqrt{ }$ & - & 92 & ES+ & $\left(\mathrm{M}+\mathrm{H}^{+}\right) 289.1440$ & 289.1430 \\
\hline $\mathbf{B B}_{1} \mathbf{E}$ & $m-\mathrm{MeAr}$ & $\sqrt{ }$ & - & 89 & ES+ & $\left(\mathrm{M}+\mathrm{H}^{+}\right) 333.1702$ & 333.1709 \\
\hline $\mathbf{B B}_{1} \mathbf{F}$ & $m-\mathrm{MeAr}$ & $\sqrt{ }$ & - & 83 & ES+ & $\left(\mathrm{M}+\mathrm{H}^{+}\right) 409.2015$ & 409.2009 \\
\hline $\mathbf{B B}_{1} \mathbf{G}$ & $m-\mathrm{MeAr}$ & $\sqrt{ }$ & - & 80 & ES+ & $\left(\mathrm{M}+\mathrm{Na}^{+}\right) 623.2621$ & 623.2615 \\
\hline
\end{tabular}




\section{Screening for Building Block \#2 (BB $)$}

A variety of commercially available, nonracemic chiral oxazolidinones were combined with diverse acyl side chains and the resulting candidate building blocks were screened for efficient coupling with macrobead-bound 5-(6-hydroxyhexyl)-2-furaldehyde (30).

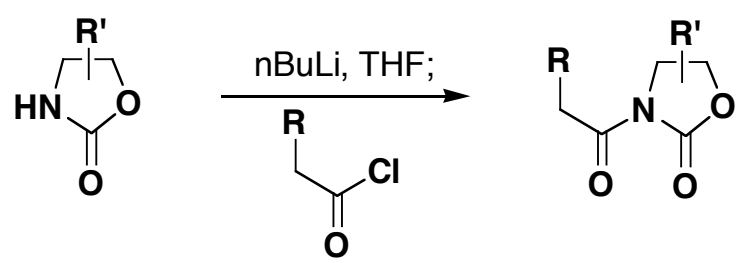

Synthesis of acyl oxazolidinones. A stirred solution of oxazolidinone $(1 \mathrm{~g})$ in anhydrous THF (0.2 $\mathrm{M}$ in oxazolidinone) was cooled to $-78{ }^{\circ} \mathrm{C}$. $n$-BuLi (1.1 equiv.) was slowly added and the mixture was stirred for 15 minutes. The appropriate acid chloride (1.1 equiv.) was then added by syringe and the mixture was stirred for another 30 minutes. The mixture was then warmed to rt over 45 minutes, quenched with $\mathrm{NH}_{4} \mathrm{Cl}(4 \mathrm{~mL})$, and the $\mathrm{THF}$ was removed with rotary evaporation. The resulting slurry was then extracted with $\mathrm{CH}_{2} \mathrm{Cl}(2 \times 5 \mathrm{~mL})$, and the combined organic fractions were washed with $2 \mathrm{M}$ aq. $\mathrm{NaOH}(5 \mathrm{~mL})$ and brine $(5 \mathrm{~mL})$, dried over sodium sulfate, and concentrated in vacuo. The product was then purified via flash chromatography $\left(\mathrm{SiO}_{2}\right.$, hexanes:EtOAc), azeotropically dried with benzene, and stored under argon. The average chemical yield of acyl oxazolidinone was roughly $85 \%$.

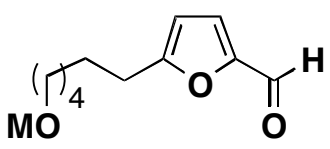

30

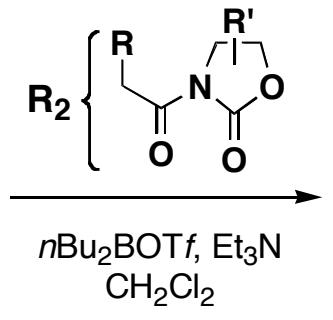

$\mathrm{CH}_{2} \mathrm{Cl}_{2}$

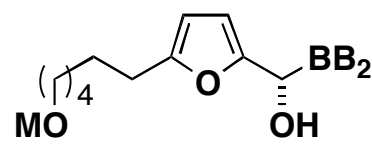

60

Screening of acyl oxazolidinones Yellow-orange macrobeads 30 (25 mg) were washed with $\mathrm{CH}_{2} \mathrm{Cl}_{2}(3 \times 1 \mathrm{~mL} \times 10 \mathrm{~min}$ each $)$ at $\mathrm{rt}$, and then cooled to $-78{ }^{\circ} \mathrm{C}$. In a separate vessel, to a stirred solution of acyl oxazolidinone $(0.125 \mathrm{mmol})$ in $\mathrm{CH}_{2} \mathrm{Cl}_{2}(0.5 \mathrm{~mL})$ at $0{ }^{\circ} \mathrm{C}$ was added a $1 \mathrm{M}$ solution of dibutylboron triflate in $\mathrm{CH}_{2} \mathrm{Cl}_{2}$ (131 $\left.\square \mathrm{L}, 0.131 \mathrm{mmol}\right)$ followed by triethylamine (21 $\square \mathrm{L}, 0.150 \mathrm{mmol})$. The resulting enolate solution was cooled to $-78{ }^{\circ} \mathrm{C}$ and then transferred rapidly via cannula to the vessel containing 30. The resulting mixture was sealed under a cloud of argon and maintained at $-78{ }^{\circ} \mathrm{C}$ for $48 \mathrm{~h},-26{ }^{\circ} \mathrm{C}$ for $24 \mathrm{~h}$, and $0{ }^{\circ} \mathrm{C}$ for $1 \mathrm{~h}$ (with periodic manual agitation about once every $8 \mathrm{~h}$ ). The reaction was then quenched with the addition of pH7 phosphate buffer (500 $\square \mathrm{L}), \mathrm{MeOH}(500 \square \mathrm{L})$, and $30 \%$ aq. $\mathrm{H}_{2} \mathrm{O}_{2}(333 \square \mathrm{L})$, and the resulting mixture was tumbled at $4{ }^{\circ} \mathrm{C}$ for $12-15 \mathrm{~h}$. Resin was then isolated by filtration and washed as follows: $5 \times \mathrm{CH}_{2} \mathrm{Cl}_{2}, 5 \times \mathrm{DMF}, 5 \times \mathrm{THF}, 5 \times \mathrm{CH}_{2} \mathrm{Cl}_{2}, \mathrm{CH}_{2} \mathrm{Cl}_{2} \times 1 \mathrm{~h}, 5 \times \mathrm{DMF}$, DMF x $1 \mathrm{~h}, 5 \mathrm{x}$ THF, THF x $1 \mathrm{~h}, 5 \times \mathrm{CH}_{2} \mathrm{Cl}_{2}, \mathrm{CH}_{2} \mathrm{Cl}_{2} \times 30 \mathrm{~min}, 5 \mathrm{x}$ anhydrous $\mathrm{CH}_{2} \mathrm{Cl}_{2}$, anhydrous $\mathrm{CH}_{2} \mathrm{Cl}_{2} \times 30$ min, and residual solvent was removed in vacuo to yield yellow product resin 60. $5 \mathrm{mg}$ of this 
product resin was then treated with HF/Pyridine cleavage conditions (see General Methods), and the crude product residue was analyzed by ${ }^{1} \mathrm{H}$ NMR and LCMS \pm HRMS.

Specifically, in the first of two rounds of screening, the diverse set of eight acyl oxazolidinones iviii shown below were synthesized and tested for efficient aldol coupling. Candidates $\mathbf{i}$ and vi were found to undergo efficient coupling with quantitative conversion and >10:1 d.r.

\section{Collection of candidate building blocks included in the first screen for $\mathbf{B B}_{2}$}

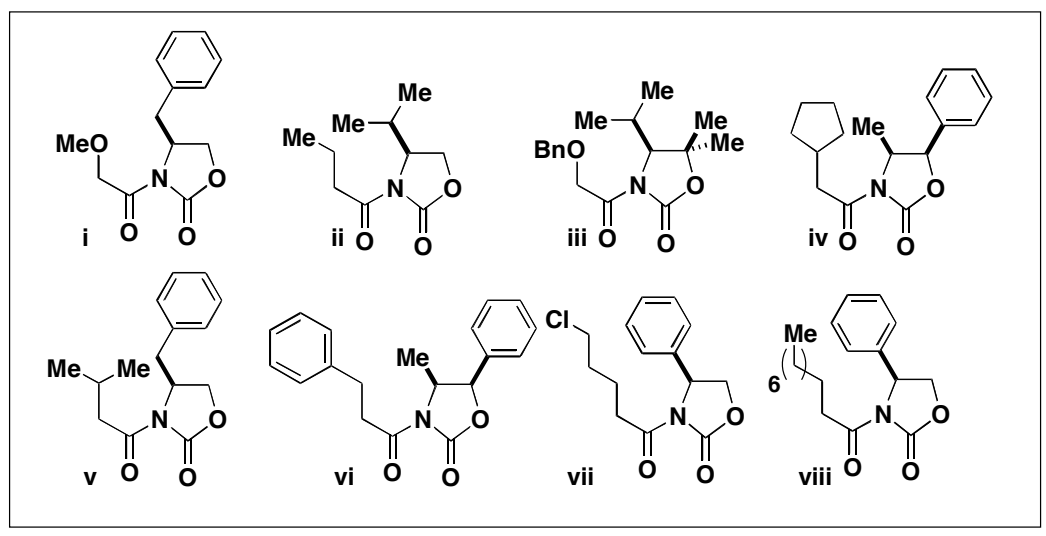

Based on the results of this first screen, the $5 \times 3$ matrix of commercially available oxazolidinones and acyl side chains $\mathbf{B B}_{2} \mathbf{A} \boldsymbol{S}-\mathbf{B B}_{2} \mathbf{O S}$ (shown below) were synthesized and tested for efficient aldol coupling with 5-(6-hydroxyhexyl)-2-furaldehyde (30). It was determined that all 15 of these candidate building blocks were effective coupling partners. The 15 enantiomeric acyl oxazolidinones ( $\mathbf{B B}_{\mathbf{2}} \mathbf{A} \boldsymbol{R}-\mathbf{B B}_{\mathbf{2}} \mathbf{O R}$, not shown) were also prepared, permitting access to both sets of possible enantiomeric and diastereomeric (when $\mathbf{B B}_{\mathbf{1}}$ is chiral) aldol adducts via reagentbased stereocontrol. These building blocks are classified as $R$ or $S$ by the orientation of the 4'substituent on the oxazolidinone ring.

\section{Collection of candidate building blocks included in the second screen for $\mathbf{B B}_{2}$}

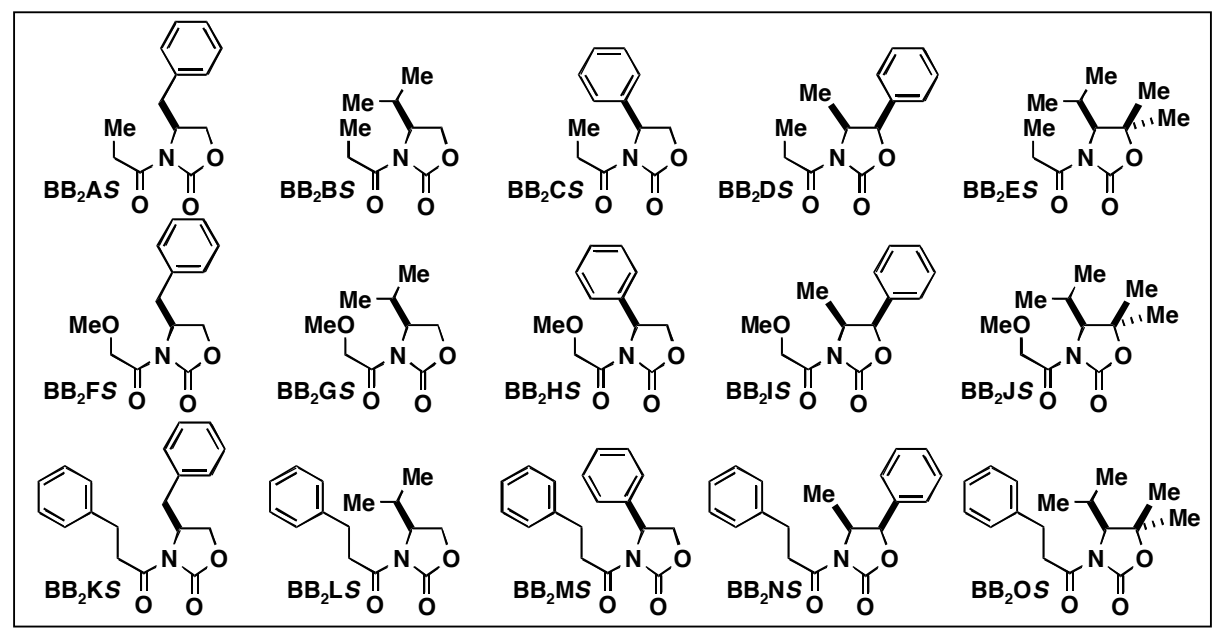


Burke, Berger, and Schreiber - Supporting Information Part A

The results of this second screen for $\mathrm{BB}_{2}$ are summarized intabular format below:

Results for BB\#2: products $60_{\mathrm{M}=\mathrm{H}}$

\begin{tabular}{|c|c|c|c|c|c|c|}
\hline & $\%$ Conversion & d.r. & $\%$ Purity & & HRMS & \\
\hline BB2 & ${ }^{1}$ H NMR & ${ }^{1} \mathrm{H}$ NMR & LCMS, $\square_{214}$ & Ionization & Calculated & Found \\
\hline $\mathrm{BB}_{2} \mathrm{~A} S$ & $>95$ & $20: 1$ & 86 & ES+ & $\left(\mathrm{M}+\mathrm{NH}_{4}^{+}\right) 447.2495$ & 447.2497 \\
\hline $\mathrm{BB}_{2} \mathrm{BS}$ & $>95$ & 20:1 & 92 & ES+ & $\left(\mathrm{M}+\mathrm{Na}^{+}\right) 404.2049$ & 404.2049 \\
\hline $\mathrm{BB}_{2} \mathrm{CS}$ & $>95$ & $20: 1$ & 91 & ES+ & $\left(\mathrm{M}+\mathrm{Na}^{+}\right) 438.1893$ & 438.1899 \\
\hline $\mathrm{BB}_{2} \mathrm{DS}$ & $>95$ & $20: 1$ & $>95$ & ES+ & $\left(\mathrm{M}+\mathrm{Na}^{+}\right) 452.2049$ & 452.2058 \\
\hline $\mathrm{BB}_{2} \mathrm{E} S$ & $>90$ & $8: 1$ & $>95$ & ES+ & $\left(\mathrm{M}+\mathrm{NH}_{4}^{+}\right) 427.2808$ & 427.2805 \\
\hline $\mathrm{BB}_{2} \mathrm{FS}$ & $>95$ & $10: 1$ & 92 & ES+ & $\left(\mathrm{M}+\mathrm{Na}^{+}\right) 468.1998$ & 468.2001 \\
\hline $\mathrm{BB}_{2} \mathrm{G} S$ & $>95$ & $12: 1$ & 86 & ES+ & $\left(\mathrm{M}+\mathrm{Na}^{+}\right) 420.1998$ & 420.2002 \\
\hline $\mathbf{B B}_{2} \mathbf{H} S$ & $>95$ & 11:1 & 83 & ES+ & $\left(\mathrm{M}+\mathrm{Na}^{+}\right) 454.1842$ & 454.1857 \\
\hline $\mathrm{BB}_{2} \mathrm{I} S$ & $>95$ & 16:1 & 77 & ES+ & $\left(\mathrm{M}+\mathrm{Na}^{+}\right) 468.1998$ & 468.1985 \\
\hline $\mathrm{BB}_{2} \mathrm{~J} S$ & $>95$ & 20:1 & 97 & ES+ & $\left(\mathrm{M}+\mathrm{Na}^{+}\right) 448.2311$ & 448.2314 \\
\hline $\mathrm{BB}_{2} \mathrm{~K} S$ & $>95$ & 20:1 & 97 & ES+ & $\left(\mathrm{M}+\mathrm{Na}^{+}\right) 528.2362$ & 528.2366 \\
\hline $\mathbf{B B}_{2} \mathbf{L} S$ & $>95$ & 10:1 & 72 & ES+ & $\left(\mathrm{M}+\mathrm{Na}^{+}\right) 480.2362$ & 480.2371 \\
\hline $\mathrm{BB}_{2} \mathrm{MS}$ & $>85$ & $7: 1$ & 90 & ES+ & $\left(\mathrm{M}+\mathrm{Na}^{+}\right) 514.2206$ & 514.2219 \\
\hline $\mathrm{BB}_{2} \mathrm{~N} S$ & $>90$ & $9: 1$ & 93 & ES+ & $\left(\mathrm{M}+\mathrm{NH}_{4}^{+}\right) 523.2808$ & 523.2816 \\
\hline $\mathrm{BB}_{2} \mathrm{OS}$ & $>85$ & $9: 1$ & 96 & ES+ & $\left(\mathrm{M}+\mathrm{Na}^{+}\right) 508.2675$ & 508.2670 \\
\hline
\end{tabular}




\section{Parallel synthesis}

To determine if the $(3 \times 2)$ combinatorial matrix of $\square$-elements shown in Figure 6 could effectively pre-encode the same matrix of six distinct skeletal outcomes when a complete, combinatorial matrix of building blocks was also attached to the same common core, 36 substrates 61a-jj were synthesized and exposed to common conditions in parallel as described below.

\section{Step 1. Loading of Building Block \#1 (BB $)$}

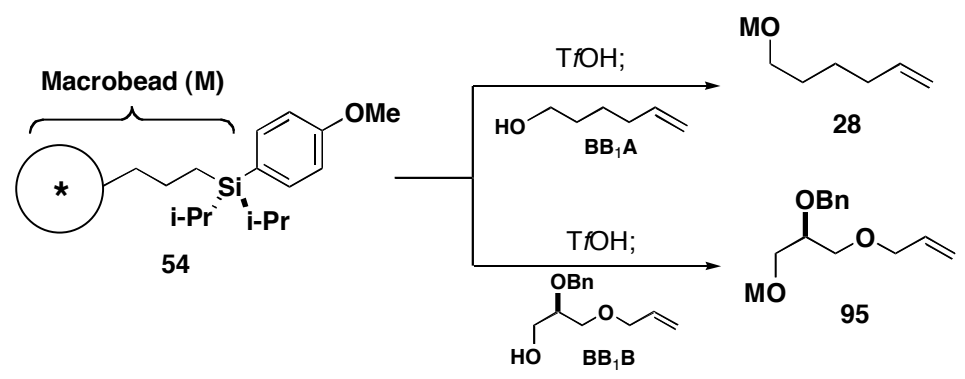

$1.2 \mathrm{~g}$ of 3-[Diisopropyl(p-methoxyphenyl)silyl]propyl functionalized macrobeads $\mathbf{5 4}$ was split into two portions (600 $\mathrm{mg}$ each) and each portion was subjected to a unique loading reaction with $\mathbf{B B}_{1} \mathbf{A}$ or $\mathbf{B B}_{\mathbf{1}} \mathbf{F}$, using the same protocol described previously for the transformation of $\mathbf{5 4}$ $\rightarrow \mathbf{2 8}$ to yield $\mathbf{2 8}$ and $\mathbf{9 5}$, which were carried on to step 2 .

Step 2. Suzuki coupling of Skeletal Information Element \#1 $\left(\square_{1}\right)$
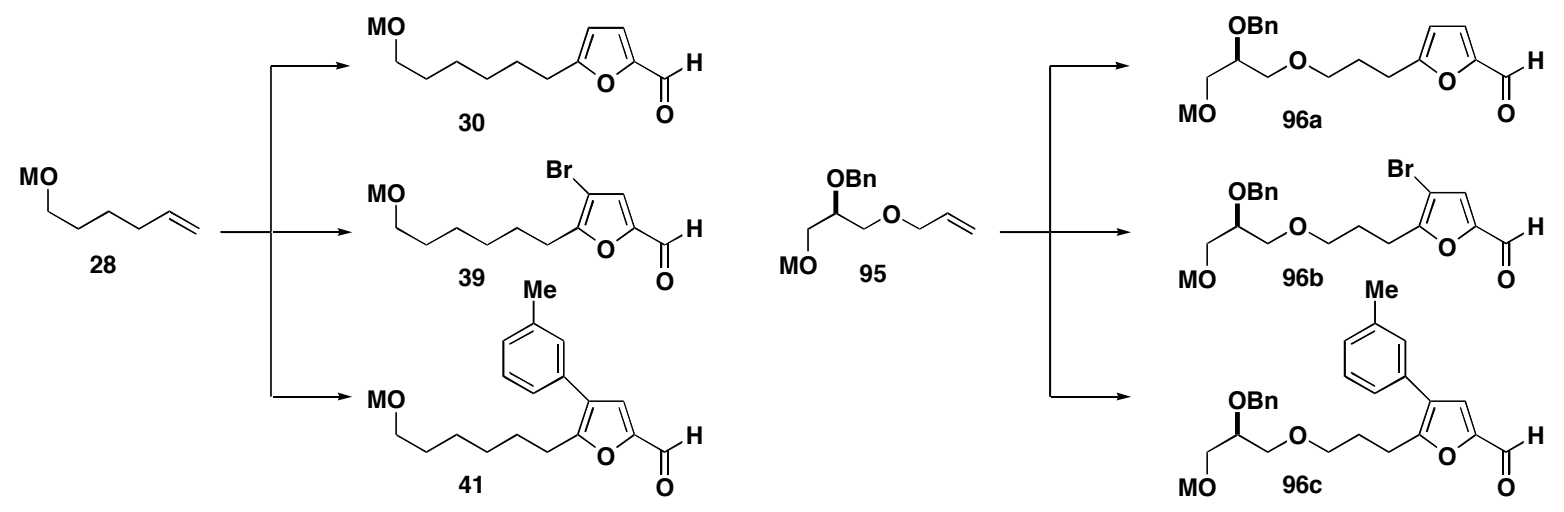

Suzuki coupling of Skeletal Information Element \#1 $\left(\square_{1}\right)$ Colorless beads $28(555 \mathrm{mg})$ and 95 (630 mg) were each split evenly by weight into three portions. Each of the three portions of 28 and 95 was then subjected to a B-alkyl Suzuki coupling with a unique 4-substituted-5bromofuraldehyde $\left(\square_{1}=\mathrm{H}, \square_{2}=\mathrm{Br}\right.$, or $\square_{3}=m$-MePh, six parallel reactions $)$ using the same protocols described previously for the transformation of $28 \rightarrow 30,28 \rightarrow 39$, and $28 \rightarrow 41$. The results are presented in tabular format below. 
Results of Step 2

\% Purity

$\begin{array}{cccccccccc}\text { Compound } & \mathbf{B B}_{\mathbf{1}} & \mathrm{Z}_{1} & \mathbf{B B}_{\mathbf{2}} & \mathrm{Z}_{2} & { }^{1} \mathbf{H} \mathbf{N M R} & \mathbf{L C M S}, \mathbf{2 1 4} \mathbf{~ n m} & \text { Ionization } & \text { Calculated } & \text { Observed } \\ \mathbf{3 0} & \mathrm{A} & \mathrm{H} & - & - & \sqrt{ } & >85 & \mathrm{ES}+ & \left(\mathrm{M}+\mathrm{H}^{+}\right) 197.1177 & 197.1177 \\ \mathbf{3 9} & \mathrm{A} & \mathrm{Br} & - & - & \sqrt{ } & >90 & \mathrm{ES}+ & \left(\mathrm{M}+\mathrm{H}^{+}\right) 275.0283 & 275.0282 \\ \mathbf{4 1} & \mathrm{A} & m-\mathrm{MePh} & - & - & \sqrt{ } & >90 & \mathrm{ES}+ & \left(\mathrm{M}+\mathrm{H}^{+}\right) 287.1647 & 287.1647 \\ \mathbf{9 6 a} & \mathrm{B} & \mathrm{H} & - & - & \sqrt{ } & 88(280 \mathrm{~nm}, 92) & \mathrm{ES}+ & \left(\mathrm{M}+\mathrm{H}^{+}\right) 319.1545 & 319.1536 \\ \mathbf{9 6 b} & \mathrm{B} & \mathrm{Br} & - & - & \sqrt{ } & 71(280 \mathrm{~nm}, 94) & \mathrm{ES}+ & \left(\mathrm{M}+\mathrm{H}^{+}\right) 397.0650 & 397.0645 \\ \mathbf{9 6 c} & \mathrm{B} & m-\mathrm{MePh} & - & - & \sqrt{ } & >90 & \mathrm{ES}+ & \left(\mathrm{M}+\mathrm{H}^{+}\right) 409.2015 & 409.2015\end{array}$

$\sqrt{ }: \quad{ }^{1} \mathrm{H}$ NMR spectrum consistent with anticipated structure, LCMS purities for $96 \mathbf{a}$ and $\mathbf{9 6} \mathbf{b}$ are reported with detection at both $\square_{214}$ and $\square_{280}$; all other LCMS data are reported with detection at $\square_{214}$.

\section{Step 3. Evans aldol coupling of Building Block \#2 $\left(\mathrm{BB}_{2}\right)$}
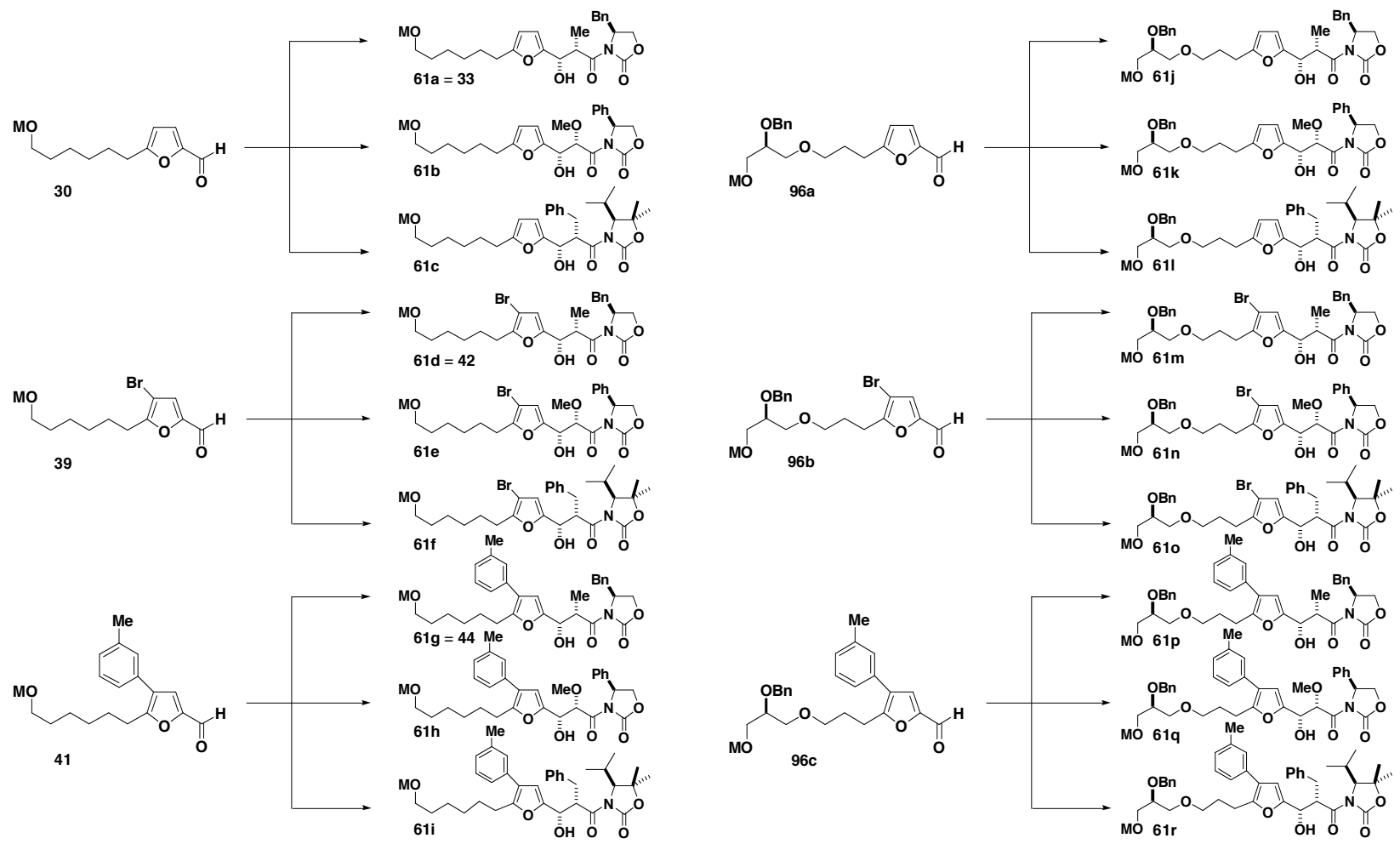

Aldol coupling of Building Block \#2 (BB $\mathbf{B}_{2}$. The six pools of light yellow resin from Step 2 $(30,39,41$, and 96a-c) were then each split into 3 equal portions (18 pools of $\sim 60 \mathrm{mg}$ each). Each of these 18 portions was then subjected to an aldol coupling reaction with one of the three acyl oxazolidinones $\mathbf{B B}_{2} \mathbf{A S}, \mathbf{B B}_{2} \mathbf{H S}$, or $\mathbf{B B}_{2} \mathbf{O S}$. Specifically, in 18 parallel reactions, 30, 39, 41, and 96a-c were transformed into 61a-r using the same protocol described previously for the transformation of $30 \rightarrow 33$. For the transformation of 96a-c $\rightarrow$ 611, 61o, and 61r, reactions were maintained at $-78{ }^{\circ} \mathrm{C}$ for $72 \mathrm{~h},-26{ }^{\circ} \mathrm{C}$ for $28 \mathrm{~h}$, and $0{ }^{\circ} \mathrm{C}$ for $2 \mathrm{~h}$ to promote full conversion. 
Step 4. Coupling of Skeletal Information Element \#2 $\left(\square_{2}\right)$
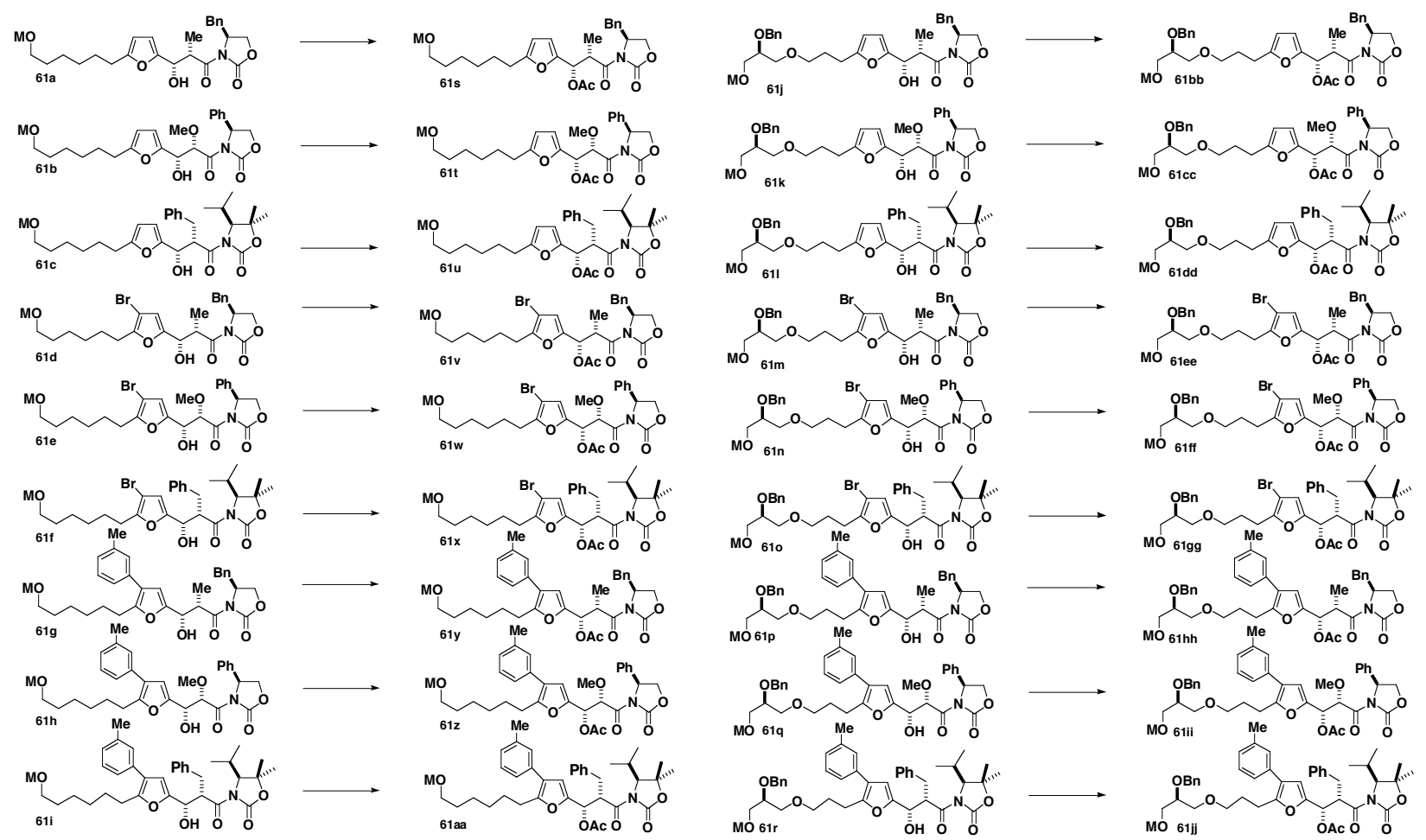

Step 4. Coupling of Skeletal Information Element \#2 $\left(\square_{2}\right): \pm$ Acetylation. 18 portions of light yellow resin from Step 3 (61a-r, $\sim 60 \mathrm{mg}$ each) were then each divided into two equal portions; one of these portions was subjected to an acetylation reaction using the same protocol described previously for the transformation of $\mathbf{3 3} \rightarrow \mathbf{3 4}$ and the other portion was not acetylated. This resulted in the net formation of the collection of 36 compounds $\mathbf{6 1 a} \mathbf{a} \mathbf{j j}$.

The results for Steps 3 and 4 are presented in tabular format below. 
Results of Steps 3 \& 4

\begin{tabular}{|c|c|c|c|c|c|c|c|c|c|}
\hline Compound & $\mathbf{B B}_{1}$ & $\square_{1}$ & $\mathbf{B B}_{2}$ & $\square_{2}$ & ${ }^{1} \mathbf{H}$ NMR & $\begin{array}{l}\text { \% Purity } \\
\text { LCMS } \\
214 \mathrm{~nm}\end{array}$ & Ionization & Calculated & Observed \\
\hline $61 \mathrm{a}$ & $\mathrm{A}$ & $\mathrm{H}$ & $\mathrm{A}$ & $\mathrm{H}$ & $\sqrt{ }$ & 86 & ES+ & $\left(\mathrm{M}+\mathrm{NH}_{4}^{+}\right) 447.2495$ & 447.2497 \\
\hline $61 b$ & A & $\mathrm{H}$ & B & $\mathrm{H}$ & $\sqrt{ }$ & 89 & ES+ & $\left(\mathrm{M}+\mathrm{Na}^{+}\right) 454.1842$ & 454.1857 \\
\hline $61 \mathrm{c}$ & A & $\mathrm{H}$ & $\mathrm{C}$ & $\mathrm{H}$ & $\sqrt{ }$ & $>90$ & ES+ & $\left(\mathrm{M}+\mathrm{Na}^{+}\right) 508.2675$ & 508.2670 \\
\hline 61d & A & $\mathrm{Br}$ & A & $\mathrm{H}$ & $\sqrt{ }$ & $>90$ & $\mathrm{ES}+$ & $\left(\mathrm{M}+\mathrm{Na}^{+}\right) 530.1154$ & 530.1169 \\
\hline $61 \mathrm{e}$ & $\mathrm{A}$ & $\mathrm{Br}$ & B & $\mathrm{H}$ & $\sqrt{ }$ & $>90$ & ES+ & $\left(\mathrm{M}+\mathrm{Na}^{+}\right) 532.0947$ & 532.0940 \\
\hline $61 f$ & A & $\mathrm{Br}$ & $\mathrm{C}$ & $\mathrm{H}$ & $\sqrt{ }$ & 90 & ES+ & $\left(\mathrm{M}+\mathrm{NH}_{4}^{+}\right) 581.2226$ & 581.2242 \\
\hline $61 \mathrm{~g}$ & A & $m-\mathrm{MePh}$ & $\mathrm{A}$ & $\mathrm{H}$ & $\sqrt{ }$ & $>90$ & ES+ & $\left(\mathrm{M}+\mathrm{NH}_{4}^{+}\right) 537.2964$ & 537.2972 \\
\hline $61 \mathrm{~h}$ & A & $m-\mathrm{MePh}$ & B & $\mathrm{H}$ & $\sqrt{ }$ & 89 & ES+ & $\left(\mathrm{M}+\mathrm{NH}_{4}^{+}\right) 539.2757$ & 539.2750 \\
\hline $61 i$ & A & $m-\mathrm{MePh}$ & $\mathrm{C}$ & $\mathrm{H}$ & $\sqrt{ }$ & 72 & ES+ & $\left(\mathrm{M}+\mathrm{NH}_{4}^{+}\right) 594.3591$ & 593.3590 \\
\hline $61 j$ & B & $\mathrm{H}$ & A & $\mathrm{H}$ & $\sqrt{ }$ & 81 & ES+ & $\left(\mathrm{M}+\mathrm{Na}^{+}\right) 573.2417$ & 574.2401 \\
\hline $61 \mathrm{k}$ & B & $\mathrm{H}$ & B & $\mathrm{H}$ & $\sqrt{ }$ & 81 & $\mathrm{ES}+$ & $\left(\mathrm{M}+\mathrm{Na}^{+}\right) 576.2210$ & 576.2219 \\
\hline 611 & B & $\mathrm{H}$ & $\mathrm{C}$ & $\mathrm{H}$ & $\sqrt{ }$ & 81 & ES+ & $\left(\mathrm{M}+\mathrm{Na}^{+}\right) 630.3043$ & 630.3035 \\
\hline $61 \mathrm{~m}$ & B & $\mathrm{Br}$ & A & $\mathrm{H}$ & $\sqrt{ }$ & 71 & ES+ & $\left(\mathrm{M}+\mathrm{Na}^{+}\right) 652.1522$ & 652.1506 \\
\hline $61 n$ & B & $\mathrm{Br}$ & B & $\mathrm{H}$ & $\sqrt{ }$ & 71 & ES+ & $\left(\mathrm{M}+\mathrm{Na}^{+}\right) 654.1315$ & 654.1289 \\
\hline 610 & B & $\mathrm{Br}$ & $\mathrm{C}$ & $\mathrm{H}$ & $\sqrt{ }$ & 79 & $\mathrm{ES}+$ & $\left(\mathrm{M}+\mathrm{Na}^{+}\right) 708.2148$ & 708.2156 \\
\hline $61 p$ & B & $m-\mathrm{MePh}$ & A & $\mathrm{H}$ & $\sqrt{ }$ & 76 & ES+ & $\left(\mathrm{M}+\mathrm{Na}^{+}\right) 664.2886$ & 664.2890 \\
\hline $61 q$ & B & $m-\mathrm{MePh}$ & B & $\mathrm{H}$ & $\sqrt{ }$ & 82 & ES+ & $\left(\mathrm{M}+\mathrm{Na}^{+}\right) 666.2757$ & 666.2742 \\
\hline $61 \mathrm{r}$ & B & $m-\mathrm{MePh}$ & $\mathrm{C}$ & $\mathrm{H}$ & $\sqrt{ }$ & $>90$ & ES+ & $\left(\mathrm{M}+\mathrm{Na}^{+}\right) 720.3512$ & 720.3529 \\
\hline $61 \mathrm{~s}$ & A & $\mathrm{H}$ & A & Ac & $\sqrt{ }$ & $>90$ & ES+ & $\left(\mathrm{M}+\mathrm{Na}^{+}\right) 494.2155$ & 494.2169 \\
\hline $61 t$ & A & $\mathrm{H}$ & B & Ac & $\sqrt{ }$ & 86 & ES+ & $\left(\mathrm{M}+\mathrm{Na}^{+}\right) 496.1947$ & 496.1951 \\
\hline $61 \mathrm{u}$ & A & $\mathrm{H}$ & $\mathrm{C}$ & Ac & $\sqrt{ }$ & $>90$ & ES+ & $\left(\mathrm{M}+\mathrm{Na}^{+}\right) 550.2781$ & 550.2798 \\
\hline $61 v$ & A & $\mathrm{Br}$ & A & Ac & $\sqrt{ }$ & $>90$ & ES+ & $\left(\mathrm{M}+\mathrm{Na}^{+}\right) 572.1260$ & 572.1277 \\
\hline $61 w$ & A & $\mathrm{Br}$ & B & Ac & $\sqrt{ }$ & $>90$ & ES+ & $\left(\mathrm{M}+\mathrm{Na}^{+}\right) 574.1052$ & 574.1057 \\
\hline $61 x$ & A & $\mathrm{Br}$ & $\mathrm{C}$ & Ac & $\sqrt{ }$ & $>90$ & $\mathrm{ES}+$ & $\left(\mathrm{M}+\mathrm{Na}^{+}\right) 628.1886$ & 628.1874 \\
\hline $61 y$ & A & $m-\mathrm{MePh}$ & A & Ac & $\sqrt{ }$ & $>90$ & $\mathrm{ES}+$ & $\left(\mathrm{M}+\mathrm{Na}^{+}\right) 584.2624$ & 584.2609 \\
\hline $61 z$ & A & $m-\mathrm{MePh}$ & B & Ac & $\sqrt{ }$ & $>90$ & $\mathrm{ES}+$ & $\left(\mathrm{M}+\mathrm{Na}^{+}\right) 586.2417$ & 586.2419 \\
\hline $61 \mathrm{aa}$ & A & $m-\mathrm{MePh}$ & $\mathrm{C}$ & Ac & $\sqrt{ }$ & 75 & ES+ & $\left(\mathrm{M}+\mathrm{Na}^{+}\right) 640.3250$ & 640.3244 \\
\hline $61 \mathrm{bb}$ & B & $\mathrm{H}$ & A & Ac & $\sqrt{ }$ & 77 & $\mathrm{ES}+$ & $\left(\mathrm{M}+\mathrm{Na}^{+}\right) 616.2523$ & 616.2524 \\
\hline 61cc & B & $\mathrm{H}$ & B & Ac & $\sqrt{ }$ & 81 & ES+ & $\left(\mathrm{M}+\mathrm{Na}^{+}\right) 618.2315$ & 618.2334 \\
\hline 61dd & B & $\mathrm{H}$ & $\mathrm{C}$ & Ac & $\sqrt{ }$ & 76 & ES+ & $\left(\mathrm{M}+\mathrm{Na}^{+}\right) 672.3149$ & 672.3134 \\
\hline 61ee & B & $\mathrm{Br}$ & A & Ac & $\sqrt{ }$ & 71 & ES+ & $\left(\mathrm{M}+\mathrm{Na}^{+}\right) 694.1628$ & 694.1645 \\
\hline $61 \mathrm{ff}$ & B & $\mathrm{Br}$ & B & Ac & $\sqrt{ }$ & 67 & ES+ & $\left(\mathrm{M}+\mathrm{Na}^{+}\right) 696.1420$ & 696.1391 \\
\hline $61 \operatorname{lgg}$ & B & $\mathrm{Br}$ & $\mathrm{C}$ & Ac & $\sqrt{ }$ & 73 & ES+ & $\left(\mathrm{M}+\mathrm{Na}^{+}\right) 750.2254$ & 750.2261 \\
\hline $61 \mathrm{hh}$ & B & $m-\mathrm{MePh}$ & A & Ac & $\sqrt{ }$ & 87 & ES+ & $\left(\mathrm{M}+\mathrm{Na}^{+}\right) 706.2992$ & 706.3015 \\
\hline 61ii & B & $m-\mathrm{MePh}$ & B & Ac & $\sqrt{ }$ & $>90$ & ES+ & $\left(\mathrm{M}+\mathrm{Na}^{+}\right) 708.2785$ & 708.2781 \\
\hline $61 \mathrm{jj}$ & B & $m-\mathrm{MePh}$ & $\mathrm{C}$ & Ac & $\sqrt{ }$ & $>90$ & ES+ & $\left(\mathrm{M}+\mathrm{Na}^{+}\right) 762.3618$ & 763.3609 \\
\hline
\end{tabular}

$\sqrt{ }: \quad{ }^{1} \mathrm{H}$ NMR spectrum consistent with anticipated structure 
Step 5. NBS and PPTS-mediated transformation of 61a-jj into a complete, combinatorial matrix of molecular skeletons, each derivatized with a complete, combinatorial matrix of building blocks.
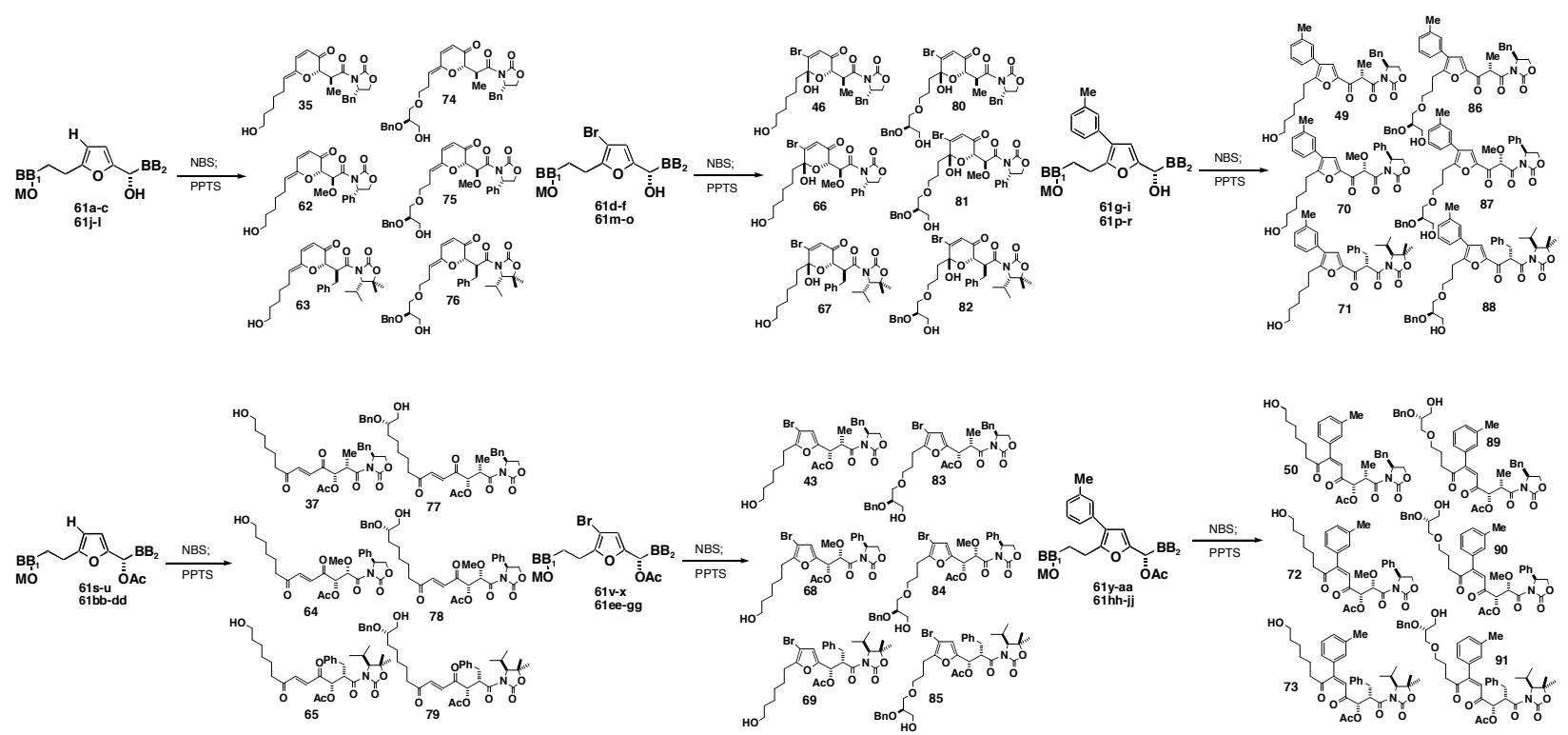

NBS and PPTS-mediated transformations. In 36 parallel reactions, each substrate $\mathbf{6 1 a}$-jj was subjected to the same reaction conditions (NBS/THF at $\mathrm{rt}$ for $1 \mathrm{~h}$; $\mathrm{PPTS} / \mathrm{CH}_{2} \mathrm{Cl}_{2}$ at $40-45{ }^{\circ} \mathrm{C}$ for $20 \mathrm{~h}$ ) using the same protocol described previously for the transformation of $\mathbf{3 2} \rightarrow \mathbf{3 5}$. The results are presented in tabular format below. 
Burke, Berger, and Schreiber - Supporting Information Part A

\section{Results of Step 5}

$\%$ Purity

Compound $\mathbf{B B}_{1} \quad \square_{1} \quad \mathbf{B B}_{2} \square_{2}$ 35

62

$63-\mathrm{A}-\mathrm{H}-\mathrm{C} \quad \mathrm{H}$

$37 \quad$ A $\quad$ H $\quad$ A $\quad$ Ac

$64 \quad \mathrm{~A} \quad \mathrm{H} \quad \mathrm{B} \quad \mathrm{Ac}$

65 A $\quad$ H $\quad$ C $\quad$ Ac

$\begin{array}{lllll}46 & \mathrm{~A} & \mathrm{Br} & \mathrm{A} & \mathrm{H}\end{array}$

$66 \quad \mathrm{~A} \quad \mathrm{Br} \quad \mathrm{B} \quad \mathrm{H}$

$\begin{array}{lllll}67 & \mathrm{~A} & \mathrm{Br} & \mathrm{C} & \mathrm{H}\end{array}$

$43 \quad \mathrm{~A} \quad \mathrm{Br} \quad \mathrm{A} \quad \mathrm{Ac}$

$68 \quad \mathrm{~A} \quad \mathrm{Br} \quad \mathrm{B} \quad \mathrm{Ac}$

$69 \quad \mathrm{~A} \quad \mathrm{Br} \quad \mathrm{C} \quad \mathrm{Ac}$

49 A $m-\mathrm{MePh}$ A $\mathrm{H}$

70 A $m-\mathrm{MePh}$ B $\mathrm{H}$

71 A $m-\mathrm{MePh} \quad \mathrm{C} \quad \mathrm{H}$

50 A $m$-MePh A $\mathrm{Ac}$

72 A $m-\mathrm{MePh}$ B $\mathrm{Ac}$

73 A $m-\mathrm{MePh} \mathrm{C}$ Ac

$\begin{array}{lllll}74 & \text { B } & \text { H } & \text { A } & \text { H }\end{array}$

$\begin{array}{lllll}75 & \text { B } & \text { H } & \text { B } & \text { H }\end{array}$

$\begin{array}{lllll}76 & \text { B } & \text { H } & \text { C } & \text { H }\end{array}$

$\begin{array}{lllll}77 & \text { B } & \text { H } & \text { A } & \text { Ac }\end{array}$

$\begin{array}{lllll}78 & \text { B } & \text { H } & \text { B } & \text { Ac }\end{array}$

$\begin{array}{lllll}79 & \mathrm{~B} & \mathrm{H} & \mathrm{C} & \mathrm{Ac}\end{array}$

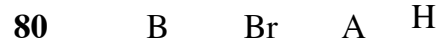

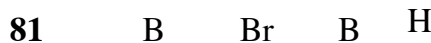

$82 \quad \mathrm{~B} \quad \mathrm{Br} \quad \mathrm{C} \quad \mathrm{H}$

$83 \quad$ B $\quad \mathrm{Br} \quad \mathrm{A} \quad \mathrm{Ac}$

$84 \quad \mathrm{~B} \quad \mathrm{Br} \quad \mathrm{B} \quad \mathrm{Ac}$

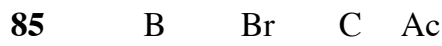

$86 \quad$ B $m-\mathrm{MePh} \quad \mathrm{A} \quad \mathrm{H}$

$87 \quad$ B $m-\mathrm{MePh} \quad$ B $\quad \mathrm{H}$

$88 \quad$ B $m-M e P h \quad C \quad H$

$89 \quad \mathrm{~B} \quad m-\mathrm{MePh} \quad \mathrm{A} \quad \mathrm{Ac}$

$90 \quad \mathrm{~B} \quad m-\mathrm{MePh} \quad \mathrm{B} \quad \mathrm{Ac}$

91

\section{${ }^{1} \mathrm{H}$ NMR}

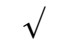

$\sqrt{ }$

LCMS, 214 nm Ionization

83

$>70$

$>80$

$>90$

85

78

$>90$ (10:1 e.r.)

$>90$ (1:1 e.r.)

89 (8:1 e.r)

$>90$

$>90$

$>90$

80

52

71

76

44

56

27

21

28

70

70

59

74 (8:1 e.r)

72 (3:1 e.r)

68 (8:1 e.r)

$$
76
$$

$>90$

$>90$

71

74

84

33

27

86

ES+

ES+

ES+

ES+

ES+

ES+

ES+

ES+

ES+

ES+

\section{HRMS}

\section{Calculated}

Observed

$\left(\mathrm{M}+\mathrm{H}^{+}\right) 428.2703$

428.2061

$\left(\mathrm{M}+\mathrm{Na}^{+}\right) 452.1685$

452.1700

484.2699

ES+ $\quad\left(\mathrm{M}+\mathrm{H}^{+}\right) 484.2699$

488.2275

$\left(\mathrm{M}+\mathrm{H}^{+}\right) 488.2284$

$\left(\mathrm{M}+\mathrm{NH}_{4}^{+}\right)$507. 2343

507.2358

$\left(\mathrm{M}+\mathrm{NH}_{4}^{+}\right) 561.3176$

561.3162

$\left(\mathrm{M}+\mathrm{Na}^{+}\right) 546.1103$

546.1086

$\left(\mathrm{M}+\mathrm{Na}^{+}\right) 548.0896$

548.0895

602.1733

$\left(\mathrm{M}+\mathrm{Na}^{+}\right) 602.1729$

572.1288

574.1038

$\left(\mathrm{M}+\mathrm{Na}^{+}\right) 574.1052$

623.2353

ES+ $\quad\left(\mathrm{M}+\mathrm{NH}_{4}{ }^{+}\right) 623.2332$

518.2532

$\left(\mathrm{M}+\mathrm{H}^{+}\right) 518.2542$

$\left(\mathrm{M}+\mathrm{Na}^{+}\right) 542.2155$

542.2152

$\left(\mathrm{M}+\mathrm{H}^{+}\right) 574.3168$

574.3163

$\left(\mathrm{M}+\mathrm{NH}_{4}^{+}\right) 595.3019$

595.3034

597.2803

$\left(\mathrm{M}+\mathrm{NH}_{4}{ }^{+}\right) 597.2812$

656.3177

ES+ $\quad\left(\mathrm{M}+\mathrm{Na}^{+}\right) 656.3199$

550.2437

$\mathrm{ES}+\quad\left(\mathrm{M}+\mathrm{H}^{+}\right) 550.2441$

552.2233

$\left(\mathrm{M}+\mathrm{H}^{+}\right) 552.2233$

606.3066

ES+ $\quad\left(\mathrm{M}+\mathrm{H}^{+}\right) 606.3067$

627.2930

612.2455

$\left(\mathrm{M}+\mathrm{H}^{+}\right) 612.2445$

666.3286

$\left(\mathrm{M}+\mathrm{H}^{+}\right) 666.3278$

663.1911

670.1268

$\left(\mathrm{M}+\mathrm{Na}^{+}\right) 670.1264$

719.2547

$\left(\mathrm{M}+\mathrm{NH}_{4}{ }^{+}+\right) 719.2543$

689.2081

$\left(\mathrm{M}+\mathrm{NH}_{4}^{+}\right) 689.2074$

691.1869

745.2704

$\left(\mathrm{M}+\mathrm{NH}_{4}^{+}\right) 745.2700$

640.2911

$\left(\mathrm{M}+\mathrm{H}^{+}\right) 640.2910$

642.2705

$\left(\mathrm{M}+\mathrm{H}^{+}\right) 642.2703$

696.3550

$\left(\mathrm{M}+\mathrm{H}^{+}\right) 696.3536$

717.3383

719.3179

$\left(\mathrm{M}+\mathrm{NH}_{4}{ }^{+}\right) 719.3180$

773.4014

$\sqrt{ }: \quad{ }^{1} \mathrm{H}$ NMR spectrum consistent with anticipated structure, e.r. $=$ epimeric ratio 


\section{Encoded split-pool synthesis}

\section{Step 1. Coupling and tagging of Building Block \#1 (BB 1$)$}

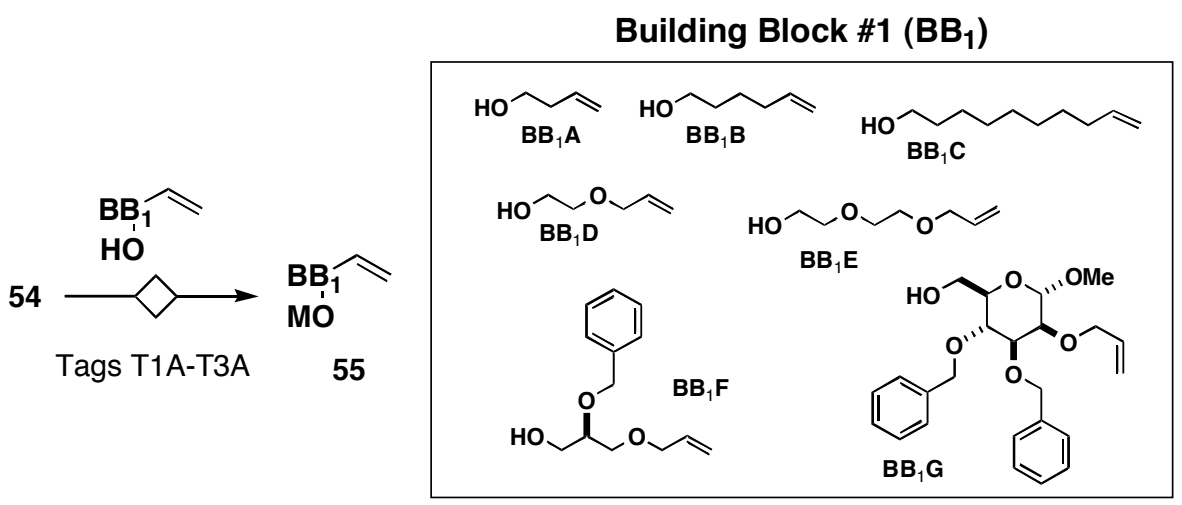

Coupling of $\mathbf{B B}_{1}$. A single pool of 3-[Diisopropyl(p-methoxyphenyl)silyl]-propyl functionalized beads 54 (2 g) was split evenly into seven portions ( $286 \mathrm{mg}$ each). Each portion of macrobeads 54 (286 $\mathrm{mg}$ per reaction) in a $10 \mathrm{~mL}$ polypropylene tube at $\mathrm{rt}$ under argon was allowed to swell in $\mathrm{CH}_{2} \mathrm{Cl}_{2}(7 \mathrm{ml})$ for $10 \mathrm{~min}$. The colorless beads were then filtered and again washed with $\mathrm{CH}_{2} \mathrm{Cl}_{2}$ (7 mL x $\left.10 \mathrm{~min}\right)$, and then resuspended in a $2.5 \%$ (v/v) solution of TMSCl in $\mathrm{CH}_{2} \mathrm{Cl}_{2}(7 \mathrm{~mL})$ for $30 \mathrm{~min}$. The beads were again filtered and washed thrice with $\mathrm{CH}_{2} \mathrm{Cl}_{2}(5$ min each) and then suspended in a $3 \%$ (v/v) solution of trifluoromethanesulfonic acid in $\mathrm{CH}_{2} \mathrm{Cl}_{2}$ $(6.6 \mathrm{~mL})$ for $20 \mathrm{~min}$ during which time the reaction tube was shaken periodically and the beads turned orange. After filtration, the orange-colored beads were again thrice washed with $\mathrm{CH}_{2} \mathrm{Cl}_{2}$ and then resuspended in a minimum volume of $\mathrm{CH}_{2} \mathrm{Cl}_{2}(\sim 1 \mathrm{~mL})$. Freshly distilled 2,6-lutidine was then added ( $346 \mathrm{uL}$, addition resulted in bead discoloration) followed by building block \# 1 (see below).

$B_{1}$ used in split-pool synthesis

$\begin{array}{cccccc}\text { Building Block } & \text { mol } & \text { formula weight }(\mathbf{g} / \mathbf{m o l}) & \text { density }(\mathbf{g} / \mathbf{m L}) & \text { volume }(\mathbf{u L}) & \text { mass }(\mathbf{g}) \\ \mathbf{B B}_{\mathbf{1}} \mathbf{A} & 0.002974 & 72.11 & 0.85 & 252 & \\ \mathbf{B B}_{\mathbf{1}} \mathbf{B} & 0.002974 & 100.16 & 0.834 & 357 & \\ \mathbf{B B}_{\mathbf{1}} \mathbf{C} & 0.002974 & 156.27 & 0.876 & 531 & \\ \mathbf{B B}_{\mathbf{1}} \mathbf{D} & 0.002974 & 102.13 & 0.955 & 318 \\ \mathbf{B B}_{\mathbf{1}} \mathbf{E} & 0.002237 & 146.68 & 1.01 & 325 & \\ \mathbf{B B}_{\mathbf{1}} \mathbf{F} & 0.002974 & 222.28 & \sim 1 & 661 & 0.661 \\ \mathbf{B B}_{\mathbf{1}} \mathbf{G} & 0.002974 & 414.49 & \mathrm{n} / \mathrm{a} & & 1.23^{*}\end{array}$

*dissolved in $300 \mathrm{uL} \mathrm{CH}_{2} \mathrm{Cl}_{2}$

The resulting colorless reaction mixtures were then shaken manually and let stand at $\mathrm{rt}$ for $16 \mathrm{~h}$. The beads were then filtered, washed with $\mathrm{CH}_{2} \mathrm{Cl}_{2}(5 \times 7 \mathrm{~mL} \times 20 \mathrm{~min}$. each), and the solvent was removed under argon flow followed by residual solvent removal in vacuo to yield seven portions of resin $\mathbf{5 5}$, each loaded with a unique $\mathbf{B B}_{\mathbf{1}}$. 
Tagging of $\mathbf{B B}_{1}$. The seven portions of resin $\mathbf{5 5}$ (loaded with $\mathbf{B B}_{1}$ ) were then each subjected to a unique encoding reaction. A freshly prepared solution of one or more tags (each tag $4.4 \mathrm{mM}$ in $\left.4.76 \mathrm{~mL} \mathrm{CH} \mathrm{CH}_{2}\right)$ was individually prepared for each reaction. The resin $\mathbf{5 5}(\sim 286 \mathrm{mg} / \mathrm{rxn})$ was then added to the solution of tags, placed under an argon cloud, capped and sealed with parafilm, and allowed to rotate gently at $\mathrm{rt}$ for $1 \mathrm{~h}$. To this mixture was then added a freshly prepared solution of rhodium triphenylacetate $(4.4 \mathrm{mg} / \mathrm{mL}, 4.76 \mathrm{~mL})$, and the vial was sealed under argon, wrapped in aluminum foil to prevent exposure to light, and allowed to tumble gently at rt for 15 h. The resin was then isolated by filtration and washed as follows: $2 \times\left(5 \times \mathrm{CH}_{2} \mathrm{Cl}_{2}, \mathrm{CH}_{2} \mathrm{Cl}_{2} \times 15\right.$ min.), $3 \times(5 \times \mathrm{THF}$, THF $\times 2 \mathrm{~h}), 5 \times$ anhydrous $\mathrm{CH}_{2} \mathrm{Cl}_{2}$, anhydrous $\mathrm{CH}_{2} \mathrm{Cl}_{2} \times 15 \mathrm{~min}$. The solvent was then removed under argon flow for $1 \mathrm{~h}$ followed by residual solvent removal in vacuo to yield seven portions of resin $\mathbf{5 5}$ loaded with $\mathbf{B B}_{1}$ and chemically encoded with polychlorinated aromatic tags T1A-T3A.

Encoding scheme for $\mathrm{BB}_{1}$

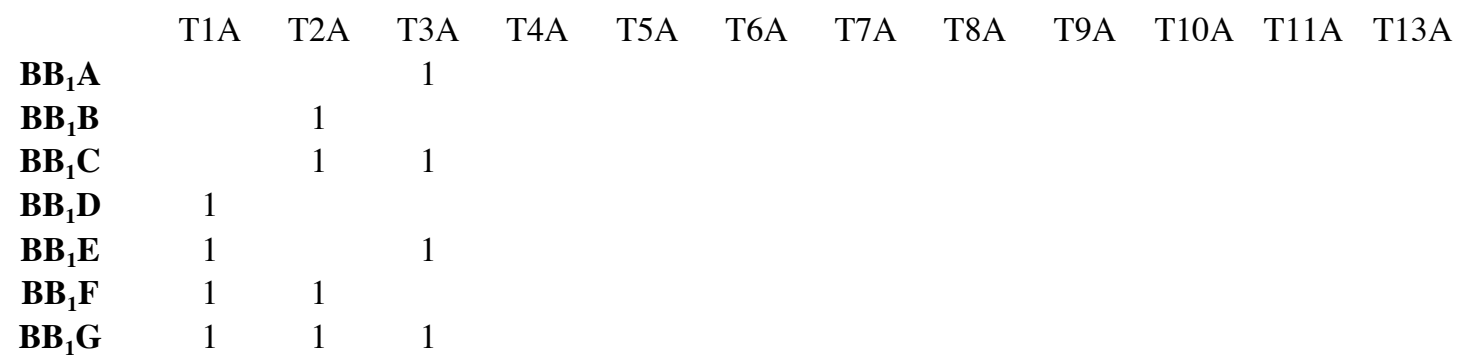

Two macrobeads from each of the seven portions were removed and subjected to the standard HF-Pyridine-mediated compound cleavage conditions (see General Information), and the individual macrobeads and/or a portion of the solution of cleaved compounds were subsequently subjected to the standard CAN-mediated tag cleavage reaction. ${ }^{1}$ After confirming tagging scheme, the seven portions of dry resin $\mathbf{5 5}$ were pooled together in a single polypropylene tube, swollen in anh. THF, tumbled for $30 \mathrm{~min}$, and then the solvent was removed under argon flow followed by residual solvent removal in vacuo.

${ }^{1}$ H.E. Blackwell and coworkers, Chem. Biol. 2001, 8, 1167. 
Step 2. Coupling and tagging of Skeletal Information Element \#1 $\left(\square_{1}\right)$

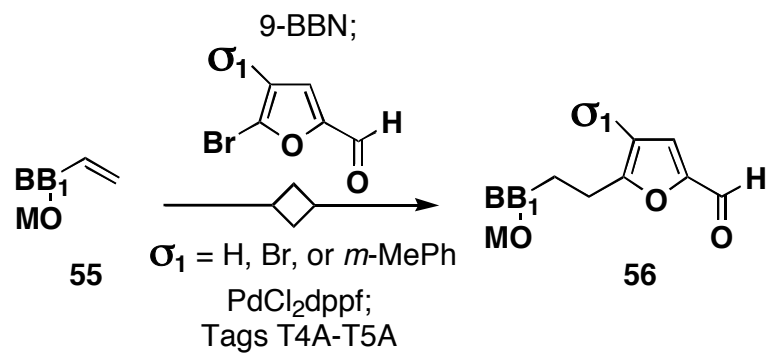

Coupling of $\square_{1}$. A single pool of resin 55 was split evenly into three portions (672 $\mathrm{mg}$ each). Each portion of colorless beads $55(672 \mathrm{mg})$ were washed with THF ( 2 x $15 \mathrm{~mL}$ x $10 \mathrm{~min}$ each) at $\mathrm{rt}$ and then resuspended in $20.2 \mathrm{~mL}$ THF. A $0.5 \mathrm{M}$ solution of $9-\mathrm{BBN}$ in THF $(13.4 \mathrm{~mL}, 6.72$ mmol) was then added and the resulting mixture was manually agitated and let stand at $\mathrm{rt}$ for $5 \mathrm{~h}$. The reaction solution was then removed via cannula and the colorless resin was washed thoroughly with THF (5 x $15 \mathrm{~mL}$ x $10 \mathrm{~min}$ each). To the resin was then added solid $\mathrm{PdCl}_{2} \mathrm{dppf}$ $\left(\square_{1}=\mathrm{H}: 8.2 \mathrm{mg}, 0.0101 \mathrm{mmol} ; \square_{1}=\mathrm{Br}: 13.7 \mathrm{mg}, 0.0168 \mathrm{mmol} ; \square_{1}=\right.$ Ar: $\left.8.2 \mathrm{mg}, 0.0101 \mathrm{mmol}\right)$, and one of the following three 5-bromofuraldehydes:

$\square_{1}$ used in split-pool synthesis

$\begin{array}{cccc}\text { Skeletal information element }(\square) & \text { mmol } & \text { formula weight }(\mathbf{g} / \mathbf{m o l}) & \text { mass }(\mathbf{g}) \\ \square_{\mathbf{1}} \mathbf{A}, \text { 5-Bromofuraldehyde } & 3.36 & 174.99 & 0.5880 \\ \square_{\mathbf{1}} \mathbf{B}, 4,5 \text {-Dibromofuraldehyde } & 3.36 & 253.88 & 0.8530 \\ \square_{\mathbf{1}} \mathbf{C}, 4-m-\mathrm{MePh}-5 \text {-bromofuraldehyde } & 3.36 & 265.1 & 0.8907\end{array}$

via cannula as a solution in THF $(8.4 \mathrm{~mL})$, and a $1 \mathrm{M}$ solution of $\mathrm{NaOH}(1.68 \mathrm{~mL}, 1.68 \mathrm{mmol})$. The resulting orange reaction mixture was sealed under a cloud of argon and heated at $65{ }^{\circ} \mathrm{C}$ with periodic manual agitation for $20 \mathrm{~h}$ (each reaction mixture turned dark brown over several hours). The yellow/orange resin was then isolated by filtration and washed as follows, $5 \mathrm{x}$ THF, $5 \mathrm{x}$ $\mathrm{H}_{2} \mathrm{O}, 5 \times$ THF, THF: $\mathrm{H}_{2} \mathrm{O} 3: 1 \times 2$ h, 5 x THF, 3 x $\mathrm{H}_{2} \mathrm{O}, 5$ x THF, THF: $\mathrm{H}_{2} \mathrm{O} 3: 1$ x 45min, 5 x THF, THF: $\mathrm{H}_{2} \mathrm{O} 3: 1$ x $45 \mathrm{~min}, 5$ x THF, THF x $20 \mathrm{~min}, 5$ x $\mathrm{CH}_{2} \mathrm{Cl}_{2}, \mathrm{CH}_{2} \mathrm{Cl}_{2}$ x $20 \mathrm{~min}, 5$ x anh. $\mathrm{CH}_{2} \mathrm{Cl}_{2}$, anh. $\mathrm{CH}_{2} \mathrm{Cl}_{2} \times 20 \mathrm{~min}$, and then the solvent was removed under argon flow followed by residual solvent removal in vacuo to yield three portions of yellow/orange product resin $\mathbf{5 6}$.

Tagging of $\square_{1}$. Each of the three product portions 56 was then subjected to a unique encoding reaction. A freshly prepared solution of one or more tags (each tag $4.4 \mathrm{mM}$ in $11.1 \mathrm{~mL} \mathrm{CH}_{2} \mathrm{Cl}_{2}$ ) was individually prepared for each reaction. The resin 56 (>672 mg/rxn) was then added to the solution of tags, placed under an argon cloud, capped and sealed with parafilm, and allowed to rotate gently for $1 \mathrm{~h}$. To this mixture was then added a freshly prepared solution of rhodium triphenylacetate $(4.4 \mathrm{mg} . / \mathrm{mL}, 11.1 \mathrm{~mL})$, and the vial was sealed under argon, wrapped in aluminum foil to prevent exposure to light, and allowed to tumble gently for $15 \mathrm{~h}$. The resin was then isolated by filtration and washed as follows: $2 \times\left(5 \times \mathrm{CH}_{2} \mathrm{Cl}_{2}, \mathrm{CH}_{2} \mathrm{Cl}_{2} \times 15\right.$ min. $), 3 \times(5 \times$ THF, THF x 2 h), 5 x anh. THF, anh. THF x 1 h, 5 x anh. $\mathrm{CH}_{2} \mathrm{Cl}_{2}$, anh. $\mathrm{CH}_{2} \mathrm{Cl}_{2} \times 20 \mathrm{~min}$, and the solvent was removed under argon flow followed by residual solvent removal in vacuo to yield 
three portions of resin $\mathbf{5 6}$, collectively representing all combinations of $\mathbf{B B}_{\mathbf{1}}$ and $\square_{1}$, with each combination chemically encoded with polychlorinated aromatic tags.

Encoding scheme for $\square_{1}$

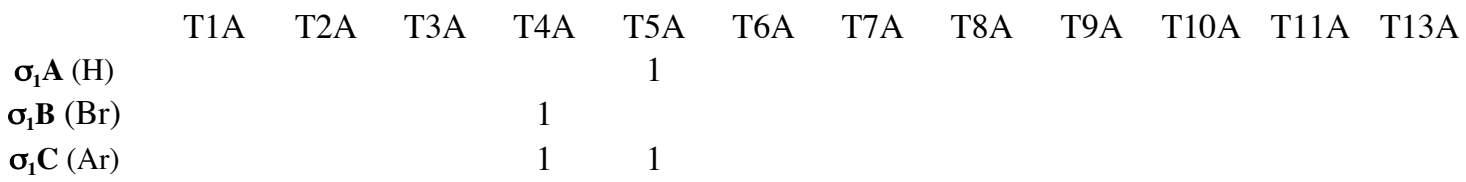

Ten individual macrobeads were removed from each portion $\mathbf{5 6}$ and subjected to the standard HF-Pyridine cleavage conditions. The cleaved product from all 30 individual macrobeads was analyzed by LCMS, and the polychlorinated tags remaining on each macrobead were then cleaved and analyzed by GC (tagging scheme was confirmed, data not shown). The three pools of dry resin $\mathbf{5 6}$ were then pooled together in a single polypropylene tube, swollen in anh. $\mathrm{CH}_{2} \mathrm{Cl}_{2}$, tumbled for $30 \mathrm{~min}$, and then the solvent was removed under argon flow followed by residual solvent removal in vacuo. 
Step 3. Coupling and tagging of Building Block \#2 (BB $\left.{ }_{2}\right)$

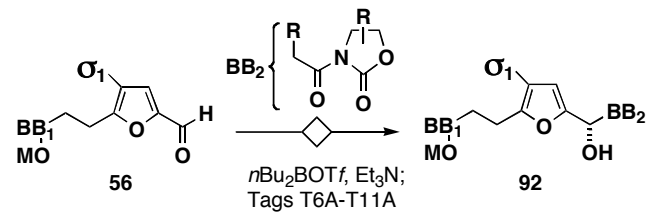

Building Block \#2 $\left(\mathrm{BB}_{2}\right)$

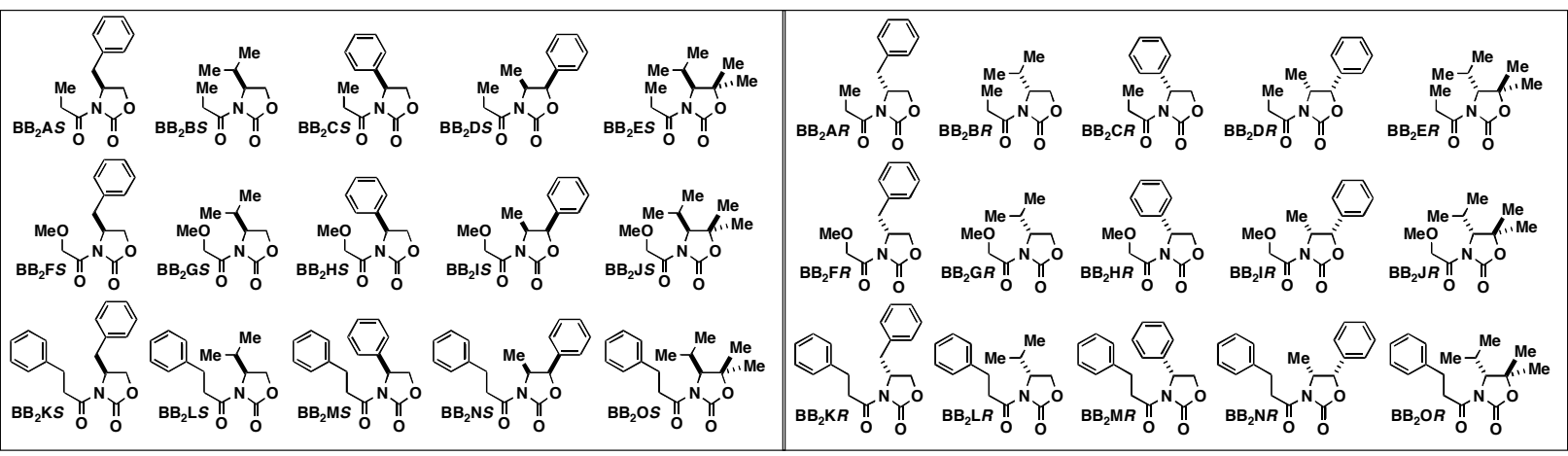

Coupling of $\mathbf{B B}_{2}$. The pooled resin $\mathbf{5 6}$ from Step 2 was then split into 30 equal portions (73.5 $\mathrm{mg}$ each). Each portion of resin $\mathbf{5 6}(73.5 \mathrm{mg})$ was washed with $\mathrm{CH}_{2} \mathrm{Cl}_{2}(2 \times 3 \mathrm{~mL} \times 10 \mathrm{~min}$ each) at $\mathrm{rt}$, and then cooled to $-78{ }^{\circ} \mathrm{C}$. In a separate vessel, to a stirred solution of acyl oxazolidinone ( $0.75 \mathrm{mmol}$, each was azeotropically dried from benzene just prior to reaction, see table below):

$\mathrm{BB}_{2}$ used in split-pool synthesis

\begin{tabular}{|c|c|c|c|c|c|c|c|}
\hline $\mathbf{B B}_{2}$ & mmol & FW (g/mol) & mass (g) & $\mathbf{B B}_{2}$ & mmol & FW (g/mol) & mass (g) \\
\hline $\mathrm{BB}_{2} \mathrm{~A} S$ & 0.75 & 233.26 & 0.175 & $\mathrm{BB}_{2} \mathrm{~A} R$ & 0.75 & 233.27 & 0.175 \\
\hline $\mathrm{BB}_{2} \mathrm{~B} S$ & 0.75 & 185.22 & 0.1389 & $\mathrm{BB}_{2} \mathrm{~B} R$ & 0.75 & 185.22 & 0.1389 \\
\hline $\mathrm{BB}_{2} \mathrm{CS}$ & 0.75 & 219.24 & 0.1644 & $\mathrm{BB}_{2} \mathrm{CR}$ & 0.75 & 219.24 & 0.1644 \\
\hline $\mathrm{BB}_{2} \mathrm{DS}$ & 0.75 & 233.26 & 0.175 & $\mathrm{BB}_{2} \mathrm{DR}$ & 0.75 & 233.26 & 0.175 \\
\hline $\mathrm{BB}_{2} \mathrm{E} S$ & 0.75 & 213.27 & 0.16 & $\mathrm{BB}_{2} \mathrm{E} R$ & 0.75 & 213.27 & 0.16 \\
\hline $\mathrm{BB}_{2} \mathrm{FS}$ & 0.75 & 249.26 & 0.1869 & $\mathrm{BB}_{2} \mathrm{FR}$ & 0.75 & 249.26 & 0.1869 \\
\hline $\mathrm{BB}_{2} \mathbf{G} S$ & 0.75 & 201.22 & 0.1509 & $\mathbf{B B}_{2} \mathbf{G} \boldsymbol{R}$ & 0.75 & 201.22 & 0.1509 \\
\hline $\mathrm{BB}_{2} \mathrm{HS}$ & 0.75 & 235.24 & 0.1764 & $\mathrm{BB}_{2} \mathrm{H} R$ & 0.75 & 235.24 & 0.1764 \\
\hline $\mathbf{B B}_{2} \mathbf{I} S$ & 0.75 & 249.26 & 0.1869 & $\mathbf{B B}_{2} \mathbf{I} R$ & 0.75 & 249.26 & 0.1869 \\
\hline $\mathrm{BB}_{2} \mathrm{~J} S$ & 0.75 & 229.27 & 0.172 & $\mathbf{B B}_{2} \mathrm{~J} R$ & 0.75 & 229.27 & 0.172 \\
\hline $\mathrm{BB}_{2} \mathrm{~K} S$ & 0.75 & 309.36 & 0.232 & $\mathrm{BB}_{2} \mathrm{~K} R$ & 0.75 & 309.36 & 0.232 \\
\hline $\mathrm{BB}_{2} \mathrm{LS}$ & 0.75 & 261.32 & 0.196 & $\mathrm{BB}_{2} \mathrm{LR}$ & 0.75 & 261.32 & 0.196 \\
\hline $\mathrm{BB}_{2} \mathbf{M} S$ & 0.75 & 295.33 & 0.2215 & $\mathrm{BB}_{2} \mathrm{MR}$ & 0.75 & 295.33 & 0.2215 \\
\hline $\mathrm{BB}_{2} \mathrm{~N} S$ & 0.75 & 309.36 & 0.232 & $\mathrm{BB}_{2} \mathrm{~N} R$ & 0.75 & 309.36 & 0.232 \\
\hline $\mathrm{BB}_{2} \mathrm{OS}$ & 0.75 & 289.37 & 0.217 & $\mathrm{BB}_{2} \mathrm{OR}$ & 0.75 & 289.37 & 0.217 \\
\hline
\end{tabular}

in $\mathrm{CH}_{2} \mathrm{Cl}_{2}(3 \mathrm{~mL})$ at $0{ }^{\circ} \mathrm{C}$ was added a $1 \mathrm{M}$ solution of dibutylboron triflate in $\mathrm{CH}_{2} \mathrm{Cl}_{2}(0.788 \mathrm{~mL}$, $0.788 \mathrm{mmol})$ followed by triethylamine $(0.125 \mathrm{~mL}, 0.900 \mathrm{mmol})$. The resulting enolate solution was cooled to $-78{ }^{\circ} \mathrm{C}$ and then transferred rapidly via cannula to the vessel containing 56. The resulting mixture was sealed under a cloud of argon and maintained at $-78{ }^{\circ} \mathrm{C}$ for $48 \mathrm{~h}(72 \mathrm{~h}$ for 
$\mathrm{BB}_{2} \mathrm{MS}, \mathrm{BB}_{2} \mathrm{OS}, \mathbf{B B}_{2} \mathbf{M} \boldsymbol{R}, \mathbf{B B}_{2} \mathbf{N R}$, and $\mathbf{B B}_{2} \mathrm{OR}$ ) $-26{ }^{\circ} \mathrm{C}$ for $24 \mathrm{~h}$, and $0{ }^{\circ} \mathrm{C}$ for $2 \mathrm{~h}$ (with periodic manual agitation about once every $8 \mathrm{~h}$ ). The reaction was then quenched with the addition of pH7 phosphate buffer $(3 \mathrm{~mL}), \mathrm{MeOH}(3 \mathrm{~mL})$, and $30 \%$ aq. $\mathrm{H}_{2} \mathrm{O}_{2}(2 \mathrm{~mL})$, and the resulting mixture was tumbled at $4{ }^{\circ} \mathrm{C}$ for $12-15 \mathrm{~h}$. Resin was then isolated by filtration and washed as follows: $5 \times \mathrm{CH}_{2} \mathrm{Cl}_{2}, 5 \times \mathrm{DMF}, 5 \times \mathrm{THF}, 5 \times \mathrm{CH}_{2} \mathrm{Cl}_{2}, \mathrm{CH}_{2} \mathrm{Cl}_{2} \times 1 \mathrm{~h}, 5 \times \mathrm{DMF}$, DMF x $1 \mathrm{~h}, 5 \mathrm{x}$ THF, THF x $1 \mathrm{~h}, 5 \times \mathrm{CH}_{2} \mathrm{Cl}_{2}, \mathrm{CH}_{2} \mathrm{Cl}_{2} \times 30 \mathrm{~min}, 5 \times$ anhydrous $\mathrm{CH}_{2} \mathrm{Cl}_{2}$, anhydrous $\mathrm{CH}_{2} \mathrm{Cl}_{2} \times 30$ min, and the solvent was removed under argon flow followed by residual solvent removal in vacuo to yield yellow product resin 92.

Tagging of $\mathbf{B B}_{2}$. Each of the 30 portions of product resin 92 loaded with $\square \square_{2}$ was then subjected to a unique encoding reaction. A freshly prepared solution of one or more tags (each tag $4.4 \mathrm{mM}$ in $\left.1.1 \mathrm{~mL} \mathrm{CH}_{2} \mathrm{Cl}_{2}\right)$ was individually prepared for each reaction. The resin $92(>73.5 \mathrm{mg} / \mathrm{rxn})$ was then added to the solution of tags, placed under an argon cloud, capped and sealed with parafilm, and allowed to rotate gently for $1 \mathrm{~h}$. To this mixture was then added a freshly prepared solution of rhodium triphenylacetate $(4.4 \mathrm{mg} . / \mathrm{mL}, 1.1 \mathrm{~mL})$, and the vial was sealed under argon, wrapped in aluminum foil to prevent exposure to light, and allowed to tumble gently for $15 \mathrm{~h}$. The resin was then isolated by filtration and washed as follows: $2 \times\left(5 \times \mathrm{CH}_{2} \mathrm{Cl}_{2}, \mathrm{CH}_{2} \mathrm{Cl}_{2} \times 15 \mathrm{~min}\right.$.), 3 x (5 x THF, THF x 2 h), 5 x anh. THF, anh. THF x 1 h, 5 x anh. $\mathrm{CH}_{2} \mathrm{Cl}_{2}$, anh. $\mathrm{CH}_{2} \mathrm{Cl}_{2}$ x 20 min, and the solvent was removed under argon flow followed by residual solvent removal in vacuo to yield 30 portions of resin 92 representing all combinations of $\mathbf{B B}_{1}, \mathbf{\square}_{1}$, and $\mathbf{B B}_{2}$, with each combination chemically encoded with polychlorinated aromatic tags. 
Encoding scheme for $\mathrm{BB}_{2}$

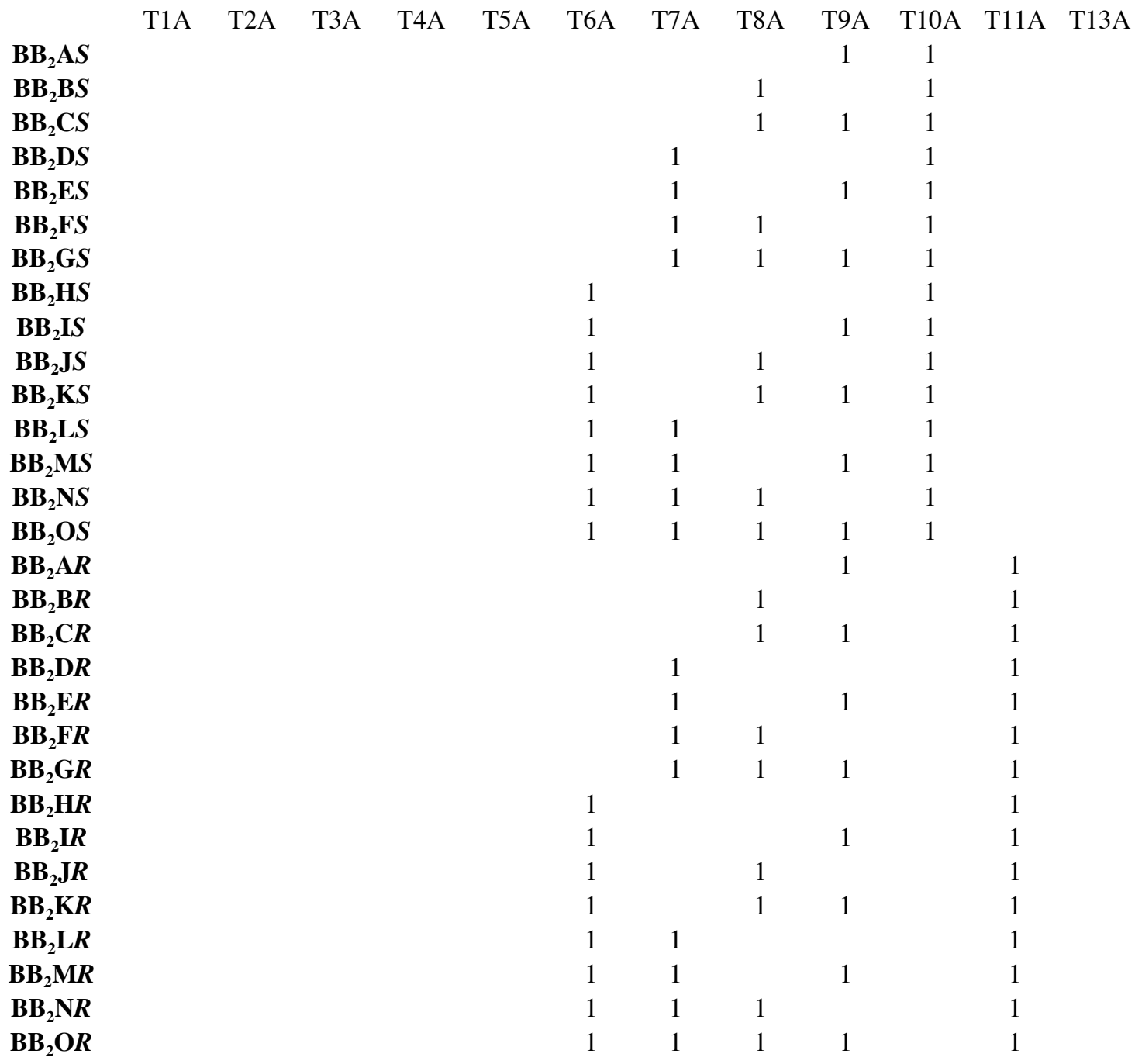

Two individual macrobeads were removed from each portion of product resin 92 and subjected to the standard HF-Pyridine cleavage conditions. The cleaved product from each of these 60 individual macrobeads was analyzed by LCMS, and the polychlorinated tags remaining on each macrobead were then cleaved and analyzed by GC. The results are presented in tabular format below. Having confirmed the tagging scheme, the 30 portions of dry resin 92 were then pooled together in a single polypropylene tube and well-mixed. 
Burke, Berger, and Schreiber - Supporting Information Part A

\section{Results of Step 3}

\begin{tabular}{|c|c|c|c|c|c|c|c|c|}
\hline \multirow[b]{2}{*}{ Macrobead } & \multicolumn{3}{|c|}{$\begin{array}{l}\text { Structure encoded by } \\
\text { chemical tags }\end{array}$} & \multirow{2}{*}{$\begin{array}{l}\text { \% Purity, } \\
\text { LCMS } \\
\text { (214 nm) }\end{array}$} & \multicolumn{3}{|c|}{ ES+ Mass spec } & \multirow{2}{*}{$\begin{array}{c}\text { consistent } \\
\text { with tag- } \\
\text { encoded } \\
\text { structure }\end{array}$} \\
\hline & $\mathbf{B B}_{1}$ & $\square_{1}$ & $\mathbf{B B}_{2}$ & & Ion & Calculated & Observed & \\
\hline $92 a$ & $\mathrm{~A}$ & $\mathrm{C}$ & $\mathrm{A} S$ & $>90$ & $\mathrm{M}+\mathrm{Na}^{+}$ & 514 & 514 & $\sqrt{ }$ \\
\hline $92 b$ & $\mathrm{E}$ & $\mathrm{B}$ & $\mathrm{A} S$ & $>90$ & $\mathrm{M}+\mathrm{Na}^{+}$ & 576 & 576 & $\sqrt{ }$ \\
\hline $92 \mathrm{c}$ & $\mathrm{A}$ & $\mathrm{C}$ & $\mathrm{B} S$ & $>90$ & $\mathrm{M}+\mathrm{Na}^{+}$ & 466 & 466 & $\sqrt{ }$ \\
\hline 92d & $\mathrm{E}$ & B & $\mathrm{B} S$ & $>90$ & $\mathrm{M}+\mathrm{Na}^{+}$ & 528 & 528 & $\sqrt{ }$ \\
\hline $92 e$ & B & $\mathrm{A}$ & $\mathrm{C} S$ & $>90$ & $\mathrm{M}+\mathrm{Na}^{+}$ & 438 & 438 & $\sqrt{ }$ \\
\hline $92 f$ & B & $\mathrm{C}$ & $\mathrm{C} S$ & 90 & $\mathrm{M}+\mathrm{Na}^{+}$ & 528 & 528 & $\sqrt{ }$ \\
\hline $92 \mathrm{~g}$ & $\mathrm{D}$ & $\mathrm{A}$ & $\mathrm{D} S$ & $>90$ & $\mathrm{M}+\mathrm{Na}^{+}$ & 454 & 454 & $\sqrt{ }$ \\
\hline $92 \mathrm{~h}$ & A & $\mathrm{C}$ & $\mathrm{D} S$ & $>90$ & $\mathrm{M}+\mathrm{Na}^{+}$ & 514 & 514 & $\sqrt{ }$ \\
\hline $92 \mathrm{i}$ & $\mathrm{G}$ & $\mathrm{A}$ & $\mathrm{E} S$ & 67 & $\mathrm{M}+\mathrm{Na}^{+}$ & 746 & 746 & $\sqrt{ }$ \\
\hline $92 j$ & $\mathrm{~A}$ & $\mathrm{~B}$ & $\mathrm{E} S$ & $>90$ & $\mathrm{M}+\mathrm{Na}^{+}$ & 482 & 482 & $\sqrt{ }$ \\
\hline $92 k$ & $\mathrm{C}$ & $\mathrm{C}$ & FS & 88 & $\mathrm{M}+\mathrm{Na}^{+}$ & 614 & 614 & $\sqrt{ }$ \\
\hline 921 & $\mathrm{G}$ & B & FS & 55 & $\mathrm{M}+\mathrm{NH}_{4}^{+}$ & 855 & 855 & $\sqrt{ }$ \\
\hline $92 m$ & $\mathrm{G}$ & A & $\mathrm{G} S$ & 56 & $\mathrm{M}+\mathrm{Na}^{+}$ & 734 & 734 & $\sqrt{ }$ \\
\hline $92 n$ & $\mathrm{~A}$ & A & GS & $>90$ & $\mathrm{M}+\mathrm{Na}^{+}$ & 392 & 392 & $\sqrt{ }$ \\
\hline 920 & $\mathrm{C}$ & A & $\mathrm{H} S$ & $>90$ & $\mathrm{M}+\mathrm{Na}^{+}$ & 510 & 510 & $\sqrt{ }$ \\
\hline $92 p$ & B & $\mathrm{C}$ & $\mathrm{H} S$ & 88 & $\mathrm{M}+\mathrm{Na}^{+}$ & 544 & 544 & $\sqrt{ }$ \\
\hline $92 q$ & $\mathrm{D}$ & $\mathrm{C}$ & IS & 87 & $\mathrm{M}+\mathrm{Na}^{+}$ & 560 & 560 & $\sqrt{ }$ \\
\hline $92 \mathrm{r}$ & $\mathrm{F}$ & $\mathrm{C}$ & IS & 88 & $\mathrm{M}+\mathrm{Na}^{+}$ & 680 & 680 & $\sqrt{ }$ \\
\hline $92 \mathrm{~s}$ & $\mathrm{C}$ & B & $\mathrm{J} S$ & $>90$ & $\mathrm{M}+\mathrm{Na}^{+}$ & 582 & 582 & $\sqrt{ }$ \\
\hline $92 t$ & $\mathrm{D}$ & $\mathrm{C}$ & $\mathrm{J} S$ & $>90$ & $\mathrm{M}+\mathrm{Na}^{+}$ & 540 & 540 & $\sqrt{ }$ \\
\hline $92 u$ & E & $\mathrm{C}$ & $\mathrm{K} S$ & $>90$ & $\mathrm{M}+\mathrm{Na}^{+}$ & 664 & 664 & $\sqrt{ }$ \\
\hline $92 v$ & $\mathrm{C}$ & A & $\mathrm{K} S$ & $>90$ & $\mathrm{M}+\mathrm{Na}^{+}$ & 584 & 584 & $\sqrt{ }$ \\
\hline $92 w$ & E & $\mathrm{C}$ & $\mathrm{L} S$ & 85 & $\mathrm{M}+\mathrm{Na}^{+}$ & 616 & 616 & $\sqrt{ }$ \\
\hline $92 x$ & $\mathrm{C}$ & A & $\mathrm{L} S$ & 90 & $\mathrm{M}+\mathrm{Na}^{+}$ & 536 & 536 & $\sqrt{ }$ \\
\hline $92 y$ & $\mathrm{G}$ & B & $\mathrm{M} S$ & 58 & $\mathrm{M}+\mathrm{NH}_{4}^{+}$ & 901 & 901 & $\sqrt{ }$ \\
\hline $92 z$ & $\mathrm{G}$ & A & $\mathrm{M} S$ & 74 & $\mathrm{M}+\mathrm{NH}_{4}^{+}$ & 823 & 823 & $\sqrt{ }$ \\
\hline 92aa & $\mathrm{G}$ & $\mathrm{C}$ & $\mathrm{N} S$ & ND & $\mathrm{M}+\mathrm{NH}_{4}^{+}$ & 927 & 927 & $\sqrt{ }$ \\
\hline 92bb & $\mathrm{E}$ & $\mathrm{C}$ & $\mathrm{N} S$ & 82 & $\mathrm{M}+\mathrm{Na}^{+}$ & 664 & 664 & $\sqrt{ }$ \\
\hline $92 \mathrm{cc}$ & $\mathrm{E}$ & $\mathrm{A}$ & OS & 91 & $\mathrm{M}+\mathrm{Na}^{+}$ & 554 & 554 & $\sqrt{ }$ \\
\hline 92dd & $\mathrm{F}$ & A & $\mathrm{OS}$ & 66 & $\mathrm{M}+\mathrm{Na}^{+}$ & 630 & 630 & $\sqrt{ }$ \\
\hline 92ee & B & $\mathrm{C}$ & $\mathrm{A} R$ & 86 & $\mathrm{M}+\mathrm{Na}^{+}$ & 542 & 542 & $\sqrt{ }$ \\
\hline $92 \mathrm{ff}$ & E & B & $\mathrm{A} R$ & $>90$ & $\mathrm{M}+\mathrm{Na}^{+}$ & 576 & 576 & $\sqrt{ }$ \\
\hline 92gg & F & $\mathrm{C}$ & $\mathrm{B} R$ & 83 & $\mathrm{M}+\mathrm{Na}^{+}$ & 616 & 616 & $\sqrt{ }$ \\
\hline $92 \mathrm{hh}$ & $\mathrm{G}$ & A & $\mathrm{B} R$ & 78 & $\mathrm{M}+\mathrm{Na}^{+}$ & 718 & 718 & $\sqrt{ }$ \\
\hline 92ii & $\mathrm{E}$ & A & $\mathrm{CR}$ & $>90$ & $\mathrm{M}+\mathrm{Na}^{+}$ & 484 & 484 & $\sqrt{ }$ \\
\hline 92jj & $\mathrm{D}$ & $\mathrm{C}$ & $\mathrm{C} R$ & 84 & $\mathrm{M}+\mathrm{Na}^{+}$ & 530 & 430 & $\sqrt{ }$ \\
\hline
\end{tabular}


Burke, Berger, and Schreiber - Supporting Information Part A

\begin{tabular}{|c|c|c|c|c|c|c|c|c|}
\hline Macrobead & $\mathbf{B B}_{1}$ & $\square_{1}$ & $\mathbf{B B}_{2}$ & $\begin{array}{c}(\square=214 \\
\text { nm })\end{array}$ & Ion & Calculated & Observed & $\begin{array}{c}\text { encoded } \\
\text { structure }\end{array}$ \\
\hline 92kk & $\mathrm{G}$ & A & $\mathrm{D} R$ & 86 & $\mathrm{M}+\mathrm{NH}_{4}^{+}$ & 761 & 761 & $\sqrt{ }$ \\
\hline 9211 & G & A & $\mathrm{D} R$ & 81 & $\mathrm{M}+\mathrm{NH}_{4}^{+}$ & 761 & 761 & $\sqrt{ }$ \\
\hline $92 \mathrm{~mm}$ & D & $C$ & $\mathrm{E} R$ & 71 & $\mathrm{M}+\mathrm{Na}^{+}$ & 524 & 524 & $\sqrt{ }$ \\
\hline $92 \mathrm{nn}$ & A & B & $\mathrm{E} R$ & 75 & $\mathrm{M}+\mathrm{Na}^{+}$ & 482 & 482 & $\sqrt{ }$ \\
\hline 9200 & E & $\mathrm{C}$ & $\mathrm{F} R$ & 89 & $\mathrm{M}+\mathrm{Na}^{+}$ & 604 & 604 & $\sqrt{ }$ \\
\hline 92pp & $\mathrm{C}$ & $A$ & $\mathrm{~F} R$ & $>90$ & $\mathrm{M}+\mathrm{Na}^{+}$ & 524 & 524 & $\sqrt{ }$ \\
\hline $92 q q$ & ND & ND & ND & 89 & ND & ND & ND & ND \\
\hline 92rr & $\mathrm{C}$ & A & $\mathrm{G} R$ & $>90$ & $\mathrm{M}+\mathrm{Na}^{+}$ & 476 & 476 & $\sqrt{ }$ \\
\hline 92ss & $\mathrm{F}$ & B & $\mathrm{H} R$ & $>90$ & $\mathrm{M}+\mathrm{Na}^{+}$ & 654 & 654 & $\sqrt{ }$ \\
\hline $92 \mathrm{tt}$ & ND & ND & ND & $>90$ & ND & ND & ND & ND \\
\hline 92uu & $\mathrm{A}$ & B & $\mathrm{I} R$ & $>90$ & $\mathrm{M}+\mathrm{Na}^{+}$ & 518 & 518 & $\sqrt{ }$ \\
\hline $92 \mathrm{vv}$ & $\mathrm{F}$ & B & $\mathrm{I} R$ & $>90$ & $\mathrm{M}+\mathrm{Na}^{+}$ & 668 & 668 & $\sqrt{ }$ \\
\hline $92 w w$ & $\mathrm{~F}$ & B & $\mathrm{J} R$ & 90 & $\mathrm{M}+\mathrm{Na}^{+}$ & 648 & 648 & $\sqrt{ }$ \\
\hline $92 x x$ & G & B & $\mathrm{J} R$ & 61 & $\mathrm{M}+\mathrm{NH}_{4}^{+}$ & 835 & 835 & $\sqrt{ }$ \\
\hline $92 y y$ & A & B & $\mathrm{K} R$ & $>90$ & $\mathrm{M}+\mathrm{Na}^{+}$ & 578 & 578 & $\sqrt{ }$ \\
\hline $92 z z$ & B & $\mathrm{C}$ & $\mathrm{K} R$ & 67 & $\mathrm{M}+\mathrm{Na}^{+}$ & 618 & 618 & $\sqrt{ }$ \\
\hline 92aaa & G & A & $\mathrm{L} R$ & 56 & $\mathrm{M}+\mathrm{NH}_{4}^{+}$ & 789 & 789 & $\sqrt{ }$ \\
\hline 92bbb & $\mathrm{C}$ & $\mathrm{C}$ & $\mathrm{L} R$ & 62 & $\mathrm{M}+\mathrm{Na}^{+}$ & 626 & 626 & $\sqrt{ }$ \\
\hline $92 \mathrm{ccc}$ & G & $\mathrm{C}$ & $\mathrm{M} R$ & 69 & $\mathrm{M}+\mathrm{NH}_{4}^{+}$ & 913 & 913 & $\sqrt{ }$ \\
\hline 92ddd & $\mathrm{C}$ & $\mathrm{C}$ & $\mathrm{M} R$ & 67 & $\mathrm{M}+\mathrm{Na}^{+}$ & 660 & 660 & $\sqrt{ }$ \\
\hline 92eee & B & B & $\mathrm{N} R$ & $>90$ & $\mathrm{M}+\mathrm{Na}^{+}$ & 606 & 606 & $\sqrt{ }$ \\
\hline $92 f f f$ & G & B & $\mathrm{N} R$ & 63 & $\mathrm{M}+\mathrm{NH}_{4}^{+}$ & 915 & 915 & $\sqrt{ }$ \\
\hline 92ggg & G & B & $\mathrm{O} R$ & 36 & $\mathrm{M}+\mathrm{NH}_{4}^{+}$ & 895 & 895 & $\sqrt{ }$ \\
\hline 92hhh & A & $\mathrm{C}$ & $\mathrm{O} R$ & 58 & $\mathrm{M}+\mathrm{Na}^{+}$ & 570 & 570 & $\sqrt{ }$ \\
\hline
\end{tabular}


Step 4. Coupling and tagging of Skeletal Information Element \#2 $\left(\square_{2}\right)$

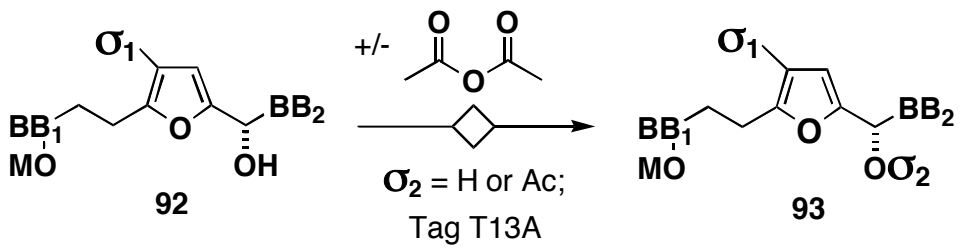

Coupling of $\square_{2}$. The pooled collection of macrobeads 92 from Step $3(2.157 \mathrm{~g}, \sim 0.19 \mathrm{mg} / \mathrm{bead}$, $\sim 11,170$ beads) was then split evenly into two portions (1.08 g each). One portion was subjected to acetylation and the other portion was not. For the acetylation reaction, an oven-dried $120 \mathrm{~mL}$ sealed tube apparatus (ChemGlass) was charged with resin $\mathbf{9 2}$ and flushed with argon for 10 minutes. The resin was then washed with anhydrous $\mathrm{CH}_{2} \mathrm{Cl}_{2}(2 \times 50 \mathrm{~mL} \times 10 \mathrm{~min}$ each $)$ at $\mathrm{rt}$ under argon (washings removed by cannula) and then resuspended in $\mathrm{CH}_{2} \mathrm{Cl}_{2}(55 \mathrm{~mL})$. To this mixture was then added $i$ - $\mathrm{Pr}_{2} \mathrm{NEt}(3.8 \mathrm{~mL}, 0.022 \mathrm{~mol})$, DMAP $(134 \mathrm{mg}, 0.0011 \mathrm{~mol})$, and finally acetic anhydride $(1.04 \mathrm{~mL}, 0.011 \mathrm{~mol})$ with manual agitation of the reaction mixture following each addition. The resulting mixture was sealed under a blanket of argon, the sealed tube was covered with aluminum foil, and the reaction mixture was tumbled at $\mathrm{rt}$ for $28 \mathrm{~h}$. Resin was then isolated by filtration into a $20 \mathrm{~mL}$ polypropylene tube and washed as follows: $5 \mathrm{x} \mathrm{CH}_{2} \mathrm{Cl}_{2}, 5 \mathrm{x}$ THF, $5 \times \mathrm{CH}_{2} \mathrm{Cl}_{2}, \mathrm{CH}_{2} \mathrm{Cl}_{2}$ x $45 \mathrm{~min}, 5 \mathrm{x}$ THF, THF x $45 \mathrm{~min}, 5 \times \mathrm{CH}_{2} \mathrm{Cl}_{2}, \mathrm{CH}_{2} \mathrm{Cl}_{2} \mathrm{x} 45 \mathrm{~min}, 5 \mathrm{x}$ anh. $\mathrm{CH}_{2} \mathrm{Cl}_{2}$, anh. $\mathrm{CH}_{2} \mathrm{Cl}_{2} \times 20 \mathrm{~min}$, and then the solvent was removed under argon flow followed by residual solvent removal in vacuo.

Tagging of $\square_{2}$. The product resin from this acetylation reaction was then added to a freshly prepared solution of tag T13A in $\mathrm{CH}_{2} \mathrm{Cl}_{2}(16.7 \mathrm{~mL}, 4.4 \mathrm{mM})$. The resulting mixture was sealed under an argon cloud and allowed to rotate gently for $1 \mathrm{~h}$. Then, a freshly prepared solution of rhodium triphenylphosphate $(16.66 \mathrm{~mL} ., 4.4 \mathrm{mg} . / \mathrm{mL}$.) was added to the mixture of tags and resin. This vial was then sealed under an argon cloud, capped and sealed with parafilm, wrapped in aluminum foil to prevent exposure to light, and allowed to rotate gently for $15 \mathrm{~h}$. The resin was then isolated by filtration and washed as follows: $2 \times\left(5 \times \mathrm{CH}_{2} \mathrm{Cl}_{2}, \mathrm{CH}_{2} \mathrm{Cl}_{2} \times 15 \mathrm{~min}\right.$.), $3 \times$ ( 5 x THF, THF x 2 h), 5 x anh. THF, anh. anh. THF x 45 min, 5 x anh. $\mathrm{CH}_{2} \mathrm{Cl}_{2}$, anh. $\mathrm{CH}_{2} \mathrm{Cl}_{2} \times 20$ min, and the solvent was removed under argon flow followed by residual solvent removal in vacuo to yield two portions of resin $\mathbf{9 3}$, collectively representing all combinations of $\mathbf{B B}_{1}, \square_{1}$, $\mathbf{B B}_{2}$, and $\square_{2}$, with each combination uniquely encoded with polychlorinated aromatic tags.

Encoding scheme for $\square_{2}$

$\square_{2} \mathbf{A}(\mathrm{H})$

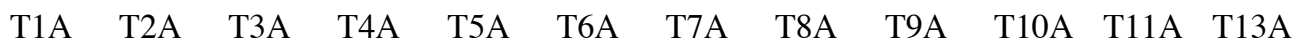

$\square_{2} \mathbf{B}(\mathrm{Ac})$

The compound and chemical tags were then cleaved from 60 individual macrobeads 93 (30 from each portion) and analyzed by LCMS and GC, respectively. These data were found to be consistent for 60/60 (100\%) of these macrobeads, and the compounds cleaved from 55/60 $(92 \%)$ of these macrobeads were determined to be $\geq 70 \%$ pure by LCMS analysis. 
Burke, Berger, and Schreiber - Supporting Information Part A

\section{Results of Step 4}

Structure encoded LCMS analysis by chemical tags $\quad(\square=214 \mathbf{~ n m})$ Purity

$\begin{array}{llllllll}\text { Macrobead } & \mathbf{B B}_{1} & \square_{1} & \mathbf{B B}_{2} & \square_{2} & (\boldsymbol{\%}) & \mathbf{t}_{\mathbf{R}}(\min )\end{array}$
ES+ Mass Spec Ion Calculated Observed

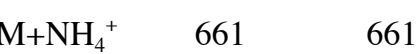

93b $\begin{array}{lllllll}\mathrm{F} & \mathrm{C} & \mathrm{H} S & \mathrm{~A} & >90 & 9.36\end{array}$

93c

93d

93e

$93 f$

93

93h

93

93

$93 k$

931

93m

93n

930

93p

93q

93

93

93

93u

$93 \mathrm{v}$

93w

93x

$93 y$

$\mathrm{A} \mathrm{M} R \quad \mathrm{~A}=70$

$\begin{array}{llllll}93 a a & \mathrm{~B} & \mathrm{C} & \mathrm{K} R & \mathrm{~A} & <70\end{array}$

$\begin{array}{llllll}93 \mathrm{bb} & \mathrm{G} & \mathrm{C} & \mathrm{H} S & \mathrm{~A} & 88\end{array}$

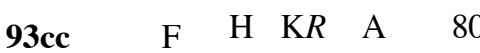

93dd A $\quad \mathrm{C} \quad \mathrm{OS}$ A 68

93ee $\mathrm{E} \quad \mathrm{B} \quad \mathrm{H} R \quad \mathrm{~B} \quad>90$

$\begin{array}{llllll}\text { 93ff } & \mathrm{F} & \mathrm{C} & \mathrm{N} R & \mathrm{~B} & 85\end{array}$

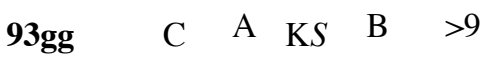

$\begin{array}{llllll}93 \mathrm{hh} & \mathrm{B} & \mathrm{B} & \mathrm{O} S & \mathrm{~B} & >70\end{array}$

$\begin{array}{llllll}\text { 93ii } & \mathrm{E} & \mathrm{B} & \mathrm{O} R & \mathrm{~B} & 69\end{array}$

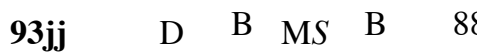

$\begin{array}{llllll}93 \mathrm{kk} & \mathrm{C} & \mathrm{A} & \mathrm{J} R & \mathrm{~B} & >90\end{array}$

93II
$\begin{array}{lllll}\mathrm{G} & \mathrm{A} & \mathrm{I} S & \mathrm{~B} & >90\end{array}$

9.36
11.89

8.15

10.10

9.88

6.98

9.12

11.54

9.55

6.70

9.08

5.43

9.14

7.15

11.06

8.43

8.07

10.37

7.99

8.97

6.59

8.79

8.76

6.66

$$
10.79
$$

10.79

10.34

10.44

10.74

9.48

9.83

7.50

12.59

11.76

10.85

9.56

9.35

10.28

10.71
$\mathrm{M}+\mathrm{NH}_{4}{ }^{+} \quad 915$

$\mathrm{M}+\mathrm{NH}_{4}{ }^{+} \quad 525$

$\mathrm{M}+\mathrm{NH}_{4}{ }^{+} \quad 639$

$\mathrm{M}+\mathrm{NH}_{4}{ }^{+} \quad 565$

$\mathrm{M}+\mathrm{Na}^{+} \quad 454$

$\mathrm{M}+\mathrm{NH}_{4}{ }^{+} \quad 499$

$\mathrm{M}+\mathrm{Na}^{+} \quad 662$

$\mathrm{M}+\mathrm{Na}^{+} \quad 508$

$\mathrm{M}+\mathrm{NH}_{4}{ }^{+} \quad 493$

$\mathrm{M}+\mathrm{NH}_{4}{ }^{+} \quad 597$

$\mathrm{M}+\mathrm{NH}_{4}{ }^{+} \quad 489$

$\mathrm{M}+\mathrm{Na}^{+} \quad 460$

$\mathrm{M}+\mathrm{NH}_{4}{ }^{+} \quad 537$

$\mathrm{M}+\mathrm{NH}_{4}{ }^{+} \quad 839$

$\mathrm{M}+\mathrm{Na}^{+} \quad 654$

$\mathrm{M}+\mathrm{Na}^{+} \quad 480$

$\mathrm{M}+\mathrm{NH}_{4}{ }^{+} \quad 835$

$\mathrm{M}+\mathrm{Na}^{+} \quad 452$

$\mathrm{M}+\mathrm{NH}_{4}{ }^{+} \quad 573$

$\mathrm{M}+\mathrm{Na}^{+} \quad 484$

$\mathrm{M}+\mathrm{NH}_{4}{ }^{+} \quad 553$

$\mathrm{M}+\mathrm{Na}^{+} \quad 604$

$\mathrm{M}+\mathrm{NH}_{4}{ }^{+} \quad 493$

$\mathrm{M}+\mathrm{Na}^{+} \quad 564$

$\mathrm{M}+\mathrm{Na}^{+} \quad 570$

$\mathrm{M}+\mathrm{NH}_{4}{ }^{+} \quad 613$

$\mathrm{M}+\mathrm{NH}_{4}{ }^{+} \quad 853$

$\mathrm{M}+\mathrm{NH}_{4}{ }^{+} \quad 645$

$\mathrm{M}+\mathrm{NH}_{4}{ }^{+} \quad 565$

$\mathrm{M}+\mathrm{NH}_{4}{ }^{+} \quad 615$

$\mathrm{M}+\mathrm{NH}_{4}{ }^{+} \quad 777$

$\mathrm{M}+\mathrm{NH}_{4}{ }^{+} \quad 621$

$\mathrm{M}+\mathrm{NH}_{4}{ }^{+} \quad 623$

$\mathrm{M}+\mathrm{NH}_{4}{ }^{+} \quad 669$

$\mathrm{M}+\mathrm{NH}_{4}{ }^{+} \quad 631$

$\mathrm{M}+\mathrm{NH}_{4}{ }^{+} \quad 541$

$\mathrm{M}+\mathrm{NH}_{4}{ }^{+} \quad 819$
Mass spec

consistent with tag-encoded structure

915

525

639

565

454

499

662

508

493

597

489

460

537

839

654

480

835

452

573

484

553

604

493

564

570

613

853

645

565

615

777

621

623

669

631

541

819

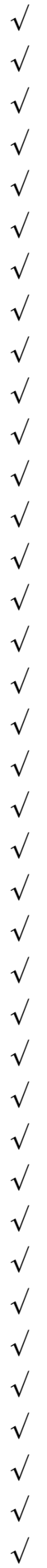


Burke, Berger, and Schreiber - Supporting Information Part A

\begin{tabular}{|c|c|c|c|c|c|c|c|c|c|c|}
\hline Macrobead & $\mathbf{B B}_{1}$ & $\square_{1}$ & $\mathbf{B B}_{2}$ & $\square_{2}$ & $\begin{array}{c}\text { Purity } \\
(\%)\end{array}$ & $\mathbf{t}_{\mathbf{R}}(\min )$ & Ion & Calculated & Observed & $\begin{array}{l}\text { Tag-encoded } \\
\text { structure }\end{array}$ \\
\hline 93mm & B & $A$ & $\mathrm{M} S$ & B & 90 & 9.57 & $\mathrm{M}+\mathrm{NH}_{4}^{+}$ & 551 & 551 & $\sqrt{ }$ \\
\hline 93nn & G & B & $\mathrm{J} R$ & B & 71 & 11.43 & $\mathrm{M}+\mathrm{NH}_{4}^{+}$ & 877 & 877 & $\sqrt{ }$ \\
\hline 9300 & A & B & $\mathrm{A} R$ & B & $>90$ & 8.95 & $\mathrm{M}+\mathrm{NH}_{4}^{+}$ & 539 & 539 & $\sqrt{ }$ \\
\hline 93pp & A & $\mathrm{C}$ & GS & B & 77 & 8.78 & $\mathrm{M}+\mathrm{NH}_{4}^{+}$ & 519 & 519 & $\sqrt{ }$ \\
\hline 93qq & A & $\mathrm{C}$ & $\mathrm{N} R$ & B & 88 & 11.27 & $\mathrm{M}+\mathrm{NH}_{4}^{+}$ & 627 & 627 & $\sqrt{ }$ \\
\hline 93rr & B & $\mathrm{C}$ & $\mathrm{J} S$ & B & $>90$ & 10.07 & $\mathrm{M}+\mathrm{NH}_{4}^{+}$ & 575 & 575 & $\sqrt{ }$ \\
\hline 93ss & B & A & $\mathrm{J} S$ & B & $>90$ & 8.27 & $\mathrm{M}+\mathrm{NH}_{4}^{+}$ & 485 & 485 & $\sqrt{ }$ \\
\hline 93tt & B & $\mathrm{C}$ & $\mathrm{F} R$ & B & $>90$ & 9.88 & $\mathrm{M}+\mathrm{NH}_{4}^{+}$ & 595 & 595 & $\sqrt{ }$ \\
\hline 93uu & A & B & $\mathrm{M} R$ & B & $>90$ & 9.66 & $\mathrm{M}+\mathrm{NH}_{4}^{+}$ & 601 & 601 & $\sqrt{ }$ \\
\hline $93 v v$ & B & A & $\mathrm{OS}$ & B & $>90$ & 9.95 & $\mathrm{M}+\mathrm{NH}_{4}^{+}$ & 545 & 545 & $\sqrt{ }$ \\
\hline 93ww & $\mathrm{C}$ & A & MS & B & $>90$ & 11.31 & $\mathrm{M}+\mathrm{NH}_{4}^{+}$ & 607 & 607 & $\sqrt{ }$ \\
\hline $93 \times x$ & A & $\mathrm{C}$ & $\mathrm{J} S$ & B & $>90$ & 9.39 & $\mathrm{M}+\mathrm{NH}_{4}^{+}$ & 547 & 547 & $\sqrt{ }$ \\
\hline $93 y y$ & D & A & $\mathrm{J} S$ & B & $>90$ & 7.01 & $\mathrm{M}+\mathrm{NH}_{4}^{+}$ & 487 & 487 & $\sqrt{ }$ \\
\hline 93zz & A & $\mathrm{C}$ & $\mathrm{A} S$ & B & $>90$ & 9.87 & $\mathrm{M}+\mathrm{NH}_{4}^{+}$ & 551 & 551 & $\sqrt{ }$ \\
\hline 93aаa & D & A & $\mathrm{H} R$ & B & $>90$ & 6.62 & $\mathrm{M}+\mathrm{NH}_{4}^{+}$ & 493 & 493 & $\sqrt{ }$ \\
\hline 93bbb & $\mathrm{E}$ & $\mathrm{C}$ & $\mathrm{H} R$ & B & $>90$ & 8.56 & $\mathrm{M}+\mathrm{NH}_{4}^{+}$ & 627 & 627 & $\sqrt{ }$ \\
\hline $93 \mathrm{ccc}$ & $\mathrm{C}$ & B & $\mathrm{CS}$ & B & $<70$ & 11.12 & $\mathrm{M}+\mathrm{Na}^{+}$ & 614 & 614 & $\sqrt{ }$ \\
\hline 93ddd & $\mathrm{F}$ & B & $\mathrm{L} S$ & B & 73 & 10.83 & $\mathrm{M}+\mathrm{NH}_{4}^{+}$ & 717 & 717 & $\sqrt{ }$ \\
\hline 93eee & D & B & $\mathrm{L} S$ & B & $>90$ & 9.20 & $\mathrm{M}+\mathrm{NH}_{4}{ }^{+}$ & 597 & 597 & $\sqrt{ }$ \\
\hline 93fff & A & A & $\mathrm{E} S$ & B & $>90$ & 8.06 & $\mathrm{M}+\mathrm{Na}^{+}$ & 446 & 446 & $\sqrt{ }$ \\
\hline 93ggg & D & A & $\mathrm{E} S$ & B & $>90$ & 7.71 & $\mathrm{M}+\mathrm{NH}_{4}^{+}$ & 471 & 471 & $\sqrt{ }$ \\
\hline 93hhh & $\mathrm{D}$ & $\mathrm{C}$ & $\mathrm{J} R$ & B & 87 & 9.08 & $\mathrm{M}+\mathrm{NH}_{4}^{+}$ & 577 & 577 & $\sqrt{ }$ \\
\hline
\end{tabular}

$426.5 \mathrm{mg}$ of each of the two portions of light brown product resin 93 ( 4410 macrobeads, multiplicative factor $=3.5)$, representing all possible combinations of $\mathbf{B B}_{1}, \square_{1}, \mathbf{B B}_{2}$, and $\square_{2}$ in both enantiomeric and diasteromeric forms, were then pooled together in a single polypropylene tube and well mixed. 
Step 5. NBS and PPTS-mediated folding process

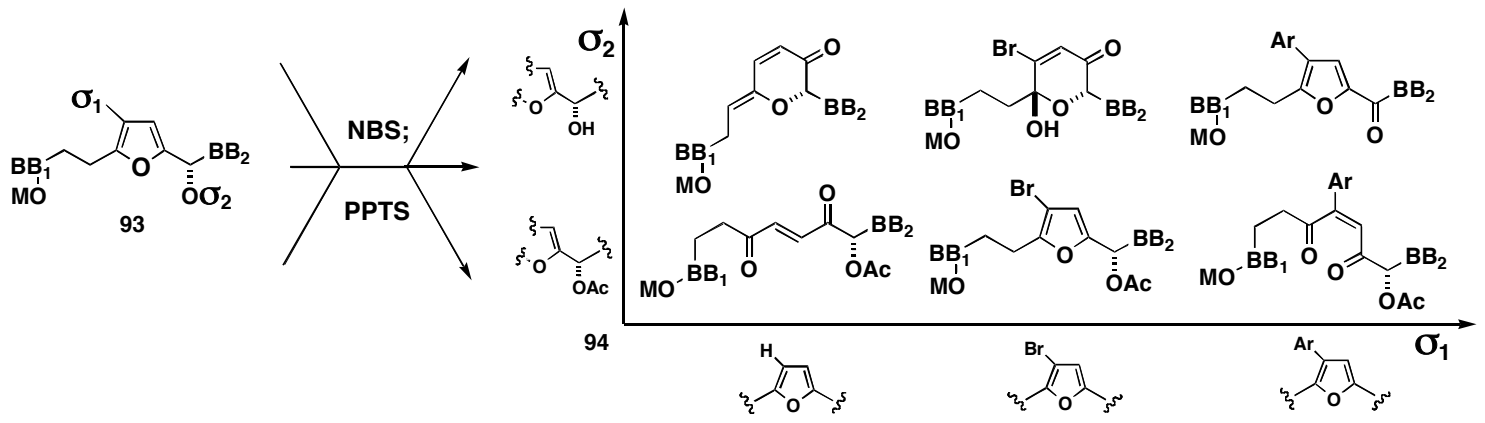

Folding process. A $120 \mathrm{~mL}$ sealed tube apparatus (Chemglass) was charged with THF (64 mL), $\mathrm{H}_{2} \mathrm{O}$ (16 mL, THF and $\mathrm{H}_{2} \mathrm{O}$ were mixed to homogeneity), and macrobead-bound substrates 93 (853 mg, $\sim 5.2$ macrobeads $/ \mathrm{mg}, \sim 4410$ macrobeads, multiplicative factor $=3.5$; substrate macrobeads were light brown) at rt under ambient. The resulting mixture was agitated manually for $2 \mathrm{~min}$ and then let stand at $\mathrm{rt}$ under ambient for 10 minutes. To this mixture was then added $\mathrm{NaHCO}_{3}(3.06 \mathrm{~g}, 36 \mathrm{mmol})$ and $\mathrm{NaOAc}(1.48 \mathrm{~g}, 18 \mathrm{mmol})$ and the resulting mixture let stand at $\mathrm{rt}$ for 10 minutes with periodic manual agitation (2 layers formed). To this mixture was then added NBS (2.136 g, $12 \mathrm{mmol})$ and the resulting yellow-tinted reaction mixture was sealed under ambient and manually agitated. The flask was then wrapped in aluminum foil and tumbled at $\mathrm{rt}$ for $1 \mathrm{~h}$ (the reaction solution turned dark yellow/yellow-orange). The resin was then isolated by filtration into a $20 \mathrm{~mL}$ polypropylene tube using THF and $\mathrm{H}_{2} \mathrm{O}$ (macrobeads were light yellow) and then washed as follows: 5 x THF, $5 \times \mathrm{H}_{2} \mathrm{O}, 5 \times \mathrm{THF}$, THF: $\mathrm{H}_{2} \mathrm{O} 3: 1 \times 1 \mathrm{~h}, 5 \times \mathrm{THF}$, THF $\times 1$ h, $5 \times \mathrm{CH}_{2} \mathrm{Cl}_{2}, \mathrm{CH}_{2} \mathrm{Cl}_{2} \times 30$ min, 5 x anhydrous $\mathrm{CH}_{2} \mathrm{Cl}_{2}$, anhydrous $\mathrm{CH}_{2} \mathrm{Cl}_{2} \times 30$ min, 5 x anhydrous $\mathrm{CH}_{2} \mathrm{Cl}_{2} \times 2$ min each, and then the solvent was removed under argon flow followed by residual solvent removal in vacuo $(\sim 1 \mathrm{~h})$. An oven-dried, $350 \mathrm{~mL}$ sealed tube apparatus (Chemglass) was then charged at $\mathrm{rt}$ under a cloud of argon with anhydrous $\mathrm{CH}_{2} \mathrm{Cl}_{2}(200 \mathrm{~mL})$ and pyridinium $p$-toluenesulfonate $\left(37.7 \mathrm{mg}, 0.15 \mathrm{mmol}, 0.00075 \mathrm{M}\right.$ in $\left.\mathrm{CH}_{2} \mathrm{Cl}_{2}\right)$. The flask was then sealed under a cloud of argon and manually agitated to make a clear, colorless solution. The resin was added, the flask was again sealed under a cloud of argon, and the reaction mixture was warmed to $40-45^{\circ} \mathrm{C}$ (oil bath) and maintained at that temperature for $20 \mathrm{~h}$ with periodic manual agitation every 4-8 h. Resin was then isolated by filtration into a $20 \mathrm{~mL}$ polypropylene tube (using THF and a glass funnel to transfer resin) and then washed as follows: $5 \mathrm{x}$ THF, $5 \mathrm{x} \mathrm{H}_{2} \mathrm{O}, 5$ x THF, THF:dilute aq. $\mathrm{NaHCO}_{3}$ (sat. aq. $\mathrm{NaHCO}_{3}: \mathrm{H}_{2} \mathrm{O}$ 1:2) 1:1 x 1 h, 5 x THF, 5 x $\mathrm{H}_{2} \mathrm{O}, 5$ x THF, THF:dilute aq. $\mathrm{NH}_{4} \mathrm{Cl}$ (sat. aq. $\mathrm{NH}_{4} \mathrm{Cl}: \mathrm{H}_{2} \mathrm{O}$ 1:2) 1:1 x $1 \mathrm{~h}, 5$ x THF, 5 x $\mathrm{H}_{2} \mathrm{O}, 5$ x THF, THF: $\mathrm{H}_{2} \mathrm{O} 3: 1 \times 45 \mathrm{~min}, 5 \mathrm{x}$ THF, THF x $45 \mathrm{~min}, 5 \times \mathrm{CH}_{2} \mathrm{Cl}_{2}, \mathrm{CH}_{2} \mathrm{Cl}_{2}$ x 30 min, 5 x anh. $\mathrm{CH}_{2} \mathrm{Cl}_{2}$, anh. $\mathrm{CH}_{2} \mathrm{Cl}_{2} \times 30 \mathrm{~min}$, and $5 \mathrm{x}$ anhydrous $\mathrm{CH}_{2} \mathrm{Cl}_{2} \times 2$ min each. Solvent was then removed under argon flow followed by residual solvent removal in vacuo to yield a collection of macrobeadbound products 94 representing a complete, combinatorial $(3 \times 2=6)$ matrix of molecular skeletons, each derivatized with a complete, combinatorial $(7 \times 15=105)$ matrix of building blocks in both enantiomeric and diastereomeric forms $(6 \times 15 \times 2=1260)$.

The compound and chemical tags were cleaved from 120 individual product macrobeads 94 and then analyzed by LCMS and GC, respectively. The LCMS data were consistent with the formation of the functionalized skeleton encoded by the corresponding chemical tags in 120 out of 120 cases (100\%). Moreover, 84/120 (70\%) of these compounds were determined to be $\geq$ $70 \%$ pure by LCMS analysis. These results are summarized in tabular format below. 
Burke, Berger, and Schreiber - Supporting Information Part A

\section{Results of step 5}

\begin{tabular}{|c|c|c|c|c|c|c|c|c|c|}
\hline \multirow[b]{2}{*}{ Macrobead } & \multicolumn{3}{|c|}{$\begin{array}{c}\text { Structure } \\
\text { encoded by } \\
\text { chemical tags }\end{array}$} & \multicolumn{2}{|c|}{$\begin{array}{c}\text { LCMS analysis } \\
(\square=214 \mathrm{~nm})\end{array}$} & \multicolumn{3}{|c|}{ ES+ Mass Spec } & \multirow{2}{*}{$\begin{array}{c}\text { Mass Spec consistent } \\
\text { with } \\
\text { tag-encoded } \\
\text { structure }\end{array}$} \\
\hline & $\mathbf{B B}_{1}$ & $\square_{1} \mathbf{B B}_{2}$ & $\square_{2}$ & $\begin{array}{l}\text { Purity } \\
(\%)\end{array}$ & $\begin{array}{c}\mathbf{t}_{\mathbf{R}} \\
(\mathbf{m i n})\end{array}$ & Ion & Calculated & Observed & \\
\hline $94 a$ & $\mathrm{~F}$ & $\mathrm{~B} \quad \mathrm{D} R$ & B & 37 & 10.61 & $\mathrm{M}+\mathrm{NH}_{4}^{+}$ & 689 & 689 & $\sqrt{ }$ \\
\hline 94b & $\mathrm{E}$ & $\mathrm{A} \quad \mathrm{A} R$ & B & $>90$ & 6.98 & $\mathrm{M}+\mathrm{NH}_{4}^{+}$ & 551 & 551 & $\sqrt{ }$ \\
\hline $94 c$ & B & C $\mathrm{K} S$ & B & 65 & 10.39 & $\mathrm{M}+\mathrm{NH}_{4}^{+}$ & 671 & 671 & $\sqrt{ }$ \\
\hline 94d & $\mathrm{D}$ & A $\mathrm{J} R$ & A & 21 & 6.06 & $\mathrm{M}+\mathrm{H}^{+}$ & 426 & 426 & $\sqrt{ }$ \\
\hline $94 e$ & $\mathrm{E}$ & A $F R$ & B & $>90$ & 6.38 & $\mathrm{M}+\mathrm{NH}_{4}^{+}$ & 567 & 567 & $\sqrt{ }$ \\
\hline $94 f$ & $\mathrm{G}$ & $\mathrm{B} \mathrm{D} S$ & A & 49 & 10.98 & $\mathrm{M}+\mathrm{NH}_{4}^{+}$ & 855 & 855 & $\sqrt{ }$ \\
\hline $94 \mathrm{~g}$ & $\mathrm{D}$ & A $\mathrm{L} S$ & $\mathrm{~B}$ & $>90$ & 7.75 & $\mathrm{M}+\mathrm{NH}_{4}^{+}$ & 535 & 535 & $\sqrt{ }$ \\
\hline 94h & B & $\mathrm{A} \quad \mathrm{I} R$ & B & $>90$ & 7.78 & $\mathrm{M}+\mathrm{NH}_{4}^{+}$ & 521 & 521 & $\sqrt{ }$ \\
\hline $94 \mathbf{i}$ & B & $\mathrm{A} \quad \mathrm{I} R$ & A & $>90$ & 7.67 & $\mathrm{M}+\mathrm{H}^{+}$ & 444 & 444 & $\sqrt{ }$ \\
\hline $94 j$ & $\mathrm{~F}$ & $\mathrm{C} \quad \mathrm{OR}$ & B & 43 & 11.43 & $\mathrm{M}+\mathrm{NH}_{4}^{+}$ & 773 & 773 & $\sqrt{ }$ \\
\hline $94 k$ & $\mathrm{G}$ & A $\quad F R$ & A & 82 & 9.91 & $\mathrm{M}+\mathrm{NH}_{4}^{+}$ & 775 & 775 & $\sqrt{ }$ \\
\hline 941 & $\mathrm{D}$ & $\mathrm{B} \quad \mathrm{M} R$ & A & 79 & 8.06 & $\mathrm{M}+\mathrm{NH}_{4}^{+}$ & 605 & 605 & $\sqrt{ }$ \\
\hline $94 m$ & B & $\mathrm{B} \quad \mathrm{B} R$ & A & $>90$ & 7.45 & $\mathrm{M}+\mathrm{Na}^{+}$ & 498 & 498 & $\sqrt{ }$ \\
\hline $94 n$ & A & B $\mathrm{FS}$ & B & $>90$ & 8.31 & $\mathrm{M}+\mathrm{Na}^{+}$ & 560 & 560 & $\sqrt{ }$ \\
\hline 940 & $\mathrm{C}$ & B $\mathrm{N} S$ & A & $<70$ & 11.7 & $\mathrm{M}+\mathrm{Na}^{+}$ & 678 & 678 & $\sqrt{ }$ \\
\hline $94 p$ & $\mathrm{G}$ & В $\mathrm{M} S$ & A & 47 & 11.32 & $\mathrm{M}+\mathrm{NH}_{4}^{+}$ & 917 & 917 & $\sqrt{ }$ \\
\hline $94 q$ & $\mathrm{~F}$ & $\mathrm{~B} \quad \mathrm{~N} R$ & A & 74 & 10.32 & $\mathrm{M}+\mathrm{NH}_{4}^{+}$ & 739 & 739 & $\sqrt{ }$ \\
\hline $94 r$ & B & $\mathrm{A} \quad \mathrm{C} R$ & A & $>90$ & 7.73 & $\mathrm{M}+\mathrm{H}^{+}$ & 414 & 414 & $\sqrt{ }$ \\
\hline 94s & B & A $\mathrm{FS}$ & A & $>90$ & 7.26 & $\mathrm{M}+\mathrm{H}^{+}$ & 444 & 444 & $\sqrt{ }$ \\
\hline $94 t$ & G & $\mathrm{A} \quad \mathrm{B} R$ & A & 68 & 10.10 & $\mathrm{M}+\mathrm{NH}_{4}^{+}$ & 711 & 711 & $\sqrt{ }$ \\
\hline 94u & D & C $\mathrm{H} S$ & B & 71 & 7.81 & $\mathrm{M}+\mathrm{H}^{+}$ & 582 & 582 & $\sqrt{ }$ \\
\hline $94 v$ & $\mathrm{D}$ & $\mathrm{C} \quad \mathrm{E} R$ & A & 54 & 9.28 & $\mathrm{M}+\mathrm{H}^{+}$ & 500 & 500 & $\sqrt{ }$ \\
\hline $94 w$ & A & В $\mathrm{K} S$ & $\mathrm{~B}$ & $>90$ & 9.99 & $\mathrm{M}+\mathrm{NH}_{4}^{+}$ & 615 & 615 & $\sqrt{ }$ \\
\hline $94 x$ & A & C $\mathrm{H} S$ & A & 49 & 8.76 & $\mathrm{M}+\mathrm{H}^{+}$ & 492 & 492 & $\sqrt{ }$ \\
\hline $94 y$ & B & $\mathrm{C} \quad \mathrm{L} R$ & B & 32 & 10.12 & $\mathrm{M}+\mathrm{NH}_{4}^{+}$ & 623 & 623 & $\sqrt{ }$ \\
\hline $94 z$ & $\mathrm{E}$ & B $\mathrm{C} S$ & A & $>90$ & 6.58 & $\mathrm{M}+\mathrm{NH}_{4}^{+}$ & 573 & 573 & $\sqrt{ }$ \\
\hline 94aa & B & $\mathrm{B} \quad \mathrm{K} R$ & B & $>90$ & 10.63 & $\mathrm{M}+\mathrm{NH}_{4}^{+}$ & 643 & 643 & $\sqrt{ }$ \\
\hline $94 \mathrm{bb}$ & $\mathrm{C}$ & A $C R$ & A & $>90$ & 9.72 & $\mathrm{M}+\mathrm{H}^{+}$ & 470 & 470 & $\sqrt{ }$ \\
\hline $94 \mathrm{cc}$ & $\mathrm{E}$ & A $\mathrm{J} R$ & A & 51 & 6.06 & $\mathrm{M}+\mathrm{H}^{+}$ & 470 & 470 & $\sqrt{ }$ \\
\hline 94dd & $\mathrm{F}$ & A $\mathrm{G} S$ & A & 60 & 7.54 & $\mathrm{M}+\mathrm{H}^{+}$ & 518 & 518 & $\sqrt{ }$ \\
\hline 94ee & B & A $\quad \mathrm{N} R$ & A & $>90$ & 9.74 & $\mathrm{M}+\mathrm{H}^{+}$ & 504 & 504 & $\sqrt{ }$ \\
\hline 94ff & A & C $\mathrm{OS}$ & A & 3 & 10.73 & $\mathrm{M}+\mathrm{H}^{+}$ & 546 & 546 & $\sqrt{ }$ \\
\hline 94gg & B & A $\quad \mathrm{L} S$ & A & $>90$ & 8.81 & $\mathrm{M}+\mathrm{H}^{+}$ & 456 & 456 & $\sqrt{ }$ \\
\hline $94 \mathrm{hh}$ & $\mathrm{C}$ & A $\quad \mathrm{F} R$ & B & $>90$ & 9.26 & $\mathrm{M}+\mathrm{NH}_{4}^{+}$ & 577 & 577 & $\sqrt{ }$ \\
\hline 94ii & B & A $\mathrm{FS}$ & A & $>90$ & 7.27 & $\mathrm{M}+\mathrm{H}^{+}$ & 444 & 444 & $\sqrt{ }$ \\
\hline 94jj & $\mathrm{C}$ & В $\mathrm{OS}$ & A & $>90$ & 11.71 & $\mathrm{M}+\mathrm{Na}^{+}$ & 658 & 658 & $\sqrt{ }$ \\
\hline $94 \mathrm{kk}$ & G & $\mathrm{B} \quad \mathrm{F} R$ & A & 65 & 10.07 & $\mathrm{M}+\mathrm{NH}_{4}^{+}$ & 871 & 871 & $\sqrt{ }$ \\
\hline 9411 & A & C NS & A & 84 & 10.86 & $\mathrm{M}+\mathrm{H}^{+}$ & 566 & 566 & $\sqrt{ }$ \\
\hline
\end{tabular}


Burke, Berger, and Schreiber - Supporting Information Part A

\begin{tabular}{|c|c|c|c|c|c|c|c|c|c|c|}
\hline Macrobead & $\mathbf{B B}_{1}$ & $\square_{1}$ & $\mathbf{B B}_{2}$ & $\square_{2}$ & $\begin{array}{c}\text { Purity } \\
\text { (\%) }\end{array}$ & $\underset{(\mathbf{m i n})}{\stackrel{\mathbf{t}_{\mathbf{R}}}{ }}$ & Ion & Calculated & Observed & $\begin{array}{l}\text { Tag-encoded } \\
\text { structure }\end{array}$ \\
\hline 94mm & $\mathrm{D}$ & $\mathrm{B}$ & IS & A & 78 & 6.69 & $\mathrm{M}+\mathrm{NH}_{4}^{+}$ & 559 & 559 & $\sqrt{ }$ \\
\hline $94 n n$ & E & B & $\mathrm{I} R$ & B & $<70$ & 8.12 & $\mathrm{M}+\mathrm{NH}_{4}^{+}$ & 629 & 629 & $\sqrt{ }$ \\
\hline 9400 & A & B & $\mathrm{N} R$ & B & $>90$ & 10.36 & $\mathrm{M}+\mathrm{NH}_{4}^{+}$ & 615 & 615 & $\sqrt{ }$ \\
\hline $94 p p$ & E & B & $\mathrm{L} S$ & A & 87 & 7.75 & $\mathrm{M}+\mathrm{NH}_{4}^{+}$ & 615 & 615 & $\sqrt{ }$ \\
\hline 94qq & B & B & $\mathrm{L} S$ & B & $>90$ & 10.22 & $\mathrm{M}+\mathrm{Na}^{+}$ & 600 & 600 & $\sqrt{ }$ \\
\hline 94rr & F & B & $\mathrm{B} S$ & A & 64 & 8.38 & $\mathrm{M}+\mathrm{NH}_{4}^{+}$ & 615 & 615 & $\sqrt{ }$ \\
\hline 94ss & $\mathrm{C}$ & A & $\mathrm{D} S$ & A & $>90$ & 10.54 & $\mathrm{M}+\mathrm{H}^{+}$ & 484 & 484 & $\sqrt{ }$ \\
\hline 94tt & $\mathrm{C}$ & A & $\mathrm{E} R$ & B & $>70$ & 10.14 & $\mathrm{M}+\mathrm{H}^{+}$ & 524 & 524 & $\sqrt{ }$ \\
\hline $94 u u=49 !$ & B & $\mathrm{C}$ & $\mathrm{A} S$ & A & $>90$ & 10.06 & $\mathrm{M}+\mathrm{H}^{+}$ & 518 & 518 & $\sqrt{ }$ \\
\hline $94 v v$ & D & $\mathrm{C}$ & $\mathrm{L} S$ & A & $>90$ & 9.87 & $\mathrm{M}+\mathrm{H}^{+}$ & 548 & 548 & $\sqrt{ }$ \\
\hline 94ww & F & A & $\mathrm{H} R$ & B & $>90$ & 7.98 & $\mathrm{M}+\mathrm{NH}_{4}^{+}$ & 629 & 629 & $\sqrt{ }$ \\
\hline $\mathbf{9 4 x x}$ & G & $\mathrm{C}$ & $\mathrm{L} S$ & A & ND & $>12.5$ & $\mathrm{M}+\mathrm{NH}_{4}{ }^{+}$ & 878 & 878 & $\sqrt{ }$ \\
\hline 94yy & $\mathrm{C}$ & $\mathrm{C}$ & $\mathrm{J} S$ & B & 49 & 10.92 & $\mathrm{M}+\mathrm{H}^{+}$ & 630 & 630 & $\sqrt{ }$ \\
\hline $94 z z$ & E & B & $\mathrm{H} R$ & B & $>70$ & 7.51 & $\mathrm{M}+\mathrm{NH}_{4}^{+}$ & 615 & 615 & $\sqrt{ }$ \\
\hline 94aaa & E & $\mathrm{C}$ & $\mathrm{A} R$ & A & 79 & 8.96 & $\mathrm{M}+\mathrm{H}^{+}$ & 564 & 564 & $\sqrt{ }$ \\
\hline 94bbb & A & B & $\mathrm{M} S$ & A & $>70$ & 8.24 & $\mathrm{M}+\mathrm{Na}^{+}$ & 580 & 580 & $\sqrt{ }$ \\
\hline $94 \mathrm{ccc}$ & D & $\mathrm{C}$ & $\mathrm{L} R$ & A & 61 & 9.83 & $\mathrm{M}+\mathrm{H}^{+}$ & 548 & 548 & $\sqrt{ }$ \\
\hline 94ddd & $\mathrm{C}$ & $\mathrm{A}$ & IS & A & $>90$ & 9.40 & $\mathrm{M}+\mathrm{H}^{+}$ & 500 & 500 & $\sqrt{ }$ \\
\hline 94eee & D & A & $\mathrm{H} S$ & B & $>90$ & 5.97 & $\mathrm{M}+\mathrm{NH}_{4}^{+}$ & 509 & 509 & $\sqrt{ }$ \\
\hline $94 \mathrm{fff}$ & B & A & $\mathrm{L} R$ & B & $>70$ & 8.69 & $\mathrm{M}+\mathrm{Na}^{+}$ & 538 & 538 & $\sqrt{ }$ \\
\hline 94ggg & A & $\mathrm{C}$ & $\mathrm{G} R$ & B & 41 & 8.95 & $\mathrm{M}+\mathrm{Na}^{+}$ & 540 & 540 & $\sqrt{ }$ \\
\hline 94hhh & F & A & $\mathrm{J} R$ & A & 43 & 8.38 & $\mathrm{M}+\mathrm{H}^{+}$ & 546 & 546 & $\sqrt{ }$ \\
\hline 94iii & A & $\mathrm{C}$ & $\mathrm{N} R$ & A & $>90$ & 10.95 & $\mathrm{M}+\mathrm{H}^{+}$ & 566 & 566 & $\sqrt{ }$ \\
\hline 94jjjj & A & $\mathrm{C}$ & $\mathrm{L} S$ & A & $>90$ & 10.10 & $\mathrm{M}+\mathrm{H}^{+}$ & 518 & 518 & $\sqrt{ }$ \\
\hline 94kkk & B & A & $\mathrm{A} R$ & B & $>90$ & 8.04 & $\mathrm{M}+\mathrm{NH}_{4}^{+}$ & 505 & 505 & $\sqrt{ }$ \\
\hline $94 I I I$ & $\mathrm{C}$ & $\mathrm{C}$ & $\mathrm{M} S$ & B & ND & 12.07 & $\mathrm{M}+\mathrm{NH}_{4}{ }^{+}$ & 713 & 713 & $\sqrt{ }$ \\
\hline $94 \mathrm{mmm}$ & $\mathrm{E}$ & $\mathrm{C}$ & $\mathrm{A} S$ & A & 78 & 8.97 & $\mathrm{M}+\mathrm{H}^{+}$ & 564 & 564 & $\sqrt{ }$ \\
\hline 94nnn & $\mathrm{C}$ & $\mathrm{C}$ & $\mathrm{N} S$ & A & ND & $>12.5$ & $\mathrm{M}+\mathrm{H}^{+}$ & 650 & 650 & $\sqrt{ }$ \\
\hline 94000 & $\mathrm{C}$ & B & $\mathrm{D} R$ & A & $>90$ & 10.60 & $\mathrm{M}+\mathrm{Na}^{+}$ & 602 & 602 & $\sqrt{ }$ \\
\hline 94ppp & B & B & $\mathrm{G} R$ & B & $>90$ & 8.55 & $\mathrm{M}+\mathrm{Na}^{+}$ & 540 & 540 & $\sqrt{ }$ \\
\hline 94qqq & $\mathrm{F}$ & A & $\mathrm{M} R$ & A & 70 & 9.63 & $\mathrm{M}+\mathrm{H}^{+}$ & 612 & 612 & $\sqrt{ }$ \\
\hline 94rrr & A & A & $\mathrm{L} R$ & A & $>90$ & 7.98 & $\mathrm{M}+\mathrm{H}^{+}$ & 428 & 428 & $\sqrt{ }$ \\
\hline 94sss & E & A & $\mathrm{OS}$ & A & 81 & 8.30 & $\mathrm{M}+\mathrm{H}^{+}$ & 530 & 530 & $\sqrt{ }$ \\
\hline 94ttt & $\mathrm{E}$ & $\mathrm{C}$ & $\mathrm{B} R$ & A & 72 & 8.41 & $\mathrm{M}+\mathrm{H}^{+}$ & 516 & 516 & $\sqrt{ }$ \\
\hline 94uuu & G & B & $\mathrm{N} S$ & A & 37 & 12.08 & $\mathrm{M}+\mathrm{NH}_{4}^{+}$ & 931 & 931 & $\sqrt{ }$ \\
\hline $94 v v v$ & $\mathrm{~F}$ & $\mathrm{C}$ & $\mathrm{K} R$ & A & 82 & 11.78 & $\mathrm{M}+\mathrm{H}^{+}$ & 716 & 716 & $\sqrt{ }$ \\
\hline 94www & A & B & $\mathrm{M} S$ & A & $>90$ & 8.24 & $\mathrm{M}+\mathrm{Na}^{+}$ & 580 & 580 & $\sqrt{ }$ \\
\hline 94xxx & A & $\mathrm{C}$ & $\mathrm{A} R$ & A & $>90$ & 8.61 & $\mathrm{M}+\mathrm{H}^{+}$ & 476 & 476 & $\sqrt{ }$ \\
\hline $94 y y y$ & F & B & IS & B & 79 & 9.96 & $\mathrm{M}+\mathrm{Na}^{+}$ & 710 & 710 & $\sqrt{ }$ \\
\hline 94zzz & A & $\mathrm{C}$ & GS & A & 42 & 8.50 & $\mathrm{M}+\mathrm{H}^{+}$ & 458 & 458 & $\sqrt{ }$ \\
\hline 94aаaа & G & $\mathrm{C}$ & $\mathrm{FS}$ & A & 38 & 11.78 & $\mathrm{M}+\mathrm{NH}_{4}{ }^{+}$ & 865 & 865 & $\sqrt{ }$ \\
\hline 94bbbb & $\mathrm{G}$ & $\mathrm{C}$ & $\mathrm{CS}$ & B & 85 & 11.21 & $\mathrm{M}+\mathrm{NH}_{4}^{+}$ & 895 & 895 & $\sqrt{ }$ \\
\hline
\end{tabular}


Burke, Berger, and Schreiber - Supporting Information Part A

\begin{tabular}{|c|c|c|c|c|c|c|c|c|c|c|}
\hline Macrobead & $\mathbf{B B}_{1}$ & $\square_{1}$ & $\mathbf{B B}_{2}$ & $\square_{2}$ & $\begin{array}{c}\text { Purity } \\
(\%)\end{array}$ & $\begin{array}{c}\mathbf{t}_{\mathrm{R}} \\
(\mathrm{min})\end{array}$ & Ion & Calculated & Observed & $\begin{array}{l}\text { Tag-encoded } \\
\text { structure }\end{array}$ \\
\hline $94 \operatorname{cccc}$ & $\mathrm{D}$ & A & $\mathrm{O} R$ & A & 72 & 8.42 & $\mathrm{M}+\mathrm{H}^{+}$ & 486 & 486 & $\sqrt{ }$ \\
\hline 94dddd & $\mathrm{C}$ & B & $\mathrm{H} R$ & A & $>70$ & 9.04 & $\mathrm{M}+\mathrm{Na}^{+}$ & 604 & 604 & $\sqrt{ }$ \\
\hline 94eeee & $\mathrm{D}$ & $\mathrm{C}$ & $\mathrm{J} S$ & B & 80 & 8.29 & $\mathrm{M}+\mathrm{H}^{+}$ & 576 & 576 & $\sqrt{ }$ \\
\hline 94ffff & G & A & $\mathrm{N} S$ & A & 75 & 11.43 & $\mathrm{M}+\mathrm{NH}_{4}{ }^{+}$ & 835 & 835 & $\sqrt{ }$ \\
\hline 94gggg & $\mathrm{F}$ & A & $\mathrm{FS}$ & B & $>90$ & 8.30 & $\mathrm{M}+\mathrm{NH}_{4}^{+}$ & 643 & 643 & $\sqrt{ }$ \\
\hline 94hhhh & $\mathrm{E}$ & A & $\mathrm{A} S$ & B & $>90$ & 6.93 & $\mathrm{M}+\mathrm{H}^{+}$ & 534 & 534 & $\sqrt{ }$ \\
\hline 94iiii & E & $\mathrm{C}$ & $\mathrm{H} S$ & A & 66 & 8.28 & $\mathrm{M}+\mathrm{H}++$ & 566 & 566 & $\sqrt{ }$ \\
\hline 94j.j.jj & F & $\mathrm{C}$ & $\mathrm{A} S$ & A & 69 & 10.67 & $\mathrm{M}+\mathrm{H}^{+}$ & 640 & 640 & $\sqrt{ }$ \\
\hline 94kkkk & E & B & FS & A & 81 & 6.28 & $\mathrm{M}+\mathrm{NH}_{4}^{+}$ & 603 & 603 & $\sqrt{ }$ \\
\hline 941111 & $\mathrm{D}$ & B & $\mathrm{A} R$ & A & 90 & 7.00 & $\mathrm{M}+\mathrm{NH}_{4}^{+}$ & 543 & 543 & $\sqrt{ }$ \\
\hline $94 \mathrm{mmmm}$ & $\mathrm{C}$ & B & $\mathrm{J} R$ & A & $>90$ & 9.60 & $\mathrm{M}+\mathrm{Na}^{+}$ & 598 & 598 & $\sqrt{ }$ \\
\hline 94nnnn & $\mathrm{C}$ & A & $\mathrm{CS}$ & A & $>90$ & 9.71 & $\mathrm{M}+\mathrm{H}^{+}$ & 470 & 470 & $\sqrt{ }$ \\
\hline 940000 & B & $\mathrm{C}$ & $\mathrm{D} S$ & B & 73 & 9.87 & $\mathrm{M}+\mathrm{NH}_{4}^{+}$ & 595 & 595 & $\sqrt{ }$ \\
\hline 94pppp & B & A & $\mathrm{O} R$ & B & $<70$ & 9.4 & $\mathrm{M}+\mathrm{Na}^{+}$ & 566 & 566 & $\sqrt{ }$ \\
\hline 94qqqq & A & A & $\mathrm{FS}$ & A & $>90$ & 6.35 & $\mathrm{M}+\mathrm{H}^{+}$ & 416 & 416 & $\sqrt{ }$ \\
\hline 94rrrr & E & A & $\mathrm{B} S$ & A & $>90$ & 5.95 & $\mathrm{M}+\mathrm{H}^{+}$ & 426 & 426 & $\sqrt{ }$ \\
\hline 94ssss & $\mathrm{C}$ & B & $\mathrm{FS}$ & B & $>90$ & 10.90 & $\mathrm{M}+\mathrm{Na}^{+}$ & 644 & 644 & $\sqrt{ }$ \\
\hline $94 \mathrm{tttt}$ & F & $\mathrm{C}$ & $\mathrm{K} R$ & B & 79 & 10.95 & $\mathrm{M}+\mathrm{NH}_{4}{ }^{+}$ & 793 & 793 & $\sqrt{ }$ \\
\hline 94uuuu & $\mathrm{C}$ & $\mathrm{C}$ & $\mathrm{H} R$ & A & 73 & 11.05 & $\mathrm{M}+\mathrm{H}^{+}$ & 576 & 576 & $\sqrt{ }$ \\
\hline $94 \mathrm{vvvv}$ & $\mathrm{C}$ & B & $\mathrm{A} R$ & B & $>70$ & 11.58 & $\mathrm{M}+\mathrm{Na}^{+}$ & 628 & 628 & $\sqrt{ }$ \\
\hline 94wwww & F & $\mathrm{C}$ & $\mathrm{D} S$ & B & $>90$ & 10.47 & $\mathrm{M}+\mathrm{NH}_{4}^{+}$ & 717 & 717 & $\sqrt{ }$ \\
\hline $94 \times x \times x$ & $\mathrm{C}$ & B & $\mathrm{B} S$ & A & 69 & 9.62 & $\mathrm{M}+\mathrm{Na}^{+}$ & 554 & 554 & $\sqrt{ }$ \\
\hline 94yyyy & $\mathrm{F}$ & A & $\mathrm{M} R$ & B & 85 & 9.49 & $\mathrm{M}+\mathrm{NH}_{4}^{+}$ & 689 & 689 & $\sqrt{ }$ \\
\hline 94zzzz & $\mathrm{F}$ & A & $\mathrm{D} R$ & A & 69 & 8.90 & $\mathrm{M}+\mathrm{H}^{+}$ & 550 & 550 & $\sqrt{ }$ \\
\hline 94aaaaa & $\mathrm{E}$ & B & $\mathrm{L} S$ & A & 89 & 7.74 & $\mathrm{M}+\mathrm{NH}_{4}^{+}$ & 615 & 615 & $\sqrt{ }$ \\
\hline 94bbbbb & B & B & $\mathrm{H} S$ & B & $>90$ & 8.76 & $\mathrm{M}+\mathrm{Na}^{+}$ & 574 & 574 & $\sqrt{ }$ \\
\hline 94 ccecc & F & B & $\mathrm{M} S$ & B & $>90$ & 10.79 & $\mathrm{M}+\mathrm{NH}_{4}^{+}$ & 751 & 751 & $\sqrt{ }$ \\
\hline 94ddddd & $\mathrm{D}$ & A & $\mathrm{J} S$ & B & $>90$ & 6.42 & $\mathrm{M}+\mathrm{NH}_{4}^{+}$ & 503 & 503 & $\sqrt{ }$ \\
\hline 94eeeee & G & A & $\mathrm{H} R$ & B & 80 & 9.59 & $\mathrm{M}+\mathrm{NH}_{4}^{+}$ & 821 & 821 & $\sqrt{ }$ \\
\hline 94fffff & D & B & $\mathrm{N} S$ & A & $>90$ & 8.76 & $\mathrm{M}+\mathrm{Na}^{+}$ & 624 & 624 & $\sqrt{ }$ \\
\hline 94ggggg & $\mathrm{C}$ & $\mathrm{C}$ & $\mathrm{K} R$ & B & ND & $>12.5$ & $\mathrm{M}+\mathrm{Na}^{+}$ & 732 & 732 & $\sqrt{ }$ \\
\hline 94hhhhh & $\mathrm{E}$ & A & $\mathrm{F} R$ & B & $>90$ & 6.33 & $\mathrm{M}+\mathrm{NH}_{4}^{+}$ & 567 & 567 & $\sqrt{ }$ \\
\hline 94iiiiii & $\mathrm{C}$ & $\mathrm{C}$ & $\mathrm{L} R$ & A & ND & $>12.5$ & $\mathrm{M}+\mathrm{H}^{+}$ & 602 & 602 & $\sqrt{ }$ \\
\hline 94jjjjjj & D & A & $\mathrm{N} R$ & A & 65 & 8.71 & $\mathrm{M}+\mathrm{H}^{+}$ & 506 & 506 & $\sqrt{ }$ \\
\hline 94kkkkk & $\mathrm{D}$ & $\mathrm{C}$ & $\mathrm{OS}$ & A & 76 & 10.49 & $\mathrm{M}+\mathrm{H}^{+}$ & 576 & 576 & $\sqrt{ }$ \\
\hline 94IIIII & A & A & $\mathrm{E} R$ & A & $>90$ & 7.48 & $\mathrm{M}+\mathrm{H}^{+}$ & 380 & 380 & $\sqrt{ }$ \\
\hline 94mmmmm & A & $\mathrm{C}$ & $\mathrm{N} R$ & B & 35 & 11.80 & $\mathrm{M}+\mathrm{Na}^{+}$ & 648 & 648 & $\sqrt{ }$ \\
\hline 94nnnnn & $\mathrm{C}$ & $\mathrm{C}$ & I $S$ & A & $>90$ & 11.86 & $\mathrm{M}+\mathrm{H}^{+}$ & 590 & 590 & $\sqrt{ }$ \\
\hline 9400000 & $\mathrm{~F}$ & A & $\mathrm{K} R$ & A & 71 & 9.90 & $\mathrm{M}+\mathrm{H}^{+}$ & 626 & 626 & $\sqrt{ }$ \\
\hline 94ppppp & A & $\mathrm{C}$ & $\mathrm{A} R$ & A & $>90$ & 9.44 & $\mathrm{M}+\mathrm{H}^{+}$ & 490 & 490 & $\sqrt{ }$ \\
\hline
\end{tabular}

Chemical structures, HPLC traces $\left(\mathrm{UV} / \square_{214}\right.$ and $\mathrm{MS} / \mathrm{ES}^{+}$total ion current) and mass spectroscopic data $\left(\mathrm{ES}^{+}\right)$for 94a-94ppppp can be found in Supporting Information Part C. 